\title{
Produtos Caixa
}

André Ottenbreit Maschio Rodrigues

\author{
DISSERTAÇÃO APRESENTADA \\ $\mathrm{AO}$ \\ Instituto DE MATEMÁTICA E EstatísticA \\ DA \\ UNIVERSIDADE DE SÃO PAUlO \\ PARA \\ OBTENÇÃA DO TÍTULO \\ DE \\ Mestre EM Cî̂NCIAS
}

Programa: Matemática
Orientador: Profa. Dra . Lúcia Renato Junqueira

Durante o desenvolvimento deste trabalho o autor recebeu auxílio financeiro da CAPES e CNPq

São Paulo, setembro de 2014 


\section{Produtos Caixa}

Esta versão da dissertação contém as correções e alterações sugeridas pela Comissão Julgadora durante a defesa da versão original do trabalho, realizada em 12/09/2014. Uma cópia da versão original está disponível no

Instituto de Matemática e Estatística da Universidade de São Paulo.

Comissão Julgadora:

- Prof ${ }^{a}$. Dra ${ }^{a}$ Lúcia Renato Junqueira (orientadora) - IMEUSP

- Prof. Dr. Leandro Aurichi - ICMC-USP

- Prof. Dr. Samuel Gomes da Silva - UFBA 


\section{Agradecimentos}

Gostaria de agradecer primeiramente aos meus pais por todo o apoio e compreensão, sempre me incentivando e me permitindo avançar em meus estudos.

Agradeço a minha orientadora, Prof ${ }^{a}$ Lúcia Renato Junqueira, pela paciência e todo o apoio e atenção.

Agradeço a todos os meus amigos e colegas do IME-USP por me acompanharem por tantos momentos desde a graduação até o mestrado.

Agradeço aos meus companheiro de república, com quem eu compartilhei um teto e tantas lembranças durante os últimos anos.

Agradeço a todos os professores do IME-USP que me ensinaram praticamente tudo o que sei sobre Matemática, em especial à professora Iryna Kashuba pelo apoio durante a iniciação científica.

Agradeço a todos os colegas e professores do Grupo de Topologia, tanto os do IME quanto os do ICMC em São Carlos e da UFBA em Salvador.

Agradeço à CAPES e à $\mathrm{CNPq}$ pelo apoio financeiro durante a produção deste trabalho.

Agradeço também a Prof ${ }^{a}$ Ofélia Teresa Alas e Gabriel Zanetti Nunes Fernandes pelas contribuições a este trabalho. Em especial a Santi Spadaro pelo resultado do teorema 1.2.22. 


\section{Resumo}

RODRIGUES, A. O. M. Produtos Caixa. 2014. Dissertação (Mestrado) Instituto de Matemática e Estatística, Universidade de São Paulo, São Paulo, 2014.

Este trabalho tem como objetivo apresentar uma coletânea de resultados sobre produtos caixa de espaços topológicos. Com o intuito de comparação com o produto de Tychonoff, apresentamos propriedades do produto caixa relacionadas a espaços discretamente gerados e ao espaço $C_{\square}(X)$. Em seguida apresentamos alguns resultados relativos ao problema da paracompacidade de produtos caixa, o qual é a principal questão envolvendo este tipo de produto. Por fim, utilizamos o produto caixa para construir dois exemplos de pares espaços normais cujos produtos não são normais, a saber, um espaço de Dowker e e um par de espaços $\kappa$-metrizáveis.

Palavras-chave: produto caixa, paracompacidade, espaço de Dowker, espaços $C_{\square}$-discretos, pequenos cardinais. 


\section{Abstract}

RODRIGUES, A. O. M. Box Products. 2010. 120 f. Dissertação (Mestrado) - Instituto de Matemática e Estatística, Universidade de São Paulo, São Paulo, 2010.

The aim of this work is to present a selection of results about box products of topological spaces. In order to compare this product with the Tychonoff product, we present properties of the box products related to discretely generated spaces and to the space $C_{\square}(X)$. Then we present results on the problem of paracompactness of the box products, wich is the main question regarding this kind of product. At last, we use the box products to create examples of normal spaces whose products are not norma: a Dowker Space and a pair of $\kappa$-metrizable spaces.

Keywords: box product, paracompactness, Dowker space, $C_{\square}$-discrete spaces, small cardinals. 


\section{Sumário}

$\begin{array}{ll}\text { Introdução } & 1\end{array}$

1 Preliminares 5

1.1 Teoria dos Conjuntos . . . . . . . . . . . . . . . 6

1.1.1 Filtros e Ultrafiltros . . . . . . . . . . . . . . . 6

1.1 .2 Ordens . . . . . . . . . . . . . . . . 8

1.2 Topologia Geral . . . . . . . . . . . . . . . . . . 10

1.2.1 Axiomas de separação . . . . . . . . . . . . . . . . . . 11

1.2 .2 Espaços dispersos . . . . . . . . . . . . . . . . . . . . . . 13

1.2.3 Funções cardinais . . . . . . . . . . . . . . . . . . . . 15

1.2.4 Paracompacidade . . . . . . . . . . . . . . 20

1.2.5 Espaços $\kappa$-metrizáveis . . . . . . . . . . . 23

2 Comparações com o produto de Tychonoff 25

2.1 Definições . . . . . . . . . . . . . . . . . . . . . . . . . . . . . . . . . . 25

2.2 Propriedades básicas . . . . . . . . . . . . . . . . . . 27

2.3 Espaços discretamente gerados . . . . . . . . . . . . . . . . 36

2.4 O espaço de funções contínuas . . . . . . . . . . . . . . . . . . . 41

2.4.1 Cardinais mensuráveis . . . . . . . . . . . 50

3 Paracompacidade de produtos caixa $\quad 55$

3.1 O produto nabla . . . . . . . . . . . . . . 56

3.2 Uma equivalência da Hipótese do Contínuo . . . . . . . . . . . 62

3.3 Pequenos cardinais . . . . . . . . . . . . . . . . . . . . . . 69

3.4 Produtos de ordinais . . . . . . . . . . . . . . . . . . . . . 84

3.5 O caso não enumerável . . . . . . . . . . . . . . . . . . . 86

3.6 O problema dos produtos caixa nos dias de hoje . . . . . . . 89

4 Aplicações $\quad 91$

4.1 Espaço de Dowker . . . . . . . . . . . . . . . . . . . . 91

4.2 Um produto não normal de espaços $\kappa$-metrizáveis . . . . . . 100 
A Uniformidades e o produto caixa uniforme 106 A.1 Uniformidades . . . . . . . . . . . . . . . . . . . . . . . 106

A.2 Topologia induzida . . . . . . . . . . . . . . . . . . . . . 109

A.3 Produtos . . . . . . . . . . . . . . . . . . . . . 119

Referências Bibliográficas 123

$\begin{array}{ll}\text { Índice Remissivo } & 129\end{array}$ 


\section{Introdução}

Em diversas áreas da matemática, como por exemplo a álgebra, costumase utilizar o conceito de produto cartesiano como ferramenta para criar novos objetos a partir dos já construídos. Podemos citar as somas diretas e produtos diretos de anéis, módulos, corpos, espaços vetoriais, enfim, praticamente qualquer estrutura algébrica. Inclusive, tal conceito é generalizado na Teoria de Categorias pelo produto categorial.

Já na topologia, também utilizamos o produto cartesiano de maneira semelhante. Dados dois espaços topológicos, podemos equipar o produto cartesiano desses espaços com a topologia gerada pelos produtos cartesianos dos abertos das topologias em questão. Usando a mesma ideia, estender essa definição para um produto de uma quantidade finita de espaços topológicos. Tal definição satisfaz uma série de boas propriedades que permitem tal conceito ser facilmente utilizado com diversos outros conceitos da topologia. Contudo, quando trabalhamos com um produto de uma quantidade infinita de espaços topológicos, surgem algumas dificuldades.

A maneira mais natural de se estender tal conceito de produto topológico para infinitos fatores seria a de usar a mesma ideia de equipar o produto cartesiano dos espaços topológicos com a topologia gerada por todos os produtos cartesianos de abertos de cada um dos espaços em questão. Temos assim o produto caixa. Tal nome se dá pelo fato de seus abertos básicos poderem ser interpretados como "retângulos" abertos. Contudo, no caso infinito, esta topologia não satisfaz todas as mesmas propriedades que uma topologia definida do mesmo modo no caso finito satisfaria. Por isso, os topólogos passaram a usar o produto gerado pelas projeções, conhecido como produto de Tychonoff, como produto topológico usual, já que este ainda man- 
tinha todas essas propriedades. Inclusive, o produto de Tychonoff coincide com o produto categorial na categoria dos espaços topológicos.

Apesar de não possuir as mesmas qualidades que o produto d Tychonoff, o produto caixa serve como ferramenta para construir contra-exemplos. Um dos principais exemplos desta funcionalidade dos produtos caixa é o Espaço de Dowker construído por M. E. Rudin (seção 4.1).

Em termos de axiomas de separação, o produto caixa se comporta da mesma maneira que o produto de Tychonoff até a propriedade $T_{3 \frac{1}{2}}$. No entanto, a diferença entre tais produtos se evidencia quando estudamos as propriedades de cobertura. Enquanto o produto de Tychonoff preserva a compacidade (Teorema de Tychonoff), o produto caixa não é compacto (exceto pelos casos triviais). Porém, se observarmos a paracompacidade ao invés da compacidade, veremos que existem diversas condições sob as quais o produto caixa são paracompactos. Tal problema se tornou o principal foco de estudos relacionados ao produto caixa.

Inicialmente, na década 1940, Tietze propôs a pergunta:" $\square^{\omega} \mathbb{R}$ é normal?". Já na década de 1950, Arthur Stone questionou se o produto caixa enumerável de espaços metrizáveis é normal. Em [Rud72], M. E. Rudin apresentou uma resposta positiva para a pergunta de Stone. Mais precisamente, ela demonstrou que tal produto era paracompacto. Este resultado motivou o estudo da paracompacidade dos produtos caixa.

Durante as décadas de 1970 e 1980, foram obtidos diversos resultados parciais para esta questão sob diferentes hipóteses, como por exemplo a Hipótese do Contínuo e algumas hipóteses sobre pequenos cardinais. Em [Wil84], Scott Williams propõe o uso do conceito de uniformidades como ferramenta para estudar a paracompacidade dos produtos caixa. Isto levou, posteriormente, à criação do produto caixa uniforme, o qual apresenta a mesma questão relativa à sua paracompacidade.

Além de [Wil84], podemos também citar [vD80] e [Roi11] como principais referências sobre a paracompacidade de produtos caixa, reunindo os principais resultados obtidos em cada período.

O objetivo principal deste texto é apresentar um panorama sobre os principais resultados a respeito dos produtos caixa. O foco principal é o problema 
da paracompacidade, no entanto estudaremos outras propriedades deste produto a fim de compará-lo com o produto de Tychonoff, além de apresentar algumas aplicações.

A seguir detalhamos o modo como o presente trabalho está organizado.

No primeiro capítulo apresentamos conceitos de Teoria dos Conjuntos e Topologia Geral que utilizaremos ao longo do texto, além de fixar as notações utilizadas.

O segundo capítulo reúne resultados sobre o produto caixa em perspectiva com o produto de Tychonoff. Estudamos propriedades básicas como projeções, axiomas de separação, peso e caráter, conexidade, compacidade, preservação do fecho e convergência de sequências.

Em seguida, ainda no segundo capítulo exploramos alguns resultados sobre espaços discretamente gerados (propriedade introduzida por Dow, Tkachenko, Tkachuk e Wilson em [DTTW02]). Este é um exemplo de propriedade que "se comporta bem" com o produto caixa, mas o mesmo não acontece com o produto de Tychonoff, ao contrário do que acontece com diversas outras propriedades.

No final do capítulo, estudamos o espaço das funções contínuas de um espaço topológico $X$ em $\mathbb{R}$. Analogamente ao espaço $C_{p}(X)$, podemos utilizar o produto caixa para definir o espaço $C_{\square}(X)$, o qual foi introduzido por Tamariz-Mascarúa e Villegas-Rodríguez em [TV02]. Diferentemente de $C_{p}(X)$, em diversos casos o espaço $C_{\square}(X)$ pode ser discreto. Estudamos propriedades do espaço $X$ que tornam o espaço $C_{\square}(X)$ discreto. Tais propriedades estão relacionadas com o conceito de quase- $\omega$-resolvibilidade. Estudamos também um pouco de como essas propriedades se comportam em modelos de ZFC sem cardinais mensuráveis.

No terceiro capítulo apresentamos alguns resultados para o problema da paracompacidade do produto caixa. Apresentamos uma das principais ferramentas utilizadas para o estudo desta questão, o produto nabla. Tal produto nos permite estudar os produtos caixa a menos de um número finito de coordenadas. Utilizando esta ferramenta, apresentamos uma equivalência da Hipótese do Contínuo envolvendo produtos caixa e o grau de Lindelöf. Estudamos também resultados obtidos com outras hipóteses de consistência mais 
fracas, envolvendo os chamados pequenos cardinais. Apresentamos também um pouco do caso em que os fatores do produto caixa são ordinais. Por fim, mostramos um pouco dos resultados conhecidos para produtos de uma quantidade não enumerável de fatores, além de citar algumas questões ainda em aberto.

No quarto capítulo, apresentamos duas aplicações do produto caixa na questão de espaços normais cujos produtos não são normais. Primeiramente apresentamos o espaço de Dowker construído por M. E. Rudin em [Rud71]. Este consiste de um espaço normal cujo produto com o intervalo real unitário não é normal. Em seguida, apresentamos um exemplo de dois espaços $\kappa$-metrizáveis cujo produto não é normal, o qual foi construído por J. E. Vaughan em [Vau75].

Por fim, no apêndice A apresentamos as definições e alguns resultados básicos sobre uniformidades, a fim de ilustrar sua utilização como ferramenta para o produto caixa. 


\section{Capítulo 1}

\section{Preliminares}

Neste capítulo introduziremos os conceitos básicos, definições e notações que utilizaremos por todo o texto. Trabalharemos sempre assumindo os axiomas de ZFC.

Começamos introduzindo algumas notações e conceitos básicos.

Notação. Denotaremos por

- $\omega=\omega_{0}$ o conjunto $\mathbb{N}$ dos números naturais;

- $\mathbb{N}^{*}=\mathbb{N} \backslash\{0\}$ o conjunto dos números naturais não nulos;

- $\aleph_{0}$ a cardinalidade do conjunto dos números naturais $\omega$;

- $\mathbb{R}$ o conjunto dos números reais, equipado com a topologia usual;

- $\mathbb{R}_{+}^{*}$ o conjunto dos números reais positivos;

- II o intervalo unitário real $\{x \in \mathbb{R}: 0 \leq x \leq 1\}$;

- $\mathfrak{c}=2^{\aleph_{0}}=|\mathbb{R}|$ a cardinalidade do conjunto dos números reais;

- $B_{d}(x, r)=\{m \in M: d(x, m)<r\}$ a bola aberta de centro $x$ e raio $r \in \mathbb{R}_{+}$em um espaço métrico $(M, d)$;

- $B_{r}(x)=B_{d}(x, r)$, quando a métrica d estiver subentendida;

- $A^{c}$ o complementar de um conjunto $A \subset X$, ou seja, $A^{c}=X \backslash A$; 
- $\bar{C}$ o fecho de um conjunto $C$ em um espaço topológico;

- $\operatorname{dom}(f)$ o dominio de uma função $f$;

- $\mathcal{P}(X)=\{S: S \subset X\}$ o conjunto das partes de $X$;

- $[X]^{\kappa}=\{S \in \mathcal{P}(X):|S|=\kappa\}$ o conjunto das partes de tamanho $\kappa$. $[X]^{<\kappa},[X]^{\leq \kappa}$ são definidos analogamente.

\section{$1.1 \quad$ Teoria dos Conjuntos}

A menos de noção contrária, todos os resultados e definições nesta seção podem ser encontrados em [Jec03] ou [Kun11].

\subsubsection{Filtros e Ultrafiltros}

Definição 1.1.1. Seja $X$ um conjunto. Dizemos que $\mathfrak{F} \subset \mathcal{P}(X)$ é um filtro sobre $X$ se $\mathfrak{F}$ satisfaz as seguintes condições:

- $\emptyset \notin \mathfrak{F}$

- $A, B \in \mathfrak{F} \Rightarrow A \cap B \in \mathfrak{F}$

- $A \in \mathfrak{F}, A \subset B \Rightarrow B \in \mathfrak{F}$

Se, além disso, $\mathfrak{F}$ for maximal (no sentido em que, dado um filtro $\mathfrak{F}^{\prime}$ sobre $X$, se $\mathfrak{F} \subset \mathfrak{F}^{\prime}$, então $\mathfrak{F}=\mathfrak{F}^{\prime}$ ), chamamos $\mathfrak{F}$ de ultrafiltro sobre $X$.

Do mesmo modo que com os espaços topológicos, podemos definir o conceito de base de filtro.

Definição 1.1.2. Um conjunto não vazio $\mathfrak{B} \subset \mathcal{P}(X)$ é denominado base de filtro se

- $\emptyset \notin \mathfrak{B}$

- $\forall A, B \in \mathfrak{B}, \exists C \in \mathfrak{B}, C \subset A \cap B$ 
Dada uma base de filtro $\mathfrak{B}$, definimos o filtro gerado por $\mathfrak{B}$ por

$$
\mathfrak{F}=\{C \in \mathcal{P}(X): \exists B \in \mathfrak{B}, B \subset C\}
$$

Dado um filtro $\mathfrak{F}$, dizemos que $\mathfrak{B} \subset \mathfrak{F}$ é uma base para $\mathfrak{F}$ se $\mathfrak{B}$ satisfaz as condições acima e $\mathfrak{F}$ é o filtro gerado por $\mathfrak{B}$.

Definição 1.1.3. Seja $\mathfrak{F}$ um filtro sobre $X$, dizemos que $\mathfrak{F}$ é um filtro principal se $\mathfrak{F}$ é existe algum conjunto $C \subset X$ tal que $\mathfrak{F}$ é gerado por $\{C\}$. Caso contrário, dizemos que $\mathfrak{F}$ é um filtro livre.

Um dos principais conceitos relacionados aos filtros são o ultrafiltros.

Definição 1.1.4. Seja $X$ um conjunto, $\mathfrak{F}$ um filtro sobre $X$ e $\kappa$ um cardinal infinito. Dizemos que $\mathfrak{F}$ é um filtro $\kappa$-completo se qualquer intersecção de menos que $\kappa$ elementos de $\mathfrak{F}$ é um elemento de $\mathfrak{F}$.

Proposição 1.1.5. Seja $\mathfrak{F}$ um filtro sobre $X$. Então $\mathfrak{F}$ é um ultrafiltro sobre $X$ se e somente se para todo subconjunto $A \subset X$ temos ou $A \in U$ ou $X \backslash A \in$ $U$.

Demonstração. Por um lado, suponha que para todo $A \subset X$ vale que ou $A$ ou seu complementar pertence a $\mathfrak{F}$. Seja $\mathfrak{F}^{\prime}$ um filtro sobre $X$ tal que $\mathfrak{F} \subset \mathfrak{F}^{\prime}$, e suponha que exista $C \in \mathfrak{F}^{\prime}$ tal que $C \notin \mathfrak{F}$. Então temos que $X \backslash C \in \mathfrak{F}$, e portanto $X \backslash C \in \mathfrak{F}^{\prime}$, o que contradiz o fato de que $\mathfrak{F}^{\prime}$ é um filtro. Portanto, $\mathfrak{F}$ é um ultrafiltro.

Por outro lado, suponha que $\mathfrak{F}$ é um ultrafiltro. Seja $A \subset X$ tal que $X \backslash A \notin \mathfrak{F}$. Note que o conjunto $\mathfrak{B}=\mathfrak{F} \cup\{A\}$ é uma base de filtro. De fato, dado $C \in \mathcal{F}$ temos $C \cap A=\emptyset$, pois caso contrário teríamos $C \subset X \backslash A$ e consequentemente $X \backslash A \in \mathfrak{F}$. Considere então $\mathfrak{F}^{\prime}$ o filtro gerado por $\mathfrak{B}$. Temos que $A \in \mathfrak{F}^{\prime}$ e que $\mathfrak{F} \subset \mathfrak{F}^{\prime}$. Como $\mathfrak{F}$ é um ultrafiltro, temos que $\mathfrak{F}=\mathfrak{F}^{\prime}$ e , portanto, $A \in \mathfrak{F}^{\prime}$, como gostaríamos. 


\subsubsection{Ordens}

Ainda referente a definições básicas e notações, trataremos nesta seção de conceitos referentes a ordens.

Definição 1.1.6. Uma ordem ${ }^{1}$ em um conjunto $X$ é uma relação binária reflexiva, anti-simétrica e transitiva. Se, além disso, todo par de elementos do conjunto for comparável pela ordem, então chamaremos tal ordem de ordem linear. Por fim, se todo subconjunto não vazio de $X$ possuir minimo, então temos uma boa ordem.

Notação. Seja X um conjunto equipado de uma ordem $\leq$. Denotaremos por $<$ a ordem estrita induzida por $\leq$, ou seja, < é a relação definida binária por $a<b \Leftrightarrow a \leq b$ e $a \neq b$.

Denotaremos intervalos abertos e fechados usando colchetes, como a seguir:

- $[a, b]=\{x \in X: a \leq x \leq b\}$

- $] a, b[=\{x \in X: a<x<b\}$

- $]-\infty, b]=\{x \in X: x \leq b\}$

- $] a, \infty[=\{x \in X: a<x\}$

Definição 1.1.7. Dado um conjunto $\alpha$, dizemos que $\alpha$ é um ordinal se $\alpha$ é transitivo (isto é, $\forall x \in \alpha, x \subset \alpha$ ) e bem ordenado pela relação de pertinência $\in$. Dados dois cardinais $\alpha$ e $\beta$, dizemos que $\alpha<\beta$ se e somente se $\alpha \in \beta$. Dado um ordinal $\kappa$, dizemos que $\kappa$ é um cardinal se para todo ordinal $\alpha<\kappa$ não existe uma bijeção entre $\alpha$ e $\kappa$.

Quando nos referirmos a propriedades topológicas de ordinais, os consideramos com a topologia da ordem.

Definição 1.1.8. Seja $X$ um conjunto munido de uma ordem linear $\leq$. Chamamos de topologia da ordem a topologia $\tau_{\leq}$sobre o conjunto $X$ gerada pela base

$$
\{] a, \infty[: a \in X\} \cup\{]-\infty, b[: b \in X\}
$$

\footnotetext{
${ }^{1}$ conhecido também por ordem parcial
} 
Além dos conjuntos bem ordenados, utilizaremos também o conceito de árvore.

Definição 1.1.9 (Árvores).

- Um conjunto parcialmente ordenado $(T, \leq)$ é chamado árvore se, para todo $t \in T$, o conjunto $t^{\downarrow}=\{y \in T: y \leq t\}$ é bem ordenado.

- Dado $t \in T$, definimos a altura de $t$ por $\mathrm{ht}(t)=\alpha$, onde $\alpha$ é o único ordinal tal que $t^{\downarrow}$ possui tipo de ordem $\alpha$, isto é, existe uma bijeção crescente entre $t^{\downarrow} e \alpha$.

- Definimos a a altura de $T$ por $\operatorname{ht}(T)=\min \alpha: \forall t \in T$, ht $(t) \neq \alpha$.

- Dado a um ordinal, definimos o $\alpha$-ésimo nível de $T$ como o conjunto $\{t \in T: \operatorname{ht}(t)=\alpha\}$.

- Um ramo de uma árvore $(T, \leq)$ é uma cadeia maximal em $T$, isto é, subconjunto $C \subset T$ tal que todo par de elementos de $T$ é comparável $e$ para qualquer $t \in T \backslash C$ existe um elemento $t^{\prime} \in C$ tal que $t$ e $t^{\prime}$ não são comparáveis.

Utilizaremos um resultado básico de combinatória no caso particular de árvores. Sua demonstração pode ser encontrada por exemplo em [Lév02].

Teorema 1.1.10 (Lema de König para árvores). Seja $(T, \leq)$ uma árvore de altura $\omega$ tal que cada nível de $T$ é finito. Então $T$ possui um ramo infinito.

Demonstração. Para cada $t \in T$, denotaremos o conjunto $\{p \in T: p \geq t\}$ por $t^{\uparrow}$. Vamos construir um ramo $\left\{a_{n}: n \in \omega\right\} \subset T$ por indução em $n$, tal que para cada $n \in \omega$ temos ht $\left(a_{n}\right)=n$ e $\left|a_{n}{ }^{\uparrow}\right| \geq \aleph_{0}$. Primeiramente, note que podemos escrever $T=\bigcup_{\mathrm{ht}(t)=0} t^{\uparrow}$. Como $T$ é infinito, temos que existe algum $t \in T$, com ht $(t)=0$ tal que $t^{\uparrow}$ é infinito. Definimos $a_{0}$ como tal $t$.

Suponhamos agora $a_{n}$ construído para todo $n \leq k$. Podemos agora escrever

$$
a_{n}^{\uparrow}=\left\{a_{n}\right\} \cup\left(\bigcup\left\{t^{\uparrow}: \operatorname{ht}(t)=n+1 \text { e } a_{n} \leq t\right\}\right)
$$


Pela hipótese de indução, temos $a_{n}{ }^{\uparrow}$ infinito. Portanto, podemos encontrar $t^{\prime} \geq a_{n}$, com $h t\left(t^{\prime}\right)=n+1$ tal que $t^{\prime \uparrow}$ é infinito. Definimos assim $a_{n+1}=t^{\prime}$ e completamos a indução.

Para definições e resultados sobre ordinais e cardinais, consulte [HJ99]. A menos de menção contrária, quando o texto se referir a ordinais como espaço topológico está implícito se tratar da topologia da ordem. Outro conceito importante sobre ordem a ser utilizado é o de cofinalidade.

Definição 1.1.11. Seja $X$ um conjunto equipado com uma ordem $\leq$. Dizemos que um conjunto $B \subset A$ é cofinal em $A$ se $\forall a \in A, \exists b \in B, a \leq b$

Definição 1.1.12. Dado um conjunto $C$, definimos a cofinalidade de $C$ por:

$$
\operatorname{cf}(C)=\min \{|B|: B \subset C \text { é cofinal em } C\}
$$

Definição 1.1.13. Um cardinal $\kappa$ é dito regular se $\operatorname{cf}(\kappa)=\kappa$. Se $\kappa$ não é regular, dizemos que $\kappa$ é singular.

Além de $\aleph_{0}$, qualquer cardinal sucessor (isto é, da forma $\aleph_{\alpha+1}$ para algum ordinal $\alpha$ ) é regular.

\subsection{Topologia Geral}

A menos de noção contrária, todos os resultados e definições nesta seção podem ser encontrados em [Eng89] ou [Kel75].

Definição 1.2.1. Seja $C$ um subconjunto de um espaço topológico $X$. Dizemos que $C$ é

- $G_{\delta}$ se pode ser escrito como interseção enumerável de conjuntos abertos.

- $F_{\sigma}$ se pode ser escrito como união enumerável de conjuntos fechados. 


\subsubsection{Axiomas de separação}

Definiremos nesta seção os axiomas de separação $T_{0}, T_{1}, T_{2}, T_{3}, T_{3 \frac{1}{2}}, T_{4}$. Em todos os casos, teremos $X$ um espaço topológico.

Definição 1.2.2. Dizemos que $X$ satisfaz o axioma $T_{0}$ ou que $X$ é um espaço $T_{0}$ se para cada par não ordenado de pontos de $X$ existe um aberto que contém um ponto e não contém o outro.

Definição 1.2.3. Dizemos que $X$ satisfaz o axioma $T_{1}$ ou que $X$ é um espaço $T_{1}$ se, dados $x, y \in X$ distintos, existe uma vizinhança de $x$ que não contém $y$.

Definição 1.2.4. Dizemos que $X$ satisfaz o axioma $T_{2}$ ou que $X$ é um espaço $T_{2}$ se, dados $x, y \in X$ distintos, existem abertos $U, V$ disjuntos tais que $x \in U$ e $y \in V$. Também chamamos um espaço $T_{2}$ de espaço Hausdorff

Definição 1.2.5. Dizemos que $X$ satisfaz o axioma $T_{3}$ ou que $X$ é um espaço $T_{3}$ se, dado um fechado $F \subset X$ e um ponto $x \notin F$, temos abertos $U, V \subset X$ disjuntos tais que $x \in U$ e $F \subset V$. Se $X$ é um espaço $T_{1}$ e $T_{3}$, chamamos $X$ de espaço regular.

Proposição 1.2.6. Dado X um espaço topológico, são equivalentes:

1. $X$ é $T_{3}$;

2. para qualquer $x \in X$ e qualquer vizinhança aberta $W$ de $x$, existe um aberto $U$ tal que $x \in U \subset \bar{U} \subset W$.

Demonstração. Suponha $X$ um espaço topológico $T_{3}$. Fixemos $x \in X$ e uma vizinhança aberta $W$ de $x$. Note que o conjunto $F=X \backslash W$ é um fechado ao qual $x$ não pertence. Logo, como $X$ é $T_{3}$, existem abertos $U$ e $V$ tais que $x \in U, F \subset V$ e $U \cap V=\emptyset$. Note que $\bar{U} \cap F=\emptyset$, pois dado $p \in F$, temos que $V$ é uma vizinhança de $p$ que não intersecciona $U$. Assim, temos $x \in U \subset \bar{U} \subset W$, de modo que $X$ satisfaz a condição 2 .

Para a recíproca, suponhamos que $X$ satisfaça a condição 2. Seja $F \subset X$ um fechado, e seja $x \in X \backslash F$. Por hipótese, existe um aberto $U$ tal que $x \in U \subset U \subset \bar{U} \subset X \backslash F$. Temos assim os abertos $U$ e $V=X \backslash \bar{U}$ tais que $x \in U, F \subset V$ e $U \cap V=\emptyset$ e, portanto, $X$ é $T_{3}$ como queríamos. 
Utilizaremos uma aplicação da regularidade que envolve a seguinte definição.

\section{Definição 1.2.7.}

- Um espaço topológico com a propriedade de que a interseção enumerável de abertos é aberto é chamado de $\mathrm{P}$-espaço.

- Um espaço topológico é dito zero dimensional se possui uma base formada por abertos-fechados.

Proposição 1.2.8. Se X é um P-espaço satisfazendo o axioma $T_{3}$, então $X$ é zero-dimensional.

Demonstração. Dados $x \in X$ e $U \subset X$ aberto tal que $x \in U$, como $X$ é $T_{3}$, existe aberto $V_{0}$ tal que $x \in V_{0} \subset \overline{V_{0}} \subset U$. Podemos então construir uma sequência de abertos $\left(V_{n}\right)_{n \in \omega}$ tal que para cada $n \in \omega$ temos $x \in V_{n+1} \subset \overline{V_{n+1}} \subset V_{n}$. Assim, temos $\bigcap_{n \in \omega} V_{n}=\bigcap_{n \in \omega} \overline{V_{n}}$. Tal conjunto é um aberto-fechado, pois $X$ é P-espaço. Assim, construímos uma base de abertos-fechados para $X$.

Definição 1.2.9. Dizemos que $X$ satisfaz o axioma $T_{3 \frac{1}{2}}$ ou que $X$ é um espaço $T_{3 \frac{1}{2}}$ se, dado um fechado $F \subset X$ e um ponto $x \notin F$, existe uma função contínua $f: X \longrightarrow \mathbb{I}$ tal que $f(x)=0$ e $f(y)=1$ para qualquer $y \in F$. Se $X$ é simultaneamente $T_{1}$ e $T_{3 \frac{1}{2}}$, dizemos que $X$ é de Tychonoff ou completamente regular.

Definição 1.2.10. Dizemos que $X$ satisfaz o axioma $T_{4}$ ou que $X$ é um espaço $T_{4}$ se, dados dois fechados disjuntos $F_{1}$ e $F_{2}$, existem dois abertos disjuntos $U, V$ tais que $F_{1} \subset U$ e $F_{2} \subset V$. Se $X$ é um espaço $T_{1}$ e $T_{4}$, chamamos $X$ de espaço normal.

Ao longo do texto, utilizaremos dois tipos mais fortes de normalidade: a coletiva e a monótona.

Definição 1.2.11. Sejam $X$ um espaço topológico e $\mathcal{A} \subset \mathcal{P}(X)$ uma família de subconjuntos de $X$. Dizemos que: 
- $\mathcal{A}$ é uma familia discreta se todo ponto $x \in X$ possui uma vizinhança $V_{x}$ que intercepta no máximo 1 elemento de $\mathcal{A}$.

- X é coletivamente normal se, dada uma família discreta de fechados $\left\{F_{i} \subset X: i \in I\right\}$, existe uma familia de abertos dois a dois disjuntos $\left\{A_{i} \subset X: i \in I\right\}$ tal que $\forall i \in I, F_{i} \subset A_{i}$.

Definição 1.2.12. Um espaço topológico $T_{1}(X, \tau)$ é dito monotonamente normal se para todo par $(x, A)$, com $x \in A \in \tau$, existe um aberto $\mu(x, A)$ satisfazendo:

1. $x \in \mu(x, A) \subset A$

2. se $\mu(x, A) \cap \mu(y, B) \neq \emptyset$, então $x \in B$ ou $y \in A$

Diferentemente da normalidade, a propriedade 1.2.12 é hereditária, o que é facilmente verificável graças a sua caracterização essencialmente local.

Para justificar o nome desta propriedade, note que todo espaço monotonamente normal é, em particular, normal: dados dois fechados disjuntos $F, G \subset X$, estes são separados pelos abertos $U=\bigcup_{p \in F} \mu(p, X \backslash G)$ e $V=\bigcup_{q \in G} \mu(q, X \backslash F)$, respectivamente. A contrapositiva do item 2 da definição garante que $U$ e $V$ são disjuntos. Além disso, se "aumentarmos" o fechado $F$ e "diminuirmos" $G$, os abertos $U$ e $V$ também "crescem" e "diminuem" (respectivamente) monotonamente. Isto é, dados fechados $F^{\prime} \supset F$ e $G^{\prime} \subset G$, podemos de maneira análoga construir a partir de $\mu$ abertos disjuntos $U^{\prime}$ e $V^{\prime}$ tais que $U \subset U^{\prime}, V^{\prime} \subset V, F^{\prime} \subset U^{\prime}$ e $G^{\prime} \subset V^{\prime}$.

\subsubsection{Espaços dispersos}

Uma propriedade topológica dos conjuntos bem ordenados é que os mesmos são dispersos. Por esta razão, utilizaremos o conceito de espaço disperso para estudar o produto caixa de ordinais na seção 3.4.

Definição 1.2.13. Seja $X$ um espaço topológico. Dizemos que $X$ é disperso se para todo $A \subset X, A \neq \emptyset$, existe um ponto isolado no subespaço $A$. 
Note que todo conjunto bem ordenado equipado com a topologia da ordem é disperso. De fato, dado $X$ conjunto bem ordenado e dado $Y \subset X$, temos que $p=\min Y$ é ponto isolado de $Y$, visto que existe o intervalo aberto ] $0, p+1[$ de extremos $0=\min X$ e o sucessor de $p$. Em particular, temos que todo ordinal é disperso.

Podemos caracterizar a dispersão de um espaço topológico pela sequência de Cantor-Bendixon. Dado um espaço topológico $X$ qualquer, podemos definir a seguinte sequência de subespaços: Começamos com $X^{(0)}=$ $X$. Supondo $X^{(\alpha)}$ construído para algum ordinal $\alpha$, definimos $X^{(\alpha+1)}=$ $\left\{x \in X^{(\alpha)}: x\right.$ não é isolado em $\left.X^{(\alpha)}\right\}$. Se $\theta$ é um ordinal limite, definimos $X^{(\theta)}=\bigcap_{\alpha<\theta} X^{(\alpha)}$. Por indução, dado qualquer ordinal $\alpha$ podemos ver que $X^{(\alpha)}$ é fechado em $X$ e que dado $Y \subset X$ temos $Y^{(\alpha)} \subset X^{(\alpha)}$.

Proposição 1.2.14. Um espaço topológico $X$ é disperso se e somente se existe algum ordinal $\alpha$ tal que $X^{(\alpha)}=\emptyset$.

Demonstração. Suponha $X$ disperso. Para qualquer ordinal $\alpha$ temos $X^{(\alpha)} \subset$ $X$. Portanto, se $X^{(\alpha)} \neq \emptyset$, então o mesmo tem um ponto isolado, de maneira que $\alpha X^{(\alpha+1)} \varsubsetneqq X^{(\alpha)}$. Assim, a cada iteração da sequência de CantorBendixon, retira-se algum ponto, de maneira que $X^{(\kappa)}=\emptyset$ para $\kappa>|X|^{+}$.

Suponhamos agora que exista um ordinal $\alpha$ tal que $X^{(\alpha)}=\emptyset$. Dado $Y \subset X$ não vazio, temos que $Y^{(\alpha)} \subset X^{(\alpha)}=\emptyset$. Disso, concluímos que $Y$ possuí algum ponto isolado pois, caso o contrário, teríamos $Y=Y^{(0)}=$ $Y^{(1)}=\ldots=Y^{(\alpha)}$, o que é um absurdo.

Observação 1.2.15. Se além de disperso, $X$ for também compacto, temos que o menor ordinal $\alpha$ é tal que $X^{(\alpha)}=\emptyset$ é um ordinal sucessor , isto é, $\alpha=\beta+1$. Além disso, temos que $X^{(\beta)}$ é finito. De fato, se $\alpha$ é ordinal limite, ou se $X^{(\beta)}$ fosse infinito, teríamos uma quantidade infinita de pontos isolados em algum subespaço fechado de $X$, o que é impossivel. Denominamos tal $\beta$ por rank de $X$, e denotamos por $\beta=\operatorname{rank}(X)$. 


\subsubsection{Funções cardinais}

Chamamos de função cardinal uma "função" que a associa a cada espaço topológico um cardinal, de maneira que espaços homeomorfos são levados ao mesmo cardinal. Tecnicamente, uma função cardinal não pode ser uma função no sentido de teoria dos conjuntos, pois o seu domínio, caso existisse, seria equipotente ao conjunto de todos os conjuntos, o qual não existe. Por essa razão, trabalharemos com funções cardinais apenas como notação.

Seguem alguns exemplos clássicos de funções cardinais, os quais serão usados neste texto para facilitar a notação. Mais informações sobre essas funções podem ser encontradas em [Hod84].

Definição 1.2.16. Seja $X$ um espaço topológico. Denominamos por grau de Lindelöf de $X$ (e denotamos $L(X)$ ) o menor cardinal $\kappa$ tal que toda cobertura aberta de $X$ possui uma subcobertura de tamanho $\kappa$.

Definição 1.2.17. Seja $X$ um espaço topológico. Definimos o peso de $X$ por

$$
w(X)=\min \{|\mathcal{B}|: \mathcal{B} \text { é base de } X\}
$$

Se $w(X)=\aleph_{0}$, dizemos que $X$ satisfaz o segundo axioma da enumerabilidade, ou também que $X$ é segundo enumerável.

Definição 1.2.18. Seja $X$ um espaço topológico. Definimos a densidade de $X$ por

$$
d(X)=\min \{|D|: D \text { é denso em } X\}
$$

Se $d(X)=\aleph_{0}$, dizemos que $X$ satisfaz o terceiro axioma da enumerabilidade, ou também que $X$ é separável.

Definição 1.2.19. Seja $X$ um espaço topológico, e $C \subset \mathcal{P}(X)$. Dizemos que $C$ é uma família celular se é composta por abertos dois a dois disjuntos. Definimos então $c(X)=\sup \{|C|: C \subset \mathcal{P}(X)$ é uma família celular $\}$ como a celularidade de $X$. Se $c(x)=\aleph_{0}$, dizemos que $X$ satisfaz a condição de cadeia enumerável $(\mathrm{ccc})^{2}$.

\footnotetext{
${ }^{2}$ Do inglês, contable chain condition
} 
Definição 1.2.20. Seja $X$ um espaço topológico, e seja $x \in X$. Definimos o caráter do ponto $x$ por $\chi(x, X)=\min \left\{\left|\mathcal{B}_{x}\right|: \mathcal{B}_{x}\right.$ é uma base local para $\left.x\right\}$. Definimos então o caráter do espaço $X$ por $\chi(X)=\sup _{x \in X} \chi(x, X)$. Se $\chi(X)=\aleph_{0}$, dizemos que $X$ satisfaz o primeiro axioma da enumerabilidade, ou também que $X$ é primeiro enumerável.

O caráter é o exemplo de uma função cardinal local, isto é, uma função que é construída a partir de uma função definida em cada ponto do espaço.

Com o objetivo de majorar a cardinalidade de espaços Hausdorff, utilizaremos o seguinte conhecido resultado de Arhangel'skii.

Teorema 1.2.21 (Arhangelskii). Seja X um espaço Hausdorff. Então

$$
|X| \leq 2^{L(X) \chi(X)}
$$

Demonstração. Ao invés da demonstração original, utilizaremos a versão de R. Pol [Pol74].

Fixemos $\kappa=L(X) \chi(X)$. Para cada $x \in X$, fixemos $\mathcal{V}_{x}=\left\{V_{x, \lambda}: \lambda<\kappa\right\}$ uma base local para $x$. Vamos construir indutivamente uma sequência crescente $\left(F_{\alpha}\right)_{\alpha<\kappa^{+}}$de fechados em $X$ satisfazendo:

(1) $\forall \alpha<\kappa^{+},\left|F_{\alpha}\right| \leq 2^{\kappa}$;

(2) para $0<\alpha<\kappa^{+}$, dado qualquer $\mathcal{U} \in\left[\bigcup\left\{\mathcal{V}_{x}: x \in \bigcup_{\beta<\alpha} F_{\beta}\right\}\right]^{\leq \kappa}$, temos

$$
X \backslash \bigcup \mathcal{U} \neq \emptyset \Longrightarrow F_{\alpha} \backslash \mathcal{U} \neq \emptyset
$$

Começamos definindo $F_{0}=\{p\}$ para algum $p \in X$ qualquer. Fixado $\alpha<\kappa^{+}$, suponhamos $F_{\beta}$ construído para cada $\beta<\alpha$. Definimos então

$$
\mathcal{V}=\bigcup\left\{\mathcal{V}_{x}: x \in \bigcup_{\beta<\alpha} F_{\beta}\right\} \text { e } \mathcal{V}^{*}=\left\{X \backslash \bigcup \mathcal{U}: \mathcal{U} \in[\mathcal{V}]^{\leq \kappa}\right\}
$$

Como $|\mathcal{V}| \leq 2^{\kappa}$, temos $\left|\mathcal{V}^{*}\right| \leq=\left(2^{\kappa}\right)^{\kappa}=2^{\kappa}$. Escolhemos então um ponto 
de cada elemento de $\mathcal{V}^{*}$ e obtemos um conjunto $E$ tal que $|E| \leq 2^{\kappa}$. Definimos

$$
F_{\alpha}=\overline{E \cup \bigcup_{\beta<\alpha} F_{\beta}} .
$$

Por construção, temos que $F_{\alpha}$ satisfaz (2). Vamos mostrar que $F_{\alpha}$ satisfaz (1). Para cada $x \in F_{\alpha}$ e cada $\lambda<\kappa$, escolhemos

$$
a_{x, \lambda} \in V_{x, \lambda} \cap\left(E \cup \bigcup_{\beta<\alpha} F_{\beta}\right)
$$

qualquer. Note que a sequência $\left(a_{x, \lambda}\right)_{\lambda<\kappa}$ converge para $x$. Definimos $\Phi$ : $F_{\alpha} \longrightarrow\left(E \cup \bigcup_{\beta<\alpha} F_{\beta}\right)^{\kappa}$ a função que associa cada ponto $x \in F_{\alpha}$ à sequência $\left(a_{x, \lambda}\right)_{\lambda<\kappa}$ acima construída. Como $X$ é Hausdorff, temos que cada sequência de pontos de $X$ converge para um único ponto, de modo que $\Phi$ é injetora. Portanto, $\left|F_{\alpha}\right| \leq\left(2^{\kappa}\right)^{\kappa}$, como gostaríamos.

Uma vez construída a sequência $\left(F_{\alpha}\right)_{\alpha<\kappa^{+}}$, definimos $F=\bigcup_{\alpha<\kappa^{+}} F_{\alpha}$. Vamos mostrar que $X=F$. Primeiramente, vamos mostrar que $F$ é fechado. Seja $a \in \bar{F}$. Para cada $\lambda<\kappa$, fixemos um ponto $a_{\lambda} \in V_{a, \lambda} \cap F$ qualquer. Note que a sequência $\left(a_{\lambda}\right)_{\lambda<\kappa}$ converge para $a$. Como $\left(F_{\alpha}\right)_{\alpha<\kappa^{+}}$é crescente e $\kappa^{+}$é um cardinal regular, podemos encontrar $\alpha<\kappa^{+}$tal que $\left\{a_{\lambda}: \lambda<\kappa\right\} \subset F_{\alpha}$. Logo $a \in \overline{F_{\alpha}}=F_{\alpha} \subset F$.

Suponhamos agora, por absurdo, que exista $y \in X \backslash F$. Para cada $x \in F$, podemos encontrar $V_{x} \in \mathcal{V}_{x}$ tal que $y \notin V_{x}$. Temos que a família $\left\{V_{x}: x \in F\right\}$ cobre $F$. Como $F$ é fechado, temos que $L(F) \leq \kappa$, e portanto, podemos encontrar $F^{\prime} \in[F]^{\leq \kappa}$ tal que a família $\mathcal{U}=\left\{V_{x}: x \in F^{\prime}\right\}$ também cobre $F$. Novamente pelo fato de que $\kappa$ é regular, temos que existe algum $\gamma<\kappa^{+}$tal que $F^{\prime} \subset F_{\gamma}$. Note que $\mathcal{U} \in\left[\bigcup\left\{\mathcal{V}_{x}: x \in \bigcup_{\beta<\gamma} F_{\beta}\right\}\right]^{\leq \kappa} \mathrm{e}$ $y \in X \backslash \bigcup \mathcal{U}$. Por 2 , temos que $F_{\gamma} \backslash \bigcup \mathcal{U} \neq \emptyset$, o que contradiz o fato de que $\mathcal{U}$ cobre $F$. Logo, $X=F$, como queríamos.

Do mesmo modo que o teorema anterior pode ser também demonstrado utilizando técnicas de submodelos elementares, utilizaremos tais técnicas para demonstrar o próximo teorema. Trata-se de um resultado de Arhangel'skii não publicado, porém citado por Kunen em [Kun78], o qual generaliza um 
caso específico do teorema 1.2.21. A presente demonstração foi formulada por Santi Spadaro [hs].

Teorema 1.2.22. Sejam $(X, \tau)$ um espaço compacto Hausdorff e $\mathcal{F}$ uma cobertura de $X$ por fechados $G_{\delta}$. Se $\mathcal{F}$ satisfaz

$$
\forall U \in \mathcal{F},|\{V \in \mathcal{F}: U \cap V \neq \emptyset\}| \leq \mathfrak{c}
$$

então

$$
|\mathcal{F}| \leq \mathfrak{c}
$$

Demonstração. Para esta demonstração, utilizaremos técnicas básicas de submodelos elementares. Para detalhes sobre submodelos elementares, sugerimos [Dow88] e [Dia08].

Seja $\theta$ um cardinal regular suficientemente grande, e $M$ um submodelo elementar de $H(\theta)$ enumeravelmente fechado (isto é, $M^{\omega} \subset M$ ) tal que $|M|=$ $\mathfrak{c}, \mathfrak{c} \subset M$ e $\{X, \tau, \mathcal{F}, \mathfrak{c}\} \in M$. O teorema de Löwenheim-Skolem garante a existência de tal $M$. $M$ tem a seguinte propriedade:

$$
A \in M \text { e }|A| \leq \mathfrak{c} \Longrightarrow A \subset M
$$

Nosso objetivo é mostrar que $\mathcal{F} \subset M$, do que se conclui que $|\mathcal{F}| \leq \mathfrak{c}$, como gostaríamos.

Como $X$ é compacto Hausdorff, temos que $X$ é normal. Pela normalidade de $X$, para cada $F \in \mathcal{F}$ existe uma sequência decrescente de abertos $\left(V_{n}(F)\right)_{n \in \omega}$ tal que $\overline{V_{n+1}} \subset V_{n}$ para cada $n \in \omega$ e $F=\bigcap_{n \in \omega} V_{n}(F)$, uma vez que $F$ é $G_{\delta}$. Se $F \in \mathcal{F} \cap M$, podemos supor sem perda de generalidade que $\left\{V_{n}(F): n \in \omega\right\} \in M$. Pela propriedade de $M$ destacada anteriormente, temos que $\left\{V_{n}(F): n \in \omega\right\} \subset M$.

Note que para todo $x \in \overline{X \cap M}$ podemos encontrar $G \in \mathcal{F} \cap M$ tal que $x \in G$. De fato, fixemos $x \in \overline{X \cap M}$ e $G \in \mathcal{F}$ tal que $x \in G$. Vamos mostrar que $G \in M$.

Para cada $n \in \omega$, consideremos um ponto $x_{n} \in V_{n}(G) \cap X \cap M$ qualquer. Note que a sequência $\left(x_{n}\right)_{n \in \omega}$ converge para $G$, no sentido que para qualquer 
aberto $U \supset G$ existe $n_{0} \in \omega$ tal que para todo $n>n_{0}$ temos $x_{n} \in U$. De fato, dado um aberto $U \supset G$, temos que $X \backslash U$ é compacto, logo existe uma subcobertura finita de $\left\{V_{n}(G): n \in \omega\right\}$ sobre $X \backslash U$. Assim, existe $n_{0} \in \omega$ tal que $V_{n_{0}}(G) \subset U$, de modo que $x_{n} \in U$ para todo $n>n_{0}$.

Como $M$ é enumeravelmente fechado, temos que para cada $n \in \omega$ temos $\left\{x_{i}: i \geq n\right\} \in M$. Assim, temos que o conjunto

$$
S=\left\{F \in \mathcal{F}:\left(x_{n}\right)_{n \in \omega} \text { converge para } F\right\}
$$

é um elemento de $M$, pois todas as variáveis livres da fórmula que define $S$ pertencem a $M$.

Note que, como $X$ é normal, dado qualquer $F \in S$, temos $G \cap F \neq \emptyset$. De fato, se isso não acontecesse, poderíamos separar $G$ e $F$ por abertos disjuntos, o que contradiria o fato de que $\left(x_{n}\right)_{n \in \omega}$ converge para $G$ e para $F$. Assim, pela propriedade que $\mathcal{F}$, temos que $|S| \leq \mathfrak{c}$. Portanto temos $S \subset M$, $\log$ o $G \in M$, como gostaríamos.

Para cada $F \in \mathcal{F}$ temos que $\{H \in \mathcal{F}: H \cap F \neq \emptyset\} \in M$, pois todas as variáveis livres da fórmula que define tal conjunto estão em $M$. Temos então que $\{H \in \mathcal{F}: H \cap F \neq \emptyset\} \subset M$, pois por hipótese $G$ intersecciona no máximo $\mathfrak{c}$ elementos de $\mathcal{F}$.

Vamos agora supor por absurdo que $\mathcal{F} \not \subset M$. Seja então $K \in \mathcal{F} \backslash M$. Temos que $K$ não intersecciona nenhum elemento de $\mathcal{F}$. Logo, para cada $F \in \mathcal{F}$ podemos encontrar $m \in \omega$ tal que $V_{m}(F) \cap K=\emptyset$, pois a cobertura $\{X \backslash K\} \cup\left\{X \backslash V_{n}(F): n \in \omega\right\}$ de $X$ tem subcobertura finita.

Assim, para cada $x \in \overline{X \cap M}$, podemos encontrar uma vizinhança $U_{x} \in$ $M$ de $x$ tal que $U_{x} \cap K=\emptyset$.

Considere agora $\mathcal{U}=\left\{U_{x}: x \in \overline{X \cap M}\right\}$. Note que $\mathcal{U} \subset M$ e que, como $\overline{X \cap M}$ é compacto, existe $\mathcal{U}^{\prime} \in[\mathcal{U}]^{<\aleph_{0}}$ tal que $\overline{X \cap M} \subset \mathcal{U U}^{\prime}$. Como todas as variáveis livres em questão estão em $M$, temos que

$$
M \models X \subset \bigcup \mathcal{U}^{\prime}
$$


e como $M$ é submodelo elementar de $H(\theta)$, temos que

$$
H(\theta) \models X \subset \bigcup \mathcal{U}^{\prime}
$$

No entanto, isso contradiz $K \cap \bigcup \mathcal{U}^{\prime}=\emptyset$. Logo, $\mathcal{F} \subset M$, como queríamos.

\subsubsection{Paracompacidade}

Definição 1.2.23. Sejam $X$ um espaço topológico, $\mathcal{U}, \mathcal{V} \subset \mathcal{P}(X)$ famílias de subconjuntos de $X$. Dizemos que:

- $\mathcal{U}$ é localmente finita se para todo $x \in X$ existe uma vizinhança $U_{x}$ de $x$ a qual é interceptada por no máximo uma quantidade finita de elementos de $\mathcal{U}$ (ou seja, o conjunto $\left\{U \in \mathcal{U}: U \cap U_{x} \neq \emptyset\right\}$ é finito)

- $\mathcal{U}$ é $\sigma$-localmente finita se puder ser escrita como união enumerável de famílias localmente finitas.

- $\mathcal{V}$ refina $\mathcal{U}$ se $\bigcup \mathcal{V}=\bigcup \mathcal{U}$ e $\forall V \in \mathcal{V}, \exists U_{V} \in \mathcal{U}, V \subset U_{V}$.

Definição 1.2.24. Dizemos que $X$ é paracompacto se toda cobertura aberta admite um refinamento aberto localmente finito. Se toda cobertura aberta admitir refinamento aberto dois-a-dois disjunto, dizemos que $X$ é ultraparacompacto

É um resultado básico em topologia o fato de que todo espaço compacto Hausdorff é normal. De maneira parecida, podemos enfraquecer a hipótese de compacidade para paracompacidade e obter o mesmo resultado. Por este motivo, os resultados sobre normalidade de produtos caixa estão muitas vezes relacionados à paracompacidade.

Neste texto, trabalharemos na maioria das vezes com espaços regulares. Supondo que determinado espaço topológico é regular, obtemos várias equivalências para a paracompacidade de tal espaço. Algumas destas equivalências estão demonstradas, por exemplo, em Engelking [Eng89] e em Kelley [Kel75]. Porém apenas utilizaremos a seguinte equivalência: 
Teorema 1.2.25 (Michael [Mic53]). Seja X um espaço topológico regular. Então são equivalentes as afirmações:

\section{$i X$ é paracompacto}

ii Toda cobertura aberta de $X$ admite refinamento aberto $\sigma$-localmente finito

Demonstração. É fácil ver que $\mathrm{i} \Rightarrow \mathrm{ii}$, pois toda família localmente finita é, em particular, $\sigma$-localmente finita. Para provar $i i \Rightarrow i$, seja $\mathcal{C}$ uma cobertura aberta de $X$. Seja $\mathcal{U}=\bigcup_{n \in \omega} \mathcal{U}_{n}$ um refinamento aberto de $\mathcal{C}$ tal que para cada $n \in \omega, \mathcal{U}_{n}$ é uma família localmente finita em $X$. Para cada $n \in \omega$, e cada $x \in \bigcup \mathcal{U}_{n}$ usamos a regularidade de $X$ para escolher dois abertos $U_{x}$ e $V_{x}$ tais que $x \in V_{x} \subset \overline{V_{x}} \subset U_{x} \in \mathcal{U}_{n}$. Definimos $\mathcal{V}_{n}=\left\{V_{x}: x \in \bigcup \mathcal{U}_{n}\right\}$. Fixando $m \in \omega$ e $y \in \bigcup \mathcal{U}_{m}$, definimos

$$
W_{m, y}=V_{y} \backslash \bigcup_{n<m}\left(\bigcup_{V \in \mathcal{V}_{n}} \bar{V}\right)
$$

Note que $\mathcal{V}_{n}$ é localmente finita, portanto $\bigcup_{V \in \mathcal{V}_{n}} \bar{V}=\overline{\bigcup \mathcal{V}_{n}}$ é fechado, e assim $W_{m, y}$ é aberto. Temos assim que $\mathcal{W}=\left\{W_{y}: m \in \omega, y \in \bigcup \mathcal{U}_{m}\right\}$ é um refinamento aberto localmente finito de $\mathcal{C}$, como queríamos.

Introduzimos agora uma ferramenta que usaremos diversas vezes para constatar que um espaço é paracompacto. Basicamente, tal resultado é uma generalização do resultado de que todo espaço regular Lindelöf é paracompacto. Para facilitar a notação, vamos introduzir a seguinte definição:

Definição 1.2.26. Seja $\kappa$ um cardinal infinito. Dizemos que um espaço topológico $X$ é

- $\kappa$-Lindelöf se toda cobertura aberta de $X$ admite uma subcobertura de cardinalidade menor ou igual a $\kappa$.

- um $P_{\kappa}$-espaço se qualquer intersecção de uma quantidade menor do que $\kappa$ de abertos é aberta. Alguns autores também utilizam a nomenclatura $\kappa$-aberto. 
Observação 1.2.27. Dizer que $X$ é $\kappa$-Lindelöf significa $L(X) \leq \kappa$. Note também que por definição, todo espaço topológico é um $P_{\aleph_{0}}$-espaço, e que todo espaço $\kappa$-Lindelöf, com $\kappa>\aleph_{0}$ é um P-espaço.

Lema 1.2.28. Seja $X$ um espaço topológico regular. Se existe um cardinal infinito $\kappa$ tal que $X$ é $P_{\kappa}$ espaço $\kappa$-Lindelöf, então $X$ é paracompacto.

Demonstração. Se $\kappa=\aleph_{0}$, temos o resultado tradicional de que todo espaço de Lindelöf regular é paracompacto. Mais especificamente, ele é corolário do teorema 1.2.25: se $X$ é regular Lindelöf, dada uma cobertura aberta, sua subcobertura enumerável é um refinamento $\sigma$-localmente finito, portanto $X$ é paracompacto.

Se $\kappa>\aleph_{0}$ e $X$ é um espaço nas condições do enunciado do teorema, então $X$ é em particular um P-espaço. Pela proposição 1.2.8, temos que $X$ é zero dimensional. Seja $\mathfrak{R}$ uma cobertura aberta de $X$. Podemos então construir um refinamento $\mathfrak{U}$ de $\mathfrak{R}$ formado por conjuntos abertos fechados. Como $X$ é um espaço $\kappa$-Lindelöf, temos uma subcobertura $\mathfrak{U}^{\prime}$ de $\mathfrak{U}$, de cardinalidade $\kappa$. Considerando $\kappa$ como um ordinal, podemos indexar $\mathfrak{U}^{\prime}$ por $\kappa$, isto é, $\mathfrak{U}^{\prime}=$ $\left\{U_{\alpha} \in \mathfrak{U}: \alpha<\kappa\right\}$. Temos $\mathfrak{R}^{\prime}=\left\{U_{\alpha} \backslash \bigcup_{\beta<\alpha} \overline{U_{\beta}}: \alpha<\kappa\right\}$ um refinamento dois a dois disjunto de $\mathfrak{R}$, o qual é aberto pois $X$ é $P_{\kappa}$ espaço. Portanto, $X$ é de fato paracompacto.

O lema a seguir servirá de ferramenta para a demonstração do teorema 3.1.5. Tal lema mostra que a paracompacidade é preservada por funções contínuas fechadas.

Lema 1.2.29. Seja $F: X \longrightarrow Y$ uma função contínua fechada e sobrejetora entre dois espaços topológicos regulares. Temos então:

1. Se $X$ é paracompacto, então $Y$ é também.

2. Se $Y$ é paracompacto e a pré-imagem por $F$ de cada ponto de $Y$ for Lindelöf , então $X$ é paracompacto.

Demonstração. A demonstração do item 1 pode ser vista em [Mic56]. Para o segundo item, seja $\mathcal{U}$ uma cobertura aberta de $X$. Para cada $y \in Y$, 
usando a hipótese de que $F^{-1}(\{y\})$ é Lindelöf, podemos obter uma família $\left\{U_{n}^{y}: n \in \omega\right\} \subset \mathcal{U}$ tal que $F^{-1}(\{y\}) \subset \bigcup_{n \in \omega} U_{n}^{y}$. Para cada $y \in Y$, definimos:

$$
V^{y}=\left\{z \in Y: F^{-1}(\{z\}) \subset \bigcup_{n \in \omega} U_{n}^{y}\right\}
$$

Pela escolha dos $U_{n}^{y}$, temos que $y \in V^{y}$. Notemos que o complementar de $V^{y}$ pode ser escrito como:

$$
\begin{aligned}
Y \backslash V^{y} & =\left\{z \in Y: F^{-1}(\{z\}) \not \subset \bigcup_{n \in \omega} U_{n}^{y}\right\} \\
& =\left\{z \in Y: \exists p \in F^{-1}(\{z\}), p \notin \bigcup_{n \in \omega} U_{n}^{y}\right\} \\
& =F\left(X \backslash V^{y}\right)
\end{aligned}
$$

Assim, como $F$ é fechada, podemos ver que $V^{y}$ é aberto. Usamos a paracompacidade de $Y$ para obter um refinamento $\mathcal{W}=\left\{W^{y}: y \in Y\right\}$ da cobertura aberta $\left\{V^{y}: y \in Y\right\}$ de tal forma que $\forall y \in Y, W^{y} \subset V^{y}$. Com isso, definimos para cada $n \in \omega$ a família

$$
R_{n}=\left\{U_{n}^{y} \cap F^{-1}\left(W^{y}\right): y \in Y\right\}
$$

Note que $R_{n}$ é uma família localmente finita: para cada $x \in \bigcup R_{n}$, seja $O_{x}$ a vizinhança aberta de $F(x)$ que testemunha que $\mathcal{W}$ é localmente finita (isto é, $O_{x}$ intercepta apenas um número finito de elementos de $\mathcal{W}$ ). Temos então que $U_{n}^{F(x)} \cap F^{-1}\left(O_{x}\right)$ é uma testemunha de que $R_{n}$ é localmente finito. Concluímos que $\mathcal{U}$ tem um refinamento $\sigma$-localmente finito e portanto $X$ é paracompacto (teorema 1.2.25).

\subsubsection{Espaços $\kappa$-metrizáveis}

Outra maneira de verificar que um espaço é paracompacto é o conceito de espaço $\kappa$-metrizável. Scott Williams define tal conceito no contexto uniformidades em [Wil84]. No entanto, utilizaremos a definição encontrada, por exemplo, em [Roi11].

Definição 1.2.30. Seja $\kappa$ um ordinal e $X$ um espaço topológico. Dizemos 
que $X$ é $\kappa$-metrizável se cada ponto $x \in X$ possui uma base local $\mathcal{B}_{x}=$ $\left\{u_{x, \alpha}: \alpha<\kappa\right\}$ tal que, dados dois pontos distintos $x, y \in X$ e dois ordinais $\alpha \leq \beta<\kappa$, temos:

1. $y \in u_{x, \alpha} \Rightarrow u_{y, \beta} \subset u_{x, \alpha}$

2. $y \notin u_{x, \alpha} \Rightarrow u_{y, \beta} \cap u_{x, \alpha}=\emptyset$

Note que, como este conceito é definido localmente, temos que $\kappa$-metrizabilidade é hereditária.

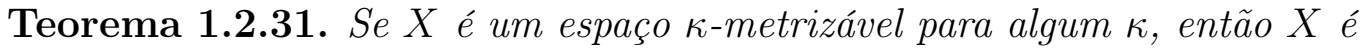
hereditariamente ultraparacompacto.

Demonstração. Seja $\mathcal{U}$ uma cobertura aberta de um espaço $\kappa$-metrizável $X$. Fixado $x \in X$, seja $\mathcal{U}_{x}$ uma base local como na definição 1.2.30. Seja $\alpha_{x}=\min \left\{\beta \in \kappa: \exists u \in \mathcal{U}, u_{x, \beta} \subset u\right\}$. Definimos então $u_{x}=u_{x, \alpha_{x}}$, e vamos mostrar que $\mathcal{U}^{\prime}=\left\{u_{x}: x \in X\right\}$ é uma partição de $X$.

Sejam $x, y \in X$ pontos distintos. Podemos supor $\alpha_{x} \leq \alpha_{y}$. Vamos dividir em dois casos:

Se $y \notin u_{x}$, pelo item 2 da definição 1.2.30, então $u_{x} \cap u_{y}=\emptyset$, como gostaríamos.

Se $y \in u_{x}$, pela contra-positiva do item 2, temos que $x \in u_{y, \alpha_{x}}$. Logo, pelo item 1, $u_{x} \subset u_{y, \alpha_{x}}$. Por outro lado, $y \in u_{x}$ e o item 1 também implicam que $u_{y, \alpha_{x}} \subset u_{x}$. Portanto, temos $u_{y, \alpha_{x}}=u_{x}$. De tal forma, pela definição de $\alpha_{y}$, concluímos que $\alpha_{y}=\alpha_{x}$, e portanto, $u_{x}=u_{y}$, também como gostaríamos.

Por fim, observe que a hereditariedade decorre de que a $\kappa$-metrizabilidade é uma propriedade hereditária. 


\section{Capítulo 2}

\section{Comparações com o produto de Tychonoff}

Neste capítulo, apresentamos resultados sobre o produto caixa com o propósito de compará-lo com o produto de Tychonoff. Começamos apresentando as definições de ambos os produtos, e verificando algumas propriedades básicas.

Na seção seguinte, veremos como o produto caixa, diferentemente do produto de Tychonoff, pode ser utilizado na construção de espaços discretamente gerados.

Por fim, estudaremos o conjunto $C(X)$ das funções contínuas de $X$ em $\mathbb{R}$ relativamente a dois espaços topológicos que este pode gerar: $C_{p}(X) e$ $C_{\square}(X)$, associados ao produto de Tychonoff e caixa, respectivamente. $\mathrm{Na}$ mesma seção, veremos as relações entre o espaço $C_{\square}(X)$ e espaços quase- $\omega$ resolúveis, além de sua relação com cardinais mensuráveis.

\subsection{Definições}

Começamos apresentando as definições dos produtos que utilizaremos neste texto.

Definição 2.1.1. Sejam $X_{i}$ espaços topológicos para cada $i \in I$, e seja $\prod_{i \in I} X_{i}$. Se $A \subset \prod_{i \in I} X_{i}$ é da forma $A=\prod_{i \in I} A_{i}$, como $A_{i} \subset X_{i}$ para cada 
$i \in I$, chamamos de suporte de A o conjunto $\operatorname{spt}(A)=\left\{i \in I: A_{i} \neq X_{i}\right\}$

Definição 2.1.2. Para cada $i \in I$, seja $X_{i}$ um espaço topológico. Definimos $o$ produto de Tychonoff da família $\left\{X_{i}: i \in I\right\}$ como o espaço topológico formado pelo produto cartesiano $\prod_{i \in I} X_{i}$ equipado com a topologia gerada pela seguinte família:

$$
\left\{\prod_{i \in I} V_{i}: V_{i} \text { é aberto em } X_{i} \text { e spt }\left(\prod_{i \in I} V_{i}\right) \text { é finito }\right\}
$$

Nos referimos aos elementos desta família por abertos básicos. Denotaremos tal espaço topológico por $\mathrm{T}_{i \in I} X_{i}$, e a topologia em questão por $\tau_{T}$. No caso em que existe um espaço $X$ tal que $\forall i \in I, X_{i}=X$, denotamos $\mathrm{T}^{I} X$.

Definição 2.1.3. Para cada $i \in I$, seja $X_{i}$ um espaço topológico. Definimos $o$ produto de caixa da família $\left\{X_{i}: i \in I\right\}$ como o espaço topológico formado pelo produto cartesiano $\prod_{i \in I} X_{i}$ equipado com a topologia gerada pela seguinte família:

$$
\left\{\prod_{i \in I} V_{i}: V_{i} \text { é aberto em } X_{i}\right\}
$$

Nos referimos a um elemento de tal família por aberto básico como no produto de Tychonoff, ou também por caixa aberta. Da mesma forma, denominamos caixa fechada um produto qualquer de conjuntos fechados.

Denotaremos tal espaço topológico por $\square_{i \in I} X_{i}$, e a topologia em questão por $\tau_{\square}$. No caso em que existe um espaço $X$ tal que $\forall i \in I, X_{i}=X$, denotamos $\square^{I} X$.

Note que todo aberto no produto de Tychonoff é em particular aberto no produto caixa. Em outras palavras, dizemos que $\tau_{\square}$ é uma topologia mais fina do que a topologia $\tau_{T}$.

Existe também um produto intermediário entre o de Tychonoff e o da caixa, o $\kappa$-produto caixa.

Definição 2.1.4. Sejam I um conjunto infinito e $X_{i}$ um espaço topológico para cada $i \in I$. Seja $\kappa$ um cardinal infinito. Definimos o $\kappa$-produto caixa 
da família $\left\{X_{i}: i \in I\right\}$ como o espaço topológico formado pelo produto cartesiano $\prod_{i \in I} X_{i}$ equipado com a topologia gerada pela seguinte família:

$$
\left\{\prod_{i \in I} V_{i}: V_{i} \text { é aberto em } X_{i} \text { e }\left|\operatorname{spt}\left(\prod_{i \in I} V_{i}\right)\right|<\kappa\right\}
$$

Novamente podemos nos referir a um elemento desta familia por aberto básico ou então por $\kappa$-caixa aberta.

Denotaremos tal espaço topológico por $\kappa \square_{i \in I} X_{i}$, e a topologia em questão por $\tau_{\kappa \square .}$. No caso em que existe um espaço $X$ tal que $\forall i \in I, X_{i}=X$, denotamos $\kappa \square^{I} X$.

Note que tanto o produto de Tychonoff quanto o produto caixa são casos particulares do $\kappa$-produto caixa quando $\kappa=\aleph_{0}$ e $\kappa>|I|$, respectivamente.

\subsection{Propriedades básicas}

O produto de Tychonoff e o produto caixa compartilham várias propriedades básicas. Expomos aqui algumas delas.

Projeções Uma delas é o fato de que em ambos os produtos as projeções são contínuas. Mais do que isso, o produto de Tychonoff é a topologia menos fina com tal propriedade. Por isso dizemos que a topologia de Tychonoff é a topologia em $\prod_{i \in I} X_{i}$ induzida pela família de funções $\left\{\pi_{i}: i \in I\right\}$, e desse fato decorrem algumas das boas propriedades que justificam a escolha do produto de Tychonoff como produto usual.

Proposição 2.2.1. Sejam $X_{i}$ espaços topológicos para cada $i \in I$, e considere o conjunto $\prod_{i \in I} X_{i}$. A topologia em $\prod_{i \in I} X_{i}$ induzida pelas projeções $\pi_{i}$ : $\prod_{i \in I} X_{i} \longrightarrow X_{i}$ é precisamente a topologia do produto de Tychonoff. Logo, uma outra topologia em $\prod_{i \in I} X_{i}$ torna as projeções contínuas se e somente se for mais fina do que $\tau_{T}$.

Demonstração. Seja $\tau$ a topologia em $\prod_{i \in I} X_{i}$ gerada pelas projeções, e seja $\tau_{i}$ a topologia de $X_{i}$ para cada $i \in I$. Fixemos $j \in I$. Seja $V$ um aberto em 
$X_{j}$. Note que $\pi_{j}^{-1}(V)=\prod_{i \in I} V_{i}$, onde $V_{j}=V$ e $V_{i}=X_{i}$ para $i \neq j$. Portanto, para que $\pi_{j}$ seja contínua, precisamos que $\pi_{j}^{-1}(V) \in \tau$. Como queremos que todas as projeções sejam contínuas ao mesmo tempo, precisamos que essa inclusão valha para todo $i \in I$ e para todo aberto de $\tau_{i}$. Logo $\tau$ é gerado pela pré-base $\mathcal{B}=\left\{\pi_{i}^{-1}(V): i \in I, V \in \tau_{i}\right\}$, isto é, o conjunto $\mathcal{B}^{\prime}$ constituído de todas as intersecções finitas de elementos de $\mathcal{B}$ é uma base para $\tau$. Só que $\mathfrak{B}^{\prime}$ nada mais é do que a base do produto de Tychonoff.

Uma consequência da proposição 2.2.1 é que, dada uma família $\left\{A_{i} \subset\right.$ $\left.X_{i}: i \in I\right\}$, o produto de Tychonoff $\prod_{i \in I} A_{i}$ coincide com a topologia de subespaço de $\prod_{i \in I} A_{i}$ herdada de $\prod_{i \in I} X_{i}$, pois a topologia de subespaço nada mais é do que a topologia induzida pela função inclusão. Tal resultado também vale para o produto caixa.

Axiomas de separação Outra característica em comum do produto caixa e de Tychonoff é que ambos preservam a maioria dos axiomas de separação. Em ambos os produtos são preservados os axiomas $T_{0}, T_{1}, T_{2}, T_{3}, T_{3 \frac{1}{2}}$, e a demonstração para cada caso é semelhante. Como ambos os produtos são casos particulares do $\kappa$-produto caixa, demonstraremos para tal caso geral.

Proposição 2.2.2. Seja $X_{i}$ um espaço topológico $T_{n}$ para cada $i \in I$, com $n=0,1,2,3,3 \frac{1}{2}$. Então $\kappa \square_{i \in I} X_{i}$ é um espaço topológico $T_{n}$ para qualquer $\kappa$ infinito.

Demonstração. Para $n=0,1,2,3$, a demonstração é semelhante. Exibimos então o caso $n=3$, e para isto utilizaremos a equivalência de $T_{3}$ apresentada na proposição 1.2.6. Seja $x \in \prod_{i \in I} X_{i}$, e seja também um aberto básico $V \subset \prod_{i \in I} X_{i}$. Para cada $i \in \operatorname{spt} V$, usamos o fato de $X_{i}$ ser espaço topológico $T_{3}$ para encontrar um aberto $U_{i} \subset X_{i}$ tal que $x_{i} \in U_{i} \subset \overline{U_{i}} \subset V_{i}$. Para $i \notin \operatorname{spt} V$, definimos $U_{i}=X_{i}$. Assim, temos $U=\prod_{i \in I} U_{i}$ aberto em $\kappa \square_{i \in I} X_{i}$.

Já o caso $n=3 \frac{1}{2}$ é mais complicado e técnico. Como neste texto trabalharemos na maioria dos casos com produtos enumeráveis, exibimos a demonstração para o caso em que $I=\omega$. Seja $F \subset \kappa \square_{i \in I} X_{i}$ um conjunto fechado, e seja $x_{0} \in \kappa \square_{i \in I} X_{i} \backslash F$. Podemos escolher uma vizinhança básica $V=\prod_{i \in I} V_{i}$ de $x_{0}$ tal que $V \cap F=\emptyset$. Para cada $i \in I$, como $X_{i}$ é $T_{3 \frac{1}{2}}$, 
construímos uma função contínua $f_{i}: X_{i} \longrightarrow \mathbb{I}$ tal que $f_{i}\left(x_{i}\right)=0$ e $f(y)=1$ para todo $y \in X_{i} \backslash V_{i}$ (caso este seja não vazio). Definimos então a função $f: \kappa \square_{i \in I} X_{i} \longrightarrow \mathbb{I}$ definida por $f(x)=\frac{1}{2} \sum_{i=0}^{\infty} \frac{f_{i}\left(x_{i}\right)}{2^{i}}$ para cada $x \in \kappa \square_{i \in I} X_{i}$. Para cada $x \in \kappa \square_{i \in I} X_{i}$, a série $\sum_{i=0}^{\infty} \frac{f_{i}\left(x_{i}\right)}{2^{i}}$ converge. Além disso, temos que $f\left(x_{0}\right)=0$, além do que para todo $p \in F$ temos $p_{i} \notin V_{i}$ para cada $i \in I$, portanto $f(p)=\frac{1}{2} \sum_{i=0}^{\infty} \frac{1}{2^{i}}=1$. Logo $\kappa \square_{i \in I} X_{i}$ satisfaz $T_{3 \frac{1}{2}}$.

Observação 2.2.3. Existe um truque [vD80] para o caso geral da preservação de espaços completamente regulares. Pode-se verificar que um espaço é completamente regular se e somente se o mesmo é homeomorfo a um subesçao de um grupo topológico. A partir do produto direto de grupos podemos mostrar que o $\kappa$ produto de grupos topológicos também é um grupo topológico. Outra opção para o mesmo truque seria utilizar uniformidades ao invés de grupos topológicos. Trata-se de uma consequência direta do teorema A.2.5 e da proposição A.3.4.

Notemos que, no entanto, a preservação não chega à normalidade, nem mesmo no caso finito, onde o produto caixa e o de Tychonoff coincidem. O exemplo mais famoso deste fato é a reta de Sorgenfrey, a qual é um espaço normal cujo quadrado não preserva a normalidade. Veremos outros exemplos de tal fato no capítulo 4. O problema da normalidade está relacionado com o da paracompacidade, o qual será discutido no capítulo 3.

Fecho A última propriedade em comum entre os dois produtos que apresentaremos é o fato de que ambos preservam o fecho dos fatores. Além de caracterizar o fecho nos produtos, tal propriedade garante que o produto qualquer de espaços fechados é fechado, o que justifica a nomenclatura caixa fechada (definição 2.1.3).

Proposição 2.2.4. Sejam $\kappa$ um cardinal infinito e $A_{i} \subset X_{i}$ para cada $i \in I$. Temos então:

$$
\overline{\kappa \square_{i \in I} A_{i}}=\kappa \square_{i \in I} \overline{A_{i}}
$$

Demonstração. Para mostrar que $\overline{\kappa \square_{i \in I} A_{i}} \subset \kappa \square_{i \in I} \overline{A_{i}}$, vamos mostrar que $\kappa \square_{i \in I} \overline{A_{i}}$ é fechado. Seja $p \in \kappa \square_{i \in I} X_{i}$ tal que $p \notin \kappa \square_{i \in I} \overline{A_{i}}$. Temos que existe 
algum $j \in I$ tal que $p_{j} \notin \overline{A_{j}}$, para o qual podemos encontrar um aberto $V_{j}$ tal que $p_{j} \in V_{j} \subset X_{j} \backslash A_{j}$. Temos então que $V=\prod_{i \in I} V_{i}$, onde $V_{i}=X_{i}$ para todo $i \neq j$ é uma vizinhança aberta de $p$ que não intersecciona $\kappa \square_{i \in I} \overline{A_{i}}$, como gostaríamos.

Vamos agora mostrar que $\overline{\kappa \square_{i \in I} A_{i}} \supset \kappa \square_{i \in I} \overline{A_{i}}$. Consideremos $x \in \kappa \square_{i \in I} \overline{A_{i}}$. Seja $V=\prod_{i \in I} V_{i}$ uma vizinhança aberta básica de $x$. Como $x_{i} \in \overline{A_{i}}$, podemos encontrar $y_{i} \in V_{i} \cap A_{i}$. Assim, temos $y=\left(y_{i}\right)_{i \in I} \in V \cap \prod_{i \in I} A_{i}$, logo $x \in \overline{\kappa \square_{i \in I} A_{i}}$, como queríamos.

Note que, além de demonstrar que o produtos de fechado é fechado, tal demonstração também mostra que a recíproca de tal afirmação é verdadeira, isto é, que se um produto de subespaços é fechado, então todos os fatores são fechados. Outro corolário da proposição é o seguinte:

Corolário 2.2.5. Seja $D_{i}$ um subespaço não vazio de $X_{i}$ para cada $i \in I$. Então $\prod_{i \in I} D_{i}$ é denso em $\kappa \square_{i \in I} X_{i}$ se e somente se para cada $i \in I, D_{i}$ for denso em $D_{i}$.

Note também que a proposição 2.2.4 junto com a definição de produto caixa garante que o produto caixa preserva a zero dimensionalidade.

Veremos agora propriedades que distinguem os produtos.

Convergência de sequências Um argumento tradicional para se justificar a escolha do produto de Tychonoff ao invés do produto caixa como produto usual de topologias é a convergência de sequências. Por exemplo, considere o produto cartesiano de $\omega$ cópias da reta real $\mathbb{R}$. É um resultado básico e intuitivo de análise real que a sequência $\left(\frac{1}{n}\right)_{n \in \omega}$ converge para 0 em $\mathbb{R}$. Pode se pensar que esse resultado seja preservado com o seu análogo em produto caixa, o que não acontece, como mostra o próximo exemplo:

Definição 2.2.6. Dados dois conjuntos $X$ e I definimos a função $\widehat{-}: X \rightarrow$ $X^{I}$ por $\widehat{x}=(x)_{i \in I}$ para todo $x \in X$.

Exemplo 2.2.7. A sequência $\left(\widehat{\left(\frac{1}{n}\right)}\right)_{n \in \omega} e m \square^{\omega} \mathbb{R}$ não converge para $\widehat{0}$. 
Este exemplo é um caso específico da próxima proposição, a qual mostra que o produto caixa é muito mais pobre em termos de sequências convergentes.

Proposição 2.2.8. Seja $X$ um espaço topológico $T_{1}$. Temos então que as únicas sequências convergentes em $\square^{\omega} X$ são as eventualmente constantes (isto é, sequências para as quais existe $n_{0} \in \omega$ tal que ela é constante a partir de $\left.n_{0}\right)$.

Demonstração. Sejam $\left(a_{n}\right)_{n \in \omega}$ uma sequência em $\square^{\omega} X$ e $a \in \square^{\omega} X$ com a condição de que $\left(a_{n}\right)_{n \in \omega}$ não é eventualmente constante igual a $a$, isto é, para cada $n \in \omega$ existe $n_{0}>n$ tal que $a_{n_{0}} \neq a$.

Como $X$ é $T_{1}$, para cada $i \in \omega$ tal que $a_{i} \neq a$ escolhemos $V_{i} \subset X$ aberto tal que $a \in V_{i}$ e $a_{i} \notin V_{i}$. Já para $i \in \omega$ tal que $a_{i}=a$, fixamos $V_{i}=X$. Assim, temos que $V=\prod_{i \in \omega} V_{i}$ é uma vizinhança de $a$ tal que $\left\{n \in \omega: a_{n} \notin V\right\}$ é infinito. Portanto, temos que $\left(a_{n}\right)_{n \in \omega}$ não converge para $a$.

Note que o suporte do aberto $V$ da demonstração anterior era infinito, portanto esse mesmo conjunto não seria aberto no produto de Tychonoff.

Bases As definições 2.1.2, 2.1.3 e 2.1.4 de produtos de espaços topológicos podem ser reescritas equivalentemente considerando apenas produtos de abertos básicos, fixadas bases quaisquer para cada um dos fatores envolvidos. De tal forma, para qualquer cardinal infinito $\kappa$, temos que $w\left(\kappa \square_{i \in I} X_{i}\right) \leq \prod_{i \in I} w\left(X_{i}\right)$. Podemos assim majorar o peso (e consequentemente a densidade e o grau de Lindelöff) de um produto através do peso dos fatores, o que normalmente não é uma boa majoração.

Mais do que isso, como o produto caixa compreende uma quantidade de abertos muito maior que o produto de Tychonoff, é natural que o peso também seja maior no produto caixa. Podemos observar tal aumento nos produtos de espaços que satisfaçam o primeiro e o segundo axioma da enumerabilidade (definições 1.2.17 e 1.2.20). Enquanto que o produto de Tychonoff enumerável preserva ambos os axiomas [Eng89], produtos caixa infinitos normalmente não os satisfazem. 
Proposição 2.2.9 (Rudin [Rud75]). Seja $X_{i}$ um espaço Hausdorff não discreto para cada $i \in \omega$. Então $\square_{i \in I} X_{i}$ não é primeiro enumerável.

Demonstração. Seja $x \in \square_{i \in \omega} X_{i}$ um ponto tal que para todo $i \in \omega, x_{i}$ não é ponto isolado de $X_{i}$. Seja $\mathcal{V}$ uma base local de $x$, e suponha por absurdo $\mathcal{V}=$ $\left\{V_{n}=\prod_{i \in \omega} V_{n, i}: n \in \omega\right\}$, onde para todo $(n, i) \in \omega^{2}$ temos $V_{n, i} \subset X_{i}$. Para cada $i \in \omega$, podemos supor a sequência $\left(V_{n, i}\right)_{n \in \omega}$ estritamente decrescente quanto à ordem $\subset$. Temos então que $V=\prod_{i \in \omega} V_{i, i}$ é uma vizinhança aberta de $x$ que não contém nenhum elemento de $\mathcal{V}$, contrariando o fato de que $\mathcal{V}$ é uma base local de $x$. Logo $|\mathcal{V}|>\aleph_{0}$, como queríamos.

Como anteriormente, o motivo de tal demonstração não funcionar com o produto de Tychonoff é que, em tal caso, o conjunto $V$ não seria aberto, tão pouco vizinhança de $x$.

O seguinte corolário decorre do fato de que o caráter de um espaço ser menor que o seu peso, visto que uma base de um espaço contém bases locais para todos os seus pontos.

Corolário 2.2.10. Seja $X_{i}$ um espaço Hausdorff não discreto para cada $i \in \omega$. Então $\square_{i \in I} X_{i}$ não é segundo enumerável.

Compacidade Uma das principais propriedades do produto de Tychonoff é o Teorema de Tychonoff, o qual é inclusive equivalente ao axioma da escolha. O Teorema diz que o produto Tychonoff qualquer de espaços compactos é compacto. Em contrapartida, tal resultado quase sempre é falso para produtos caixa. Mais do que isso, os produtos caixa em geral não são compactos, o que é um dos grandes problemas para se trabalhar com eles.

Teorema 2.2.11 (Williams [Wil84]). Seja $X_{i}$ um espaço Hausdorff e não discreto para cada $i \in I,|I| \geq \aleph_{0}$, então $\square_{i \in I} X_{i}$ não é localmente compacto.

Demonstração. Para cada $i \in I$, sejam $p_{i}$ um ponto não isolado de $X_{i}$ e $G=\prod_{i \in I} G_{i}$ uma vizinhança básica de $p=\left(p_{i}\right)_{i \in I}$. Como $p_{i}$ não é isolado, escolhemos $x_{i} \in G_{i}$ tal que $x_{i} \neq p_{i}$. Pela regularidade de $X_{i}$, podemos construir abertos disjuntos $U_{i}, V_{i}$ tais que $p_{i} \in U_{i}$ e $x_{i} \in V_{i}$. A partir disso, 
consideremos o conjunto $A=\prod_{i \in I}\left\{p_{i}, x_{i}\right\} \subset G$. Consideremos então a seguinte cobertura aberta de $A$ :

$$
\mathfrak{H}=\left\{\prod_{i \in I} K_{i}: \forall i \in I, K_{i} \in\left\{G_{i} \cap U_{i}, G_{i} \cap V_{i}\right\}\right\}
$$

Note que $|\mathfrak{H}|=2^{|I|}>\aleph_{0}$, além de que $\mathcal{H}$ é dois a dois disjunta. Temos assim que $\mathcal{H}$ é uma cobertura aberta de $A$ que não admite subcobertura finita. Então $A$ não é compacto. Como $A$ é fechado (proposição 2.2.4), $G$ também não é compacto. Concluímos que $\square_{i \in I} X_{i}$ não é localmente compacto.

Note que esta demonstração também garante que $\square_{i \in I} X_{i}$ não é separável. De fato, como a família $\mathfrak{H}$ é dois a dois disjunta, temos que $d\left(\square_{i \in I} X_{i}\right) \geq$ $c\left(\square_{i \in I} X_{i}\right) \geq|\mathfrak{H}|=2^{|I|}>\aleph_{0}$.

A falta da compacidade nos produtos caixa levou à investigação da $p a-$ racompacidade em tais produtos. O estudo desta propriedade se tornou o principal tema relativos ao produto caixa, o qual veremos no capítulo 3.

Conexidade Inicialmente, mostraremos que o produto de Tychonoff se comporta muito bem quanto à preservação da conexidade. Para isso, usaremos a demonstração por Engelking [Eng89]. Contudo, precisaremos antes de alguns resultados básicos sobre conexidade.

Lema 2.2.12. Seja $\mathcal{C} \subset \mathcal{P}(X)$ uma família de subespaços conexos de um espaço topológico $X$. Se $\bigcap \mathcal{C} \neq \emptyset$, então $\bigcup \mathcal{C}$ é conexo.

Demonstração. Sejam $X$ e $\mathcal{C}$ como no enunciado. Suponha $A, B$ abertos em $X$ tais que $\bigcup \mathcal{C} \subset A \cup B$ e $A \cap B \cap(\bigcup \mathcal{C})$. Fixemos $p \in \cap \mathcal{C}$. Suponhamos que $p \in A$. Para cada $C \in \mathcal{C}$, temos que $p \in C \cap A$. Como $C$ é conexo, temos que $C \subset A$. Logo, temos que $\bigcup \mathcal{C} \subset A$ e $(\bigcup \mathcal{C}) \cap B=\emptyset$, e portanto $\bigcup \mathcal{C}$ é conexo.

Lema 2.2.13. Seja $X$ um espaço topológico. Se qualquer par de pontos de $X$ pode ser ligado por um conjunto conexo, isto é, dados $x, y \in X$ existe um conjunto conexo $C \subset X$ tal que $x, y \in C$, então $X$ é conexo. 
Demonstração. Fixemos $x \in X$. Para cada $y \in X$, definimos $C_{y}$ o conjunto conexo que liga $x$ e $y$, como no enunciado. Temos então que $\{x\}=\bigcap_{y \in X} C_{y}$ e $X=\bigcup_{y \in X} C_{y}$, portanto pelo lema 2.2.12, $X$ é conexo.

Lema 2.2.14. Dado um espaço topológico $X$, se $X$ contém um conjunto denso conexo, então $X$ é conexo

Demonstração. Seja $D \subset X$ um denso conexo, e sejam $A, B \subset X$ abertos tais que $A \cup B=X$ e $A \cap B=\emptyset$. Note que $A \cup B \supset D$. Então, como $D$ é conexo, podemos supor, sem perda de generalidade, que $D \subset A$ e $B \cap D=\emptyset$. Como $D$ é denso, temos que a $B \cap D \neq \emptyset$ a menos que $B=\emptyset$. Logo, concluímos que de fato $B=\emptyset$ e $A=X$. Portanto, $X$ é conexo.

Teorema 2.2.15. O produto de Tychonoff de espaços conexos não vazios é conexo.

Demonstração. Primeiramente, vamos mostrar o caso finito. Sejam $X$ e $Y$ espaços conexos não vazios. Dados $\left(x_{1}, y_{1}\right)$ e $\left(x_{2}, y_{2}\right)$ pontos quaisquer em $X \times Y$, este pode ser unido pelo conjunto $\left(X \times\left\{y_{1}\right\}\right) \cup\left(\left\{x_{2}\right\} \times Y\right)$, o qual é conexo pelo lema 2.2.12. Portanto, $X \times Y$ é conexo pelos lema 2.2.13. Por indução, estendemos o resultado para qualquer produto finito.

Consideramos então o caso infinito. Seja $X_{i}$ um espaço conexo não vazio para cada $i \in I, I$ infinito. Definimos $\mathfrak{J}=[I]^{<\aleph_{0}}$ o conjunto de todas as partes finitas de $I$. Fixamos também um $x \in \prod_{i \in I} X_{i}$ qualquer. Para cada $J \in \mathfrak{J}$, definimos $C_{J}=\prod_{i \in I} C_{i}$, onde $C_{i}=X_{i}$ para $i \in J$, e $C_{i}=\left\{x_{i}\right\}$ para $i \in I \backslash J$. Do caso finito, temos que $C_{J}$ é conexo, para todo $J \in \mathfrak{J}$. Como $x \in \bigcap_{J \in \mathfrak{J}} C_{J} \neq \emptyset$, temos que $\bigcup_{J \in \mathfrak{J}} C_{J}$ é conexo pelo lema 2.2.12. Além disso, note que $\bigcup_{J \in \mathfrak{J}} C_{J}$ é denso em $\prod_{i \in I} X_{i}$, logo $\prod_{i \in I} X_{i}$ é conexo pelo lema 2.2.14, como queríamos.

Já no caso do produto caixa, dificilmente obtemos produtos conexos. Como tal produto possui muito mais conjuntos abertos do que o produto de Tychonoff, basta adicionar um axioma de separação aos fatores para que o produto seja desconexo. 
Teorema 2.2.16 (Rudin [Rud75]). Seja $X_{i}$ um espaço regular para cada $i \in$ $\omega$. Então dado $x \in \square_{i \in \omega} X_{i}$, o conjunto $q(x)=\left\{p \in \square_{i \in I} X_{i}:\left|\left\{i: x_{i} \neq p_{i}\right\}\right|<\aleph_{0}\right\}$ é a componente conexa de $\square_{i \in \omega} X_{i}$ à qual $x$ pertence.

Demonstração. Seja $p \in \square_{i \in \omega} X_{i} \backslash q(x)$. Para cada par $(n, i)$ de números naturais, escolhemos um aberto $U_{n, i} \subset X_{i}$ tal que $x_{i} \in U_{n, i}, p_{i} \notin U_{n, i}$ caso $p_{i} \neq x_{i}$, e $U_{n, i} \supset \overline{U_{(n+1), i}}$ (esta última condição é possível graças à regularidade do espaço). Definimos então

$$
Y=\left\{y \in \square_{i \in \omega} X_{i}: \exists n,\left|\left\{i: y_{i} \notin U_{n, i}\right\}\right| \geq \aleph_{0}\right\}
$$

Por construção, temos claramente que $p \in Y$, porém $x \notin Y$. Notemos que $Y$ é aberto. Fixado $y \in Y$, escolhemos algum $n \in \omega$ tal que $S=$ $\left\{i \in \omega: y_{i} \notin U_{n, i}\right\}$ seja infinito. Considere para cada $i \in \omega$ a vizinhança aberta $V_{i}$ de $y_{i}$ dada por $V_{i}=X_{i} \backslash \overline{U_{(n+1), i}}$ para $i \in S$ ou por $V_{i}=X_{i}$ para $i \in \omega \backslash S$. Como $S$ é infinito, temos que $y \in V=\prod_{i \in \omega} V_{i} \subset Y$, comprovando que $Y$ é de fato aberto.

Além disso, temos que $Y$ é também fechado. Considere $z \in \square_{i \in \omega} X_{i} \backslash Y$. Pela definição de $Y$, podemos encontrar para cada $n \in \omega$ o menor natural $k_{n} \in \omega$ tal que para todo $i \geq k_{n}$ tenhamos $z \in U_{n, i}$. Como para cada $i \in \omega$ fixado escolhemos a sequência $\left(U_{n, i}\right)_{n \in \omega}$ de maneira decrescente, temos que a sequência $\left(k_{n}\right)_{n \in \omega}$ é crescente.

A partir de tal sequência, podemos construir outra da seguinte maneira: para cada $i \leq k_{0}$, definimos $a_{i}=0$. Em seguida, para $i \geq k_{0}$, se $a_{i}+1<$ $k_{a_{i}+1}$, definimos $a_{i+1}=a_{i}$. Caso contrário, definimos $a_{i+1}=a_{i}+1$. O fato de $\left(k_{n}\right)_{n \in \omega}$ ser crescente garante que $\left(a_{i}\right)_{i \in \omega}$ também o é. Definimos então $W_{i}=X_{i}$ para $i<k_{0}$, e $W_{i}=U_{a_{i}, i}$ para $i \geq k_{0}$. Temos assim que $z \in W=\prod_{i \in \omega} W_{i} \subset \square_{i \in \omega} X_{i} \backslash Y$. De fato, a sequência $\left(k_{n}\right)_{n \in \omega}$ garante que $z \in \prod_{i \in \omega} W_{i}$. Além disso, o fato de a sequência $\left(a_{i}\right)_{i \in \omega}$ ser crescente garante que $Y \cap W=\emptyset$, pois para cada $n \in \omega$, podemos encontrar $i_{n} \in \omega$ tal que $a_{i_{n}}>n$, de maneira que $W_{i}=U_{a_{i}, i} \subset U_{n, i}$ para todo $i>i_{n}$. Portanto, $Y$ é fechado.

Como $Y$ é uma vizinhança aberto-fechada de $p$ a qual não contém $x$, então $p$ pertence a uma componente conexa distinta da de $x$, então concluímos que 
a componente conexa à qual $x$ pertence está contida em $q(x)$.

Para finalizar, basta mostrar que o conjunto $q(x)$ é conexo. Notemos que tal conjunto nada mais é do que o conjunto $\bigcup_{J \in \mathfrak{J}} C_{J}$ como construído na demonstração do teorema 2.2.15, com $x$ fixado. Como demonstrado em tal teorema, temos que $q(x)=\bigcup_{J \in \mathfrak{J}} C_{J}$ é conexo.

Note que usaremos esse conjunto $q(x)$ no próximo capítulo para construir o produto nabla (definição 3.1.1). Inclusive, usaremos o mesmo argumento de diagonal que usamos para provar que o conjunto $Y$ é fechado para mostrar que o produto nabla é um P-espaço (lema 3.1.4). Além disso, decorre também da demonstração que $\square_{i \in \omega} X_{i}$ não é localmente conexo, pois qualquer vizinhança básica de um ponto $x$ contém um ponto $y$ que não pertence a $q(x)$.

Com este resultado, para que o produto caixa de espaços regulares seja não conexo, basta que não seja um produto trivial, no sentido de que uma quantidade infinita de fatores tenha mais do que 1 ponto. Note que a demonstração do teorema 2.2.15 não funciona para o produto caixa apenas pelo fato de que o conjunto $\bigcup_{J \in \mathfrak{J}} C_{J}$ não é denso em $\square_{i \in I} X_{i}$.

\subsection{Espaços discretamente gerados}

Como vimos até agora, muitas das propriedades preservadas pelo produto de Tychonoff não são preservadas pelo produto caixa. Veremos agora um exemplo de espaço que se comporta de maneira inversa: os espaços discretamente gerados, introduzidos por Dow, Tkachenko, Tkachuk e Wilson em [DTTW02]. Enquanto que o produto de Tychonoff de tais espaços não é necessariamente discretamente gerado, podemos construir tais espaços utilizando o produto caixa.

Definição 2.3.1. Dizemos que um espaço topológico $X$ é discretamente gerado se dados $A \subset X$ e $x \in \bar{A}$, então existe um conjunto discreto $D \subset A$ tal que $x \in \bar{D}$.

Proposição 2.3.2. Todo subespaço de um espaço discretamente gerado também é discretamente gerado. 
Demonstração. Seja $X$ um espaço discretamente gerado, e seja $S \subset X$. Seja $A \subset S$, e seja $p \in \bar{A}^{S}$. Note que $p \in \bar{A}^{X}$, portanto existe um discreto $D \subset A$ em $X$ tal que $p \in \bar{D}^{X}$. Seja $D^{\prime}=D \cap S$. Note que $D^{\prime} \subset A$ também é discreto e ${\overline{D^{\prime}}}^{S}=\bar{D}^{X} \cap S$, logo $p \in{\overline{D^{\prime}}}^{S}$, do que se conclui que $S$ é discretamente gerado.

Para a demonstração do exemplo 2.3.5, precisaremos do conceito de espaços maximais e submaximais. Voltaremos a utilizar tal conceito na seção 2.4

Definição 2.3.3. Seja $(X, \tau)$ um espaço denso-em-si-mesmo (isto é, $(X, \tau)$ não possui pontos isolados). $X$ é dito:

- maximal quando $\tau$ é a maior topologia densa-em-si-mesma sobre $X$, ou seja, dada qualquer topologia $\tau^{\prime}$ sobre $X$ com $\tau \subsetneq \tau^{\prime},\left(X, \tau^{\prime}\right)$ possui algum ponto isolado.

- submaximal se todo subconjunto denso de $X$ for aberto.

Apesar do nome, existem espaços topológicos maximais que não são submaximais. Considere um espaço topológico formado por 3 pontos distintos, e cujo único aberto não trivial consiste do conjunto formado por 2 destes pontos. Aliás, é possível encontrar exemplos como esse de espaços maximais não submaximais de qualquer cardinalidade. Contudo, basta a hipótese do espaço ser $T_{0}$ para que a maximalidade implique em submaximalidade (proposição 2.3.4). Tal hipótese faz sentido, uma vez que todo espaço submaximal é $T_{1}$, pois o complementar de qualquer ponto não isolado é denso.

Proposição 2.3.4. Todo espaço maximal $T_{0}$ é submaximal.

Demonstração. Seja $(X, \tau)$ um espaço maximal $T_{0}$. Vejamos primeiro que $(X, \tau)$ é $T_{1}$. Dado um ponto $p \in X$, suponhamos que $X \backslash\{p\} \notin \tau$. Como $(X, \tau)$ é maximal, se adicionarmos $X \backslash\{p\}$ à topologia $\tau$ (ou seja, se considerarmos a topologia $\tau^{\prime}$ gerada pela pré-base $\tau \cup\{X \backslash\{p\}\}$ ), teremos um ponto isolado. Isto significa que existe algum ponto $q \in X$ e um aberto $U \in \tau$ tais que $(X \backslash\{p\}) \cap U=\{q\}$. Temos então que $U \subset\{p, q\}$, pois se existisse algum $z \in U$ tal que $z \neq p, q$, então teríamos $z \in(X \backslash\{p\}) \cap U$. Como $(X, \tau)$ não 
possui pontos isolados, concluímos que $U=\{p, q\}$. Como $(X, \tau)$ também é $T_{0}$, temos que existe um aberto $W$ que contém apenas um dos pontos $p$ e q. Logo teríamos que $|U \cap W|=1$, o que contraria o fato de que $(X, \tau)$ é denso-em-si-mesmo. Portanto $(X, \tau)$ é $T_{1}$, como queríamos.

Seja $D \subset X$ um subconjunto denso. Suponha que $D \notin \tau$. Procedendo como no argumento anterior, obtemos que existe um aberto $V \in \tau$ e um ponto $x \in X$ tal que $D \cap V=\{x\}$. Porém, como $(X, \tau)$ é $T_{1},\{x\}$ é fechado, $\operatorname{logo} V \backslash\{x\}$ é aberto. Temos assim que $(V \backslash\{x\}) \cap D=\emptyset$, o que contraria a hipótese de que $D$ é denso. Logo $(X, \tau)$ é submaximal, como queríamos.

O exemplo a seguir mostra como o produto de Tychonoff não "conversa bem" com espaços discretamente gerados.

Exemplo 2.3.5 (Dow, Tkachenko, Tkachuk, Wilson [DTTW02]). Considere o espaço $2=\{0,1\}$ com a topologia discreta, e seja $2^{\mathfrak{c}}$ com o produto Tychonoff. O espaço 2 é discretamente gerado, porém o espaço $2^{\mathfrak{c}}$ não o é.

Demonstração. Vamos construir um subespaço de $2^{\mathfrak{c}}$ o qual não é discretamente gerado, logo pela proposição 2.3.2, $2^{\mathfrak{c}}$ também não o é. Van Douwen construiu em [vD93] um exemplo de um espaço Hausdorff maximal (definição 2.3.3) de cardinalidade enumerável, utilizando o Lema de Zorn. Denominaremos tal espaço por $V$, e sua topologia, a qual é maximal, por $\tau_{V}$. Seja $x \in V$ um ponto qualquer, e seja $A=V \backslash\{x\}$. Note que $x$ não pode estar no fecho de nenhum subconjunto discreto de $A$, pois todo subespaço discreto de $V$ é fechado. De fato, dado $D \subset V$ discreto, temos que $V \backslash D$ é denso, pois se existisse um aberto não vazio $U \subset D$, este geraria algum ponto isolado, e portanto $V \backslash D$ é aberto, pela proposição 2.3.4. Concluímos então que $V$ não é discretamente gerado.

Podemos construir uma cópia homeomorfa a $V$ em $2^{\mathfrak{c}}$. Para tal fim, notemos primeiramente que $V$ é zero dimensional. De fato, como $V$ é enumerável, temos em particular que $V$ é Lindelöf. Como $V$ é Lindelöf e Hausdorff, temos que $V$ é Tychonoff. Sendo assim, dado qualquer aberto $U \subset V$ e qualquer ponto $p \in U$, temos uma função contínua $f: V \longrightarrow \mathbb{I}$ tal que $f(p)=0$ e $f(y)=1$ para todo $y \in V \backslash U$. Porém , como $V$ é enumerável, $f$ não pode ser sobrejetora. Podemos então escolher $0<r<1$ tal que $r$ não está na 
imagem da função $f$. Temos então que o conjunto $W=f^{-1}([0, r[) \subset U$ é um aberto graças à continuidade de $f$. Além disso, temos também que $\left.\left.f^{-1}(] r, 1\right]\right)=V \backslash W$, portanto $W$ é também fechado. O conjunto $\mathcal{B}$ formado por todos os $W$ assim construídos é uma base de $V$ que testemunha a zero dimensionalidade de $V$.

A partir de tal base $\mathcal{B}$ de abertos-fechados, vamos construir o subespaço de $2^{\mathfrak{c}}$ que procuramos. Como $|\mathcal{P}(V)|=\mathfrak{c}$, existe uma bijeção $\varphi: \mathcal{P}(V) \longrightarrow \mathfrak{c}$. Construiremos então uma função $\psi: V \longrightarrow 2^{\mathfrak{c}}$ definida para cada $p \in V$ e cada $S \subset V$ por:

$$
\psi_{\varphi(S)}(p)= \begin{cases}1 & \text { se } p \in S \in \mathcal{B} \\ 0 & \text { caso contrário }\end{cases}
$$

Note que o fato de que $V$ é $T_{1}$ garante a injetividade de $\psi$, pois para cada ponto $p \in V, \psi(p)$ é o único ponto na imagem de $\psi$ que assume o valor 0 na coordenada $\varphi(V \backslash\{p\})$.

Vamos mostrar que $\psi$ é contínua. Dado $j \in I$, seja $V_{j}(1)=\prod_{i \in \mathfrak{c}} A_{i, j}$, onde $A_{i, j}=2=\{0,1\}$ para $i \neq j$ e $A_{i, j}=\{1\}$ para $i=j$. Seja também $V_{j}(1)=\prod_{i \in \mathfrak{c}} B_{i, j}$, onde $B_{i, j}=2$ para $i \neq j$ e $B_{i, j}=\{0\}$, para $i=j$. Como estamos trabalhando com o produto de Tychonoff, temos que todos os abertos de $2^{\mathfrak{c}}$ são intersecções de uma quantidade finita de abertos do tipo $V_{j}(n)$, com $j \in \mathfrak{c}, n \in\{0,1\}$. Caso $\varphi^{-1}(j) \notin \mathcal{B}$, temos que $\psi^{-1}\left(V_{j}(0)\right)=V$ e $\psi^{-1}\left(V_{j}(1)\right)=\emptyset$, ambos abertos. Já no caso em que $\varphi^{-1}(j) \in \mathcal{B}$, temos que $\psi^{-1}\left(V_{j}(1)\right)=\varphi^{-1}(j)$ e $\psi^{-1}\left(V_{j}(0)\right)=V \backslash \varphi^{-1}(j)$, sendo que ambos são abertos, pois $\mathcal{B}$ é formada por abertos-fechados.

Por fim, vamos mostrar que $\psi$ é aberta sobre a sua imagem. Dado um aberto $A \subset V$, pela definição de base, podemos encontrar $\mathcal{B}^{\prime} \subset \mathcal{B}$ tal que $A=\bigcup \mathcal{B}^{\prime}$. Temos então que

$$
\psi(A)=\psi\left(\bigcup \mathcal{B}^{\prime}\right)=\bigcup_{B \in \mathcal{B}^{\prime}}(\psi(B))=\bigcup_{B \in \mathcal{B}^{\prime}}\left(\psi(V) \cap V_{\varphi(B)}(1)\right)
$$

Logo $\psi(A)$ é aberto em $\psi(V)$, como queríamos

Pela proposição 2.3.2, concluímos que $2^{\mathfrak{c}}$ não é discretamente gerado.

Tal resultado ainda pode ser melhorado. Admitindo a existência de um 
L-espaço, foi demonstrado em [DTTW02] que mesmo sem a hipótese do contínuo o espaço $2^{\omega_{1}}$ não é discretamente gerado. Atualmente já temos um exemplo de um $L$-espaço em ZFC [Moo06], portanto um produto de Tychonoff de $\aleph_{1}$ espaços discretamente gerados já é o suficiente para se perder tal propriedade. Como vimos, tal propriedade é hereditária, logo dado um produto de Tychonoff de uma quantidade não enumerável de espaços topológicos quaisquer, basta encontrarmos em cada fator uma cópia isomorfa de 2 para que o produto não seja discretamente gerado.

Teorema 2.3.6 (Tkachuk, Wilson [TW12]). Para cada $i \in I$, seja $X_{i}$ um espaço topológico monotonamente normal (definição 1.2.12). Então o produto caixa $\square_{i \in I} X_{i}$ é discretamente gerado.

Demonstração. Seja $A \subset \square_{i \in I} X_{i}$, e seja $z \in \bar{A}$. Para cada $a \in A$, vamos usar a regularidade de cada $X_{i}$, com $i \in I$, para construir uma vizinhança aberta $U(a)=\prod_{i \in I} U_{i}(a)$ de $a$ de maneira que, para todo $i \in I$ tal que $z_{i} \neq a_{i}$, temos $z_{i} \notin \overline{U_{i}(a)}$. Sejam também $\kappa=\chi\left(z, \square_{i \in I} X_{i}\right)$ e $\mathcal{G}=\left\{G_{\alpha}: \alpha \in \kappa\right\}$ uma base local para $z$.

Por indução, vamos construir as sequências de pontos $a(\alpha) \in A$ e de abertos básicos $V(\alpha) \subset \square_{i \in I} X_{i}$, ambas de comprimento menor ou igual a $\kappa$. Começamos escolhendo algum $a(0) \in A \cap G_{0}$, e então definimos $V(0)=$ $U(a(0))$. Fixemos então um $\alpha<\kappa$ e suponhamos $a(\beta)$ e $V(\beta)$ já construídos, para todo $\beta<\alpha$, satisfazendo as seguintes condições:

1. $a(\beta) \in A \cap G_{\beta}$

2. $a(\beta) \in V(\beta) \subset U\left(a_{\beta}\right)$

3. A família $\left\{W_{b}: \beta<\alpha\right\}$, onde $W_{\beta}=\prod_{i \in I} \mu\left(a_{i}(\beta), V_{i}(\beta)\right)$, é dois a dois disjunta.

Para prosseguir, vamos precisar de uma consequência da condição 3. Note que tal condição garante que o conjunto $D_{\alpha}=\{a(\beta): \beta<\alpha\}$ seja discreto. Temos então:

$$
z \in \overline{D_{\alpha}} \Longleftrightarrow z \in \overline{\bigcup_{\beta<\alpha} W_{\beta}}
$$


Para provar tal afirmação, suponhamos que $z \in \overline{\bigcup_{\beta<\alpha} W_{\beta}} \backslash \overline{D_{\alpha}}$. De $z \notin$ $\overline{D_{\alpha}}$, obtemos uma vizinhança aberta $H=\prod_{i \in I} H_{i}$ de $z$ tal que $H \cap D_{\alpha}=\emptyset$. Por outro lado, definindo $W=\prod_{i \in I} \mu\left(z_{i}, H_{i}\right), z \in \overline{\bigcup_{\beta<\alpha} W_{\beta}}$ garante que $W$ intersecciona $\bigcup_{\beta<\alpha} W_{\beta}$, logo existe algum $\beta<\alpha$ tal que $W \cap W_{\beta} \neq \emptyset$. Portanto, para todo $i \in I$, temos $\mu\left(z_{i}, H_{i}\right) \cap \mu\left(a_{i}(\beta), V_{i}(\beta)\right) \neq \emptyset$. Dado $i \in I$, se $z_{i}=a_{i}(\beta)$, então $a_{i}(\beta) \in H_{i}$. Se, ao invés disso, tivermos $a_{i}(\beta) \neq x_{i}$, por construção temos que $z_{i} \notin U_{i}(a(\beta))$, $\operatorname{logo} z \notin V_{i}(\beta)$, pela condição 2. Como $X_{i}$ é monotonicamente normal, temos neste caso também que $a_{i}(\beta) \in H_{i}$. Logo $a(\beta) \in H \cap D_{\alpha}$, o que é um absurdo. Isto conclui um lado da afirmação, enquanto que o outro vem do fato de que $\overline{D_{\alpha}} \subset \overline{\bigcup_{\beta<\alpha} W_{\beta}}$.

Se por um acaso tivermos $z \in \overline{D_{\alpha}}$, não é necessário prosseguir com a indução, pois o teorema já estaria provado. Suponhamos então que $z \notin \overline{D_{\alpha}}$. Pela afirmação demonstrada no parágrafo anterior, temos que $z \notin \overline{\bigcup_{\beta<\alpha} W_{\beta}}$. Portanto, existe algum aberto básico $E$ tal que $z \in E$ e $E \cap\left(\bigcup_{\beta<\alpha} W_{\beta}\right)=\emptyset$. Definimos então $a(\alpha)$ como um ponto qualquer de $E \cap G_{\alpha} \cap A$ e $V(\alpha)$ tal que $a(\alpha) \in V(\alpha) \subset E \cap U(a(\alpha))$. Temos então que para todo $\beta<\alpha+1$, as condições de 1 a 3 são satisfeitas, concluindo a indução.

Se para todo $\alpha<\kappa$ tivermos $z \notin \overline{D_{\alpha}}$, consideramos $D=\left\{a_{\alpha}: \alpha<\kappa\right\}$. Novamente a condição 3 garante que $D$ é discreto. Como $\left\{G_{\alpha}: \alpha<\kappa\right\}$ é uma base local de $z$, a condição 1 garante que $z \in D$, completando a demonstração.

Corolário 2.3.7. O produto caixa de $\kappa$ cópias da reta real $\mathbb{R}$ (ou seja, $\square^{\kappa} \mathbb{R}$ ) é discretamente gerada, para qualquer cardinal $\kappa$.

\subsection{O espaço de funções contínuas}

Dados $X, Y$ espaços topológicos, denotamos por $C(X, Y)$ o conjunto das funções contínuas de $X$ em $Y$. No caso em que $Y=\mathbb{R}$, denotamos $C(X, \mathbb{R})$ por $C(X)$.

Habitualmente, ao espaço $C(X)$ é atribuída a topologia da convergência pontual, ou seja, a topologia na qual uma sequência de funções $\left\{f_{n}: X \longrightarrow\right.$ $\mathbb{R}: n \in \omega\}$ converge para uma função $f: X \longrightarrow \mathbb{R}$ se e somente se para 
cada ponto $x \in X$, a sequência $\left\{f_{n}(x): n \in \omega\right\}$ converge para $f(x)$. Tal topologia está associada ao produto de Tychonoff.

Se, ao invés do produto de Tychonoff, utilizarmos o produto caixa, obteremos um outro espaço topológico completamente distinto. Distinto ao ponto de que não precisamos de muitas hipóteses para que o espaço obtido de tal maneira seja discreto.

Definição 2.4.1. Seja $X$ um espaço topológico, e seja $\mathbb{R}^{X}$ o conjunto de todas as funções de $X$ em $\mathbb{R}$. Denotaremos $C(X)=\left\{f \in \mathbb{R}^{X}\right.$ : fé contínua $\}$.

- Se equiparmos o conjunto $\mathbb{R}^{X}=\prod^{X} \mathbb{R}$ com a topologia de Tychonoff $e$ consideramos o conjunto $C(X)$ com a topologia de subespaço, denotamos tal espaço topológico por $C_{p}(X)$ (neste caso, $p$ indica a convergência pontual, pois nesta topologia, o conceito de convergência equivale à convergência pontual de funções).

- Se, ao invés, equiparmos $\prod^{X} \mathbb{R}$ com a topologia da caixa, denotamos por $C_{\square}(X)$ o espaço topológico formado por $C(X)$ com a topologia herdada por $\square^{X} \mathbb{R}$.

O espaço $C_{p}(X)$ deu origem a toda uma área de estudos, a $C_{p}$ teoria. Como referência no assunto, citamos [Tka11]. Já o espaço $C_{\square}(X)$ difere drasticamente de $C_{p}(X)$, de maneira que o estudo sobre ele é muito menor, e com diferentes objetivos. A primeira grande diferença é que, enquanto o espaço $C_{p}(X)$ costuma ser um subespaço denso de $\mathbb{R}^{X}$, o espaço $C_{\square}(X)$ é sempre um subespaço fechado de $\mathbb{R}^{X}$.

Proposição 2.4.2. Seja X um espaço topológico completamente regular. Considerando $\mathbb{R}^{X}$ com a topologia de Tychonoff, $C_{p}(X)$ é denso em $\mathbb{R}^{X}$.

Demonstração. Seja $U=\prod_{x \in X} U_{x} \neq \emptyset$ um aberto básico de $\mathbb{R}^{X}$. Vamos construir uma função contínua $f: X \longrightarrow \mathbb{R}$ tal que $f \in U$. Como estamos trabalhando com o produto de Tychonoff, existem finitos pontos $x_{1}, \ldots, x_{n} \in X$ tais que $U_{x}=\mathbb{R}$ para todo $x \in X \backslash\left\{x_{i}: 1 \leq i \leq n\right\}$. Como $X$ é completamente regular, em particular $X$ é Hausdorff. Portanto, podemos construir abertos $V_{1}, \ldots, V_{n}$ disjuntos tais que $x_{i} \in V_{i}$ para cada $1 \leq i \leq n$. 
Fixemos então $1 \leq i \leq n$. Primeiramente, escolhemos $r_{i} \in U_{x_{i}}$ qualquer. Em seguida, como $X$ é completamente regular, podemos construir uma função contínua $f_{i}: X \longrightarrow \mathbb{I}$ tal que $f_{i}\left(x_{i}\right)=1$ e $f_{i}(y)=0$ para cada $y \in X \backslash V_{i}$. Aplicando o mesmo procedimento para cada $1 \leq i \leq n$, definimos a função $f$ que procuramos por $f(x)=\sum_{i=1}^{n} r_{i} f_{i}(x)$.

Proposição 2.4.3. Seja $X$ um espaço topológico qualquer. Então $C_{\square}(X)$ é um subconjunto fechado de $\square^{X} \mathbb{R}$.

Demonstração. Considere $f \in \mathbb{R}^{X} \backslash C(X)$. Então, existe um ponto $x_{0} \in X$ no qual $f$ é descontínua. Por definição, existe então uma vizinhança $U$ de $f\left(x_{0}\right)$ tal que para cada vizinhança $V$ de $x_{0}$ existe um $x_{V} \in V$ tal que $f\left(x_{V}\right) \notin \bar{U}$. Desta forma, para cada $V$ podemos encontrar uma vizinhança aberta $W_{V}$ de $f\left(x_{V}\right)$ tal que $W_{V} \cap U=\emptyset$. Definimos então $U_{x_{0}}=U, U_{x_{V}}=W_{V}$ para cada vizinhança aberta $V$ de $x_{0}$, e $U_{x}=\mathbb{R}$ para os demais $x \in X$. Dessa forma, temos $f \in \prod_{x \in X} U_{x} \subset \mathbb{R}^{X} \backslash C(X)$.

Mais do que apenas ser sempre fechado, a principal característica do espaço $C_{\square}(X)$ é a sua propensão a ser discreto.

Definição 2.4.4. Dizemos que um espaço topológico $X$ é $C_{\square}$-discreto se o espaço $C_{\square}(X)$ for discreto.

Tal conceito foi introduzido por Tamariz-Mascarúa e Villegas-Rodríguez em [TV02], onde são investigadas as relações entre os espaços $C_{\square}$-discretos e os espaços quase-w-resolúveis (definição 2.4.6, item 2.4.6). Além desta, precisaremos de algumas outras definições.

Observação 2.4.5. A menos de menção contrária, todos os resultados e definições até o final desta seção foram retirados de [TV02].

\section{Definição 2.4.6.}

- Seja $\kappa$ um cardinal. Dizemos que um espaço topológico X é $\kappa$-resolúvel se $X$ pode ser escrito como união disjunta de $\kappa$ subconjuntos densos. Dizemos que $X$ é resolúvel quando $X$ for 2-resolúvel. 
- Dizemos que um espaço $X$ é quase resolvível se $X$ puder ser escrito como união enumerável de conjuntos de interior vazio.

- Em particular, dizemos que $X$ é quase- $\omega$-resolúvel se $X$ for quase resolúvel e a família enumerável de conjuntos de interior vazio for crescente em relação à inclusão, isto é, $X=\bigcup_{n \in \omega} Y_{n}$, e para cada $n \in \omega$, $Y_{n} \subset Y_{n+1}$ e $Y_{n}$ tem interior vazio.

Proposição 2.4.7. Um espaço topológico $X$ é quase-w-resolúvel se e somente se existe uma partição $P=\left\{X_{n}: n \in \omega\right\}$ de $X$ tal que cada aberto não vazio de $X$ intersecciona infinitos elementos de $P$.

Demonstração. Suponha $X$ um espaço quase- $\omega$-resolúvel, e seja $X=\bigcup_{n \in \omega} Y_{n}$, como na definição 2.4.6. Então a partição que procuramos é $P=\left\{Y_{0}\right\} \cup$ $\left\{Y_{n+1} \backslash\left(\bigcup_{0 \leq i \leq n} Y_{i}\right): n \in \omega\right\}$. De fato, seja $V \neq \emptyset$ um aberto em $X$; se $A$ interceptasse uma quantidade finita de elementos de $P$, teríamos $A \subset X_{m}$, para algum $m$ suficientemente grande, o que contraria o fato de que $X_{m}$ tem interior vazio. $\operatorname{Logo} A$ intersecciona infinitos elementos de $P$, como gostaríamos.

Por outro lado, seja $P=\left\{X_{n}: n \in \omega\right\}$ uma partição de $X$ como no enunciado da proposição. Então podemos escrever $X=\bigcup_{n \in \omega} Y_{n} \operatorname{com} Y_{n}=$ $\bigcup_{0 \leq i \leq n} X_{i}$, satisfazendo as condições da definição 2.4.6. De fato, fixado $n \in \omega$, seja $A \subset Y_{n}$ um aberto. Supondo por absurdo $A \neq \emptyset$, então $A$ intersecciona infinitos elementos de $P$, o que contraria a hipótese de que $A \subset Y_{n}$. Portanto $Y_{n}$ tem interior vazio, como queríamos.

O resultado mais imediato sobre espaços $C_{\square}$-discreto é que eles precisam ao menos ser infinitos, como mostra a proposição a seguir.

Proposição 2.4.8. Um espaço finito não é $C_{\square}$-discreto.

Demonstração. Seja $X$ espaço topológico com $|X|=n<\omega$. Consideremos $f: X \longrightarrow \mathbb{R}$ a função constante igual a 0 . Seja $V$ uma vizinhança de $f \mathrm{em}$ $\square^{X} \mathbb{R}$ do tipo $V=\prod_{i \leq n} V_{i}$, com $\left.V_{i}=\right]-a_{i}, a_{i}$ [ para cada $i \leq n$. Considere $g: X \longrightarrow \mathbb{R}$ a função constante igual a $\frac{\min _{i \leq n} a_{i}}{2}$. Temos que $g \in V \cap C(X)$, portanto $f$ não é ponto isolado de $C_{\square}(X)$, como queríamos. 
Outra condição necessária básica para um espaço $X$ ser $C_{\square}$-discreto é que, caso $X$ seja $T_{1}$, então $X$ precisa ser denso-em-si-mesmo.

Proposição 2.4.9. Se $X$ é um espaço topológico $T_{1}$ e $C_{\square \text {-discreto, então } X}$ é denso-em-si-mesmo.

Demonstração. Seja $X$ um espaço topológico $T_{1}$ com infinitos pontos, e suponha que $x \in X$ seja um ponto isolado. Como $X$ é $T_{1}$, o conjunto $\{x\}$ é aberto fechado. Assim, podemos escrever $X=\{x\} \oplus(X \backslash\{x\})$, onde $\oplus$ indica a soma topológica ${ }^{1}$. Podemos escrever de tal forma pois, como $\{x\}$ e $X \backslash\{x\}$ são ambos abertos, em ambos os casos a topologia de subespaço coincide com a restrição da topologia ao subconjunto.

Como $X=\{x\} \oplus(X \backslash\{x\})$, então $C_{\square}(X)=C_{\square}(\{x\}) \oplus C_{\square}(X \backslash\{x\})=$ $\mathbb{R} \times C_{\square}(X)$, o qual não é discreto.

Por outro lado, uma condição suficiente para um espaço com infinitos pontos ser $C_{\square}$-discreto é que as únicas funções contínuas de $X$ em $\mathbb{R}$ sejam as funções constantes.

Proposição 2.4.10. Seja $X$ um espaço topológico, $|X| \geq \aleph_{0}$. Se as únicas funções contínuas de $X$ em $\mathbb{R}$ forem as constantes, então $X$ é $C_{\square}$-discreto.

Demonstração. Fixemos $f \in C(X)$, e seja $r \in \mathbb{R}$ o único valor na imagem de $f$. Como $X$ possui infinitos pontos, podemos escolher uma sequência $\left(x_{n}\right)_{n \in \omega}$ de pontos distintos de $X$. Para cada $n \in \omega$, definimos $\left.A_{x_{n}}=\right] r-1 / n, r+1 / n[$. Para os demais $x \in X$, definimos $A_{x}=\mathbb{R}$. Dessa forma, temos que $C(X) \cap$ $\prod_{x \in X} A_{x}=\{f\}, \operatorname{logo} f$ é ponto isolado de $C(X)$, como queríamos.

Observação 2.4.11. Note que na proposição 2.4.9, a hipótese do espaço ser $T_{1}$ não pode ser diminuída. Considere um conjunto $X$ com infinitos pontos, e seja $a \in X$ um ponto qualquer. Seja $\tau$ a topologia sobre $X$ dada por $\{A \subset X: a \in A\}$. Temos então que $(X, \tau)$ é $T_{0}$, possui um ponto isolado porém é $C_{\square}$-discreto pela proposição 2.4.10.

\footnotetext{
${ }^{1}$ União disjunta equipada com a topologia gerada pela união das topologias dos somandos.
} 
O motivo para introduzirmos neste texto o conceito de quase- $\omega$-resolvibilidade é, como comentamos, sua íntima relação com o conceito de $C_{\square}$-discrição. Mais especificamente, o primeiro implica no segundo. Além disso, para espaços $T_{3 \frac{1}{2}}$, tais conceitos são equivalentes.

Teorema 2.4.12. Seja $X$ um espaço quase-w-resolúvel. Então $X$ é $C_{\square^{-}}$ discreto.

Demonstração. Seja $P=\left\{X_{n}: n \in \omega\right\}$ uma partição de $X$ como na proposição 2.4.7. Fixemos algum $f \in C(X)$. Para cada $n \in \omega$ e para cada $x \in X_{n}$, definimos $\left.G_{x}=\right] f(x)-1 / n, f(x)+1 / n[$. Temos então a caixa aberta $G=\prod_{x \in X} G_{x}$. Pela construção, temos que $f \in G$. Vamos mostrar que $G \cap C(X)=\{f\}$. Seja $g \in G \cap C(X)$. Fixemos então $x_{0} \in X$ e $n_{0} \in \omega$. Como $f$ e $g$ são contínuas em $x_{0}$, podemos encontrar $V$ uma vizinhança aberta de $x_{0}$ em $X$ que satisfaça simultaneamente $\left.f(V) \subset\right] f\left(x_{0}\right)-1 / n_{0}, f\left(x_{0}\right)+1 / n_{0}[$ e $g(V) \subset] g\left(x_{0}\right)-1 / n_{0}, g\left(x_{0}\right)+1 / n_{0}$ [. Como todo aberto não vazio de $X$ intersecciona infinitos elementos de $P$, podemos escolher $m_{0}>n_{0}$ tal que $V \cap X_{m_{0}} \neq \emptyset$. Seja então $y \in V \cap X_{m_{0}}$, pela desigualdade triangular obtemos:

$$
\begin{aligned}
d\left(f\left(x_{0}\right), g\left(x_{0}\right)\right) & \leq d\left(f\left(x_{0}\right), f(y)\right)+d\left(f(y), g\left(x_{0}\right)\right) \\
& \leq d\left(f\left(x_{0}\right), f(y)\right)+d(f(y), g(y))+d\left(g(y), g\left(x_{0}\right)\right) \\
& \leq \frac{1}{n_{0}}+\frac{1}{m_{0}}+\frac{1}{n_{0}} \\
& \leq \frac{3}{n_{0}}
\end{aligned}
$$

Assim, variando $n_{0}$ para cada $x_{0}$ fixado, concluímos que $f=g$ e, portanto, $G \cap C(X)=\{f\}$, como queríamos.

Teorema 2.4.13. Seja $X$ um espaço $T_{3 \frac{1}{2}}$. Então $X$ é quase- $\omega$-resolúvel se e somente se $X$ é $C_{\square}$-discreto.

Demonstração. Seja $X$ um espaço $T_{3 \frac{1}{2}}$. Pelo teorema 2.4.12, falta apenas mostrar que se $X$ é $C_{\square}$-discreto, então $X$ é quase-w-resolúvel. Supondo $X$ $C_{\square}$-discreto, podemos encontrar uma caixa aberta $G=\prod_{x \in X} G_{x}$ tal que $G \cap C(X)=\{f\}$, onde $f$ é a função constante igual a zero. Definimos então 
uma função $d: X \longrightarrow \omega$ dada por $d(x)=\min \{n \in \omega:]-1 / n, 1 / n\left[\subset G_{x}\right\}$ para cada $x \in X$.

A partir de $d$, definimos $Y_{n}=\{x \in X: d(x) \leq n+1\}$ para cada $n \in \omega$. Pela construção de $d$, é fácil ver que $Y_{n} \subset Y_{n+1}$ para cada $n \in \omega$. Portanto, para que $X$ seja quase- $\omega$-resolúvel, basta mostrar que, para todo $n \in \omega, Y_{n}$ tem interior vazio.

Suponha que para algum $n_{0}>0$ exista um aberto não vazio $A$ tal que $A \subset Y_{n_{0}}$. Fixando $x_{0} \in A$, podemos construir uma função contínua $g: X \longrightarrow$ $\mathbb{R}$ tal que $g\left(x_{0}\right)=\frac{1}{2 n_{0}}$ e $g(y)=0$ para todo $y \in X \backslash A$, pois $X$ é $T_{3 \frac{1}{2}}$. Temos que $g \neq f$, e vamos mostrar que $g \in G$.

Primeiramente, se $x \in X \backslash A$, temos $h(x)=0 \in G_{x}$. Caso contrário, temos $x \in A \subset Y_{n_{0}}$, logo pela definição de $Y_{n_{0}}$, temos $d(x) \leq n_{0}+1$ e, portanto, ]$\frac{-1}{n_{0}+1}, \frac{1}{n_{0}+1}\left[\subset G_{x}\right.$. Já pela construção de $g$ temos que $g(x) \leq \frac{1}{2 n_{0}} \leq \frac{1}{n_{0}+1}$, do que concluímos que $g(x) \in G_{x}$, como queríamos.

Temos então que $g \in C(X) \cap G$, o que contraria a escolha de $G$. Logo $Y_{n}$ tem conteúdo vazio para todo $n \in \omega$, e portanto $X$ é quase- $\omega$-resolúvel.

Estabelecida a relação entre a quase- $\omega$-resolubilidade e a $C_{\square}$-discrição, vamos estudar algumas propriedades para determinar se um espaço é quase- $\omega$ resolúvel. Assim, a próxima proposição apresenta condições suficientes para uma função sobrejetora $f$ entre dois espaços topológicos refletir ou preservar a quase- $\omega$-resolubilidade.

Proposição 2.4.14. Sejam $X, Y$ espaços topológicos e $f: X \longrightarrow Y$ sobrejetora.

1. Se $Y$ for quase-w-resolúvel e a imagem por $f$ de todo aberto não vazio de $X$ tiver interior não vazio, então $X$ é quase- $\omega$-resolúvel.

2. Se $X$ for quase- $\omega$-resolúvel e $f$ for continua e injetora, então $Y$ é quase$\omega$-resolúvel.

A demonstração dos itens 1 e 2, consiste basicamente em verificar que a pré-imagem e a imagem de uma partição quase- $\omega$-resolúvel, sob as respectivas hipóteses, também é uma partição quase- $\omega$-resolúvel, o que pode ser feito diretamente da definição. 
Definição 2.4.15. Um espaço topológico $X$ é dito $\sigma$-discreto se $X$ puder ser escrito como união enumerável de subespaços discretos.

Proposição 2.4.16. Seja $X$ um espaço denso-em-si-mesmo. Se $X$ é $T_{0}$ e $\sigma$-discreto, então $X$ é quase- $\omega$-resolúvel.

Demonstração. Seja $X$ como na hipótese. Então podemos escrever $X=$ $\bigcup_{n \in \omega} X_{n}$, onde, para cada $n \in \omega, X_{n}$ é discreto e tais que $X_{n} \neq X_{m}$ sempre que $n \neq m$. Podemos supor isso, pois caso $X$ fosse união finita de subespaços discretos, como $X$ é $T_{0}$, poderíamos construir um ponto isolado em $X$.

Construímos então uma sequência $\mathcal{Z}=\left(Z_{n}\right)_{n \in \omega}$ dada por $Z_{n}=X_{n} \backslash$ $\bigcup_{i<n} X_{i}$ para cada $n \in \omega$. Temos assim que cada $Z_{n}$ é um subespaço discreto de $X$. Além disso, temos que para cada $n \in \omega$, existe um $n_{0}>n$ tal que $Z_{n_{0}} \neq \emptyset$, pois caso contrário, $X$ seria escrito como união finita de subespaços discretos. Dessa forma, excluindo os elementos vazios da sequência, obtemos uma subsequência $\mathcal{Z}^{\prime} \subset \mathcal{Z}$, a qual forma uma partição de $X$ como na proposição 2.4.7. De fato, todo aberto não vazio de $X$ intercepta infinitos elementos de $\mathcal{Z}$. Suponha que um aberto $A \subset X$ intercepte finitos elementos de $\mathcal{Z}$. Como $A$ é aberto, poderíamos escrever $A$ como uma união finita de subespaços discretos em $A$. Como $A$ herda $T_{0}$ de $X$, concluiríamos que existe um ponto isolado de $A$. Porém como $A$ é aberto, tal ponto também seria ponto isolado de $X$, o que seria um absurdo.

Vamos introduzir agora alguns conceitos relacionados aos pontos isolados.

Definição 2.4.17. Seja $(X, \tau)$ um espaço topológico. Definimos o caráter de dispersão de $(X, \tau)$ por $\Delta(X)=\min \{|U|: U \in \tau \backslash\{\emptyset\}\}$.

Note que esta função cardinal indica o quão longe um espaço está de possuir pontos isolados.

O próximo lema é um pedaço de um resultado em $\left[\mathrm{AST}^{+} 00\right]$.

Lema 2.4.18 ( $\left.\left[\mathrm{AST}^{+} 00\right]\right)$. Suponha que exista um espaço submaximal $X$ o qual não é $\sigma$-discreto. Então existe $Y$ um espaço topológico de Baire não resolúvel denso-em-si-mesmo. 
Demonstração. Seja $X$ um espaço submaximal não $\sigma$-discreto. Definimos $S=\{U \subset X: U$ é aberto não vazio e $\sigma$-discreto $\}$, e consideramos $Y=\bar{\bigcup} S$.

Vamos mostrar que $Y$ é $\sigma$-discreto. Para cada $U \in S$, podemos escrever $U=\bigcup_{n \in \omega} U_{n}$, com $U_{n}$ subespaço discreto de $U$ para cada $n \in \omega$. Como cada $U \in S$ é aberto, temos que $U_{n}$ é subespaço discreto também segundo a topologia de subespaço herdada de $X$, e da mesma forma de $\bigcup S$. Definimos então $S_{n}=\bigcup\left\{U_{n}: U \in S\right\}$ para cada $n \in \omega$. Temos então que para todo $n \in \omega, S_{n}$ é um subconjunto discreto de $\bigcup S$, e portanto $\bigcup S$ é $\sigma$-discreto.

Como $X$ é submaximal, temos que qualquer conjunto de interior vazio é discreto. De fato, seja $F \subset X$ de interior vazio. Então para cada $p \in F$, temos que $(X \backslash F) \cup\{p\}$ é denso, e portanto aberto em $X$. Dessa forma, $F$ é discreto. Como $Y$ é aberto, sua fronteira $\partial Y$ tem interior vazio, logo discreta. Concluímos assim que $Y$ é $\sigma$-discreto, como queríamos.

Definimos então $Z=X \backslash Y$. Temos que $Z$ é aberto não vazio, e não contém nenhum subconjunto aberto $\sigma$-discreto. Como $Z$ é aberto, $Z$ herda de $X$ a submaximalidade, sendo assim um espaço não resolúvel e sem pontos isolados.

Por fim, vamos mostrar que $Z$ é de Baire. Seja $\left(U_{n}\right)_{n \in \omega}$ uma família de abertos densos em $Z$. Então para cada $n \in \omega$, temos que $Z \backslash U_{n}$ é um fechado discreto. Considere então o conjunto:

$$
P=Z \backslash\left(\bigcap_{n \in \omega} U_{n}\right)=\bigcup_{n \in \omega}\left(Z \backslash U_{n}\right)
$$

$P$ é então $\sigma$-discreto e, portanto, não pode conter nenhum aberto não vazio. Portanto, $\bigcap_{n \in \omega} U_{n}$ é denso, como queríamos.

Lema 2.4.19. Se $X$ é um espaço $T_{0}$ maximal não quase-w-resolúvel, então $X$ é de Baire, não resolúvel e denso-em-si-mesmo.

Demonstração. Seja $X$ um espaço $T_{0}$ maximal que não seja quase- $\omega$-resolúvel. Pela proposição 2.3.4, temos que $X$ é submaximal. Temos então que $X$ não é $\sigma$-discreto, pois caso o fosse, pela proposição 2.4.16, teríamos $X$ quase$\omega$ resolúvel. Logo, pelo lema 2.4.18, concluímos a existência de um espaço topológico de Baire não resolúvel denso-em-si-mesmo. 
Incluímos também alguns resultados que serão usados como ferramenta para os resultados na próxima subseção.

Lema 2.4.20 (Hewitt [Hew43]). Todo espaço topológico denso-em-si-mesmo pode ser escrito como a união disjunta de um subespaço resolúvel e um hereditariamente não resolúvel.

Lema 2.4.21 (Foran, Liebnitz [FL91]). Se X é um espaço denso-em-simesmo, então são equivalentes:

1. X é quase resolúvel.

2. Podemos escrever $X=X_{1} \cup X_{2}$, de forma que:

- se $X_{1} \neq \emptyset$, então $X_{1}$ é um fechado, resolúvel de interior vazio

- $X_{2}$ é um aberto magro (ou seja, $X_{2}=\bigcup_{n \in \omega} S_{n}$, tal que para todo $n \in \omega$, temos $\operatorname{int}\left(\overline{S_{n}}\right)=\emptyset$ )

3. Existe uma função $f: X \longrightarrow \omega$ tal que $f$ seja descontínua em todos os pontos de $X$.

A partir desses resultados, obtemos:

Corolário 2.4.22. Se X é um espaço de Baire não resolúvel denso-em-simesmo, então $X$ não é quase resolúvel.

Demonstração. Se $X$ é um espaço de Baire sem pontos isolados, então pelo lema 2.4.20, $X$ contém um subespaço $Y$ aberto, não vazio e hereditariamente não resolúvel. Note que $Y$ é de Baire. Se $X$ é quase resolúvel, então $Y$ também o é, porém isso implica, pelo lema 2.4.21 que $Y$ é um conjunto magro, o que é um absurdo.

\subsubsection{Cardinais mensuráveis}

Os espaços $C_{\square}$-discretos estão também associados à existência de cardinais mensuráveis. Se assumirmos a existência deles, podemos construir contra exemplos para a recíproca do teorema 2.4.12. Por outro lado, se trabalharmos em algum modelo de ZFC que não admita nenhum cardinal inacessível, 
então podemos construir um resultado que indicaria uma recíproca para a proposição 2.4.9.

Definição 2.4.23. Um cardinal não enumerável $\kappa$ é dito mensurável se $\kappa$ admite um ultrafiltro livre $\kappa$-completo (definição 1.1.4).

Observação 2.4.24. Existe uma outra definição equivalente para o conceito de cardinal mensurável envolvendo uma medida binária sobre o cardinal, o que justifica a nomenclatura. Contudo, tal definição é irrelevante para este contexto.

É possível demonstrar que os cardinais mensuráveis são fortemente inacessíveis. Este e outros resultados sobre cardinais mensuráveis podem ser encontrados em [Jec03].

Exemplo 2.4.25. Suponha a existência de um cardinal mensurável $\kappa$. Então, existe $X$ um espaço topológico $T_{0}$, Baire resolúvel, $C_{\square}$-discreto o qual não é quase- $\omega$-resolúvel e tal que $\Delta(X)=\kappa$.

Demonstração. Seja $\kappa$ um cardinal mensurável. Escolhemos então $\mathcal{U} \subset$ $\mathcal{P}(\kappa)$ um ultrafiltro livre $\kappa$-completo, como na definição 2.4.23. Defini$\operatorname{mos} X=\kappa \cup\{\mathcal{U}\}$. Definimos também a topologia $\tau$ sobre $X$ dada por $\tau=\{A \in \mathcal{P}(X): \mathcal{U} \in A$ e $A \cap \kappa \in \mathcal{U}\} \cup\{\emptyset\}$.

Primeiramente, note que $\kappa$ e $\{\mathcal{U}\}$ são densos disjuntos de $X$, o qual é, portanto, resolúvel. Além disso, temos que se $C \in U$, então $|C|=\kappa$. De fato, seja $P \subset \kappa$ tal que $|P|<\kappa$. Como $U$ é ultrafiltro, pela proposição 1.1.5, para cada $p \in P$ temos que ou $\{p\} \in U$ ou $\kappa \backslash\{p\} \in U$, do que podemos tirar que $\kappa \backslash\{p\} \in U$, pois $\mathcal{U}$ é livre. Note que podemos escrever $\kappa \backslash P=\bigcap_{p \in P}(\kappa \backslash\{p\})$. Como $U$ é $\kappa$-completo, temos que $\kappa \backslash P \in \mathcal{U}$, logo $P \notin \mathcal{U}$. A partir disso, concluímos que $\Delta(X)=\kappa$.

Vamos mostrar que $X$ não é quase- $\omega$-resolúvel. Sejam $\left(Y_{n}\right)_{n \in \omega}$ uma família crescente de conjuntos de interior vazio tal que $X=\bigcup_{n \in \omega} Y_{n}$. Escolhemos então $n_{0} \in \omega$ tal que $\mathcal{U} \in Y_{n_{0}}$ e $n_{1} \in \omega$ tal que $Y_{n_{1}} \cap \kappa \in \mathcal{U}$. Podemos escolher tal $n_{1}$ pois, caso ele não existisse, teríamos que $\kappa \backslash Y_{n} \in \mathcal{U}$ para todo $n \in \omega$, pois $\mathcal{U}$ é ultrafiltro. Isso implicaria que $\emptyset \in \mathcal{U}$, pois $\mathcal{U}$ é $\kappa$-completo. 
Escolhemos então algum $k>n_{0}, n_{1}$, e temos que $Y_{k}$ é aberto não vazio, logo possui interior não vazio.

Considere $f \in C(X)$ e $x \in X$. Como todo aberto de $X$ contém o ponto $\mathcal{U}$, temos que $f(\mathcal{U}) \in] f(x)-\epsilon, f(x)+\epsilon$ [ para todo $\epsilon>0$, do que se concluí que $f(x)=f(\mathcal{U})$. Portanto, da mesma maneira que na observação 2.4.11, as únicas funções contínuas de $X$ em $\mathbb{R}$ são as constantes, e portanto pela proposição 2.4.10, $X$ é $C_{\square}$-discreto.

Por fim, temos que $X$ é espaço de Baire: seja $\left(D_{n}\right)_{n \in \omega}$ uma sequência de abertos densos em $X$. Para cada $n \in \omega$, temos que $\mathcal{U} \in D_{n}$, $\log$ o $\mathcal{U} \in$ $\bigcap_{n \in \omega} D_{n}$, e portanto $\bigcap_{n \in \omega} D_{n}$ é denso em $X$.

Exemplo 2.4.26. Suponha a existência de um cardinal mensurável $\kappa$. Então, existe $X$ um espaço topológico $T_{0}$, Baire, submaximal, $C_{\square}$-discreto o qual não é quase resolúvel e tal que $\Delta(X)=\kappa$.

Demonstração. Seja $\kappa$ um cardinal mensurável, e considere $X$ como no exemplo anterior (exemplo 2.4.25). Diferentemente do item anterior, considere sobre $X$ a topologia $\theta=\{A \in \mathcal{P}(X): A \cap \kappa \in \mathcal{U}\} \cup\{\emptyset\}$. Da mesma maneira que no item anterior, equipando $X$ com tal topologia temos $\Delta(X)=\kappa$. Além disso, ainda temos também que $X$ é Baire: dada $\left(D_{n}\right)_{n \in \omega}$ sequência de abertos densos em $X$, temos que $D_{n} \cap \kappa \in \mathcal{U}$ para cada $n \in \omega$. Como $\mathcal{U}$ é $\kappa$-completo, temos $\bigcap_{n \in \omega}\left(D_{n} \cap \kappa\right) \in \mathcal{U}$. Como $\mathcal{U}$ é filtro, temos que $\bigcap_{n \in \omega} D_{n}$ é denso em $X$.

Vamos mostrar que $X$ é $C_{\square}$-discreto pela proposição 2.4.10. Seja $f$ : $X \longrightarrow \mathbb{R}$ função contínua, e suponha que existam $x, y \in X$ distintos tais que $f(x) \neq f(y)$. Como a reta real é Hausdorff, podemos achar dois abertos disjuntos $V_{x}$ e $V_{y}$ contendo respectivamente $f(x)$ e $f(y)$. Como $f$ é contínua, temos que $f^{-1}\left(V_{x}\right) \cap \kappa, f^{-1}\left(V_{y}\right) \cap \kappa \in \mathcal{U}$, o que é um absurdo, pois $f^{-1}\left(V_{x}\right) \cap$ $f^{-1}\left(V_{y}\right)=\emptyset$.

Também temos que $X$ não é quase resolúvel. Escrevendo $X=\bigcup_{n \in \omega} U_{n}$, da mesma maneira que no item anterior, conseguimos um $n_{0} \in \omega$ tal que $U_{n_{0}} \cap \kappa \in \mathcal{U}$. Logo $X_{n_{0}}$ é aberto não vazio em $X$, e portanto possuí interior não vazio. 
Por fim, temos que $X$ é submaximal, pois, como $\mathcal{U}$ é ultrafiltro, $D \subset X$ é denso se e somente se $D \cap \kappa \in \mathcal{U}$, e portanto todo denso é aberto.

Agora nosso objetivo consiste de construir a "recíproca" da proposição 2.4.9. Para isso, vamos precisar de um resultado (lema 2.4.27) que estabelece consequências topológicas para a não existência de cardinais mensuráveis. O caso em cardinalidade regular de tal resultado foi provado por Kunen, Szymansky e Tall em [KST86], baseados em um artigo sobre cardinais mensuráveis e ideais precipitados por Magidor [JMMP80].

Lema 2.4.27 (Kunen, Szymański, Tall [KST86]). Se $V=L$, então todo espaço denso-em-si-mesmo é quase resolúvel.

Demonstração. Como comentamos, o caso para espaços de cardinalidade regular deste teorema é a proposição 3.9 em [KST86]. Seja agora $X$ um espaço denso-em-si-mesmo tal que $|X|=\kappa$, onde $\kappa$ é um cardinal singular. Seja $\kappa^{+}$o cardinal sucessor de $\kappa$, o qual é regular. Considere o espaço $Z=\oplus_{\lambda \in \kappa}(X \times\{\lambda\})$. Note que $|Z|=\kappa \times \kappa^{+}$. Como $X$ não possui pontos isolados, $Z$ também não os possui, e portanto, assumindo $V=L$, temos que $Z$ é quase resolúvel. Podemos então escrever $Z=\bigcup_{n \in \omega} J_{n}$, com cada $J_{n}$ de interior vazio. A partir disso, podemos escrever $X=\bigcup_{n \in \omega} L_{n}$, onde $L_{n}=J_{n} \cap(X \times\{0\})$. Desta forma, $X$ é quase resolúvel, como queríamos.

Corolário 2.4.28. Se $V=L$, então todo espaço de Baire denso-em-simesmo é resolúvel.

Demonstração. Consequência do lema 2.4.27 e do corolário 2.4.22.

Teorema 2.4.29. Assumindo $Z F C$ e $V=L$, então todo espaço $T_{0}$ densoem-si-mesmo é quase-w-resolúvel (em particular, $C_{\square}$-discreto).

Demonstração. Supondo ZFC e $V=L$, temos que todo espaço topológico $T_{0}$ maximal é quase- $\omega$-resolúvel pelo corolário 2.4 .28 e pela contra contrapositiva do lema 2.4.19.

Considere então um espaço topológico $(X, \tau)$ denso-em-si-mesmo e $T_{0}$. Podemos expandir $\tau$ para uma topologia $\tau^{\prime}$ tal que $\tau \subset \tau^{\prime}$ e $\left(X, \tau^{\prime}\right)$ seja 
maximal. Sob as hipóteses, temos $\left(X, \tau^{\prime}\right)$ quase- $\omega$-resolúvel. Considere então a função identidade Id $:\left(X, \tau^{\prime}\right) \longrightarrow(X, \tau)$. Tal função é bijetora e contínua, logo pela proposição 2.4.14, temos que $(X, \tau)$ também é quase- $\omega$-resolúvel, como queríamos. 


\section{Capítulo 3}

\section{Paracompacidade de produtos caixa}

Como vimos no capítulo anterior, o produto caixa geralmente não é compacto (teorema 2.2.11). Sendo assim, uma pergunta natural seria: "podemos enfraquecer a compacidade a alguma outra propriedade que se comporte bem com o produto caixa?" . Esta pergunta levou ao estudo da preservação da paracompacidade dos produtos caixa, o que levou a diversos resultados. Desde então, a pergunta anterior mudou para "Sob quais condições sobre seus fatores o produto caixa é paracompacto?" , a qual virou o objeto central no estudo dos produtos caixa.

Na seção 3.1 apresentamos o produto nabla, um produto auxiliar que utilizaremos como ferramenta. Na seção 3.2 estudamos um resultado sob a hipótese do contínuo, enquanto na seção 3.3 estudamos resultados sob hipóteses menores. Em seguida, apresentamos alguns resultados relativos a produtos de ordinais na seção 3.4. Por fim, apresentamos um panorama do caso em que o produto é não enumerável na seção 3.5 e dos problemas em aberto na área, na seção 3.6.

Como referências centrais neste tópico citamos [Wil84], [vD80] e [Roi11]. 


\section{$3.1 \quad \mathrm{O}$ produto nabla}

Introduziremos nesta seção uma ferramenta importante para se trabalhar com produtos caixa: o produto Nabla. Com ele, podemos trabalhar com o produto caixa "a menos de um número finito de índices", como veremos na definição a seguir. Uma de suas principais utilidades é o fato de que o produto nabla de uma quantidade enumerável de espaços é um P-espaço (lema 3.1.4). Tal propriedade é normalmente usada em conjunto com o Nabla lemma (teorema 3.1.5), o qual demonstra, sob certas hipóteses, a equivalência entre a paracompacidade no produto caixa e no produto Nabla.

Definição 3.1.1 (Produto nabla). Seja I um conjunto de indices. Para cada $i \in I$, seja $X_{i}$ um espaço topológico. Seja $=^{*}$ a relação de equivalência em $\square_{i \in I} X_{i}$ dada por:

$$
x={ }^{*} y \Longleftrightarrow\left\{i \in I: x_{i} \neq y_{i}\right\} \text { é finito }
$$

Definimos então o produto nabla dos espaços $\left(X_{i}\right)_{i \in I}$ pelo espaço topológico dado por

$$
\nabla_{i \in I} X_{i}=\frac{\square_{i \in I} X_{i}}{=^{*}}
$$

equipado com a topologia quociente.

No caso do produto de espaços iguais, ou seja, $\forall i \in I, X_{i}=X$ para algum espaço topológico $X$, denotamos $\nabla_{i \in I} X_{i}=\nabla^{I} X$

Definimos também $q: \square_{i \in I} X_{i} \longrightarrow \nabla_{i \in I} X_{i}$ a projeção usual.

Observação 3.1.2. O conceito de produto nabla pode ser generalizado para um filtro qualquer da seguinte maneira: dados uma família de espaços topológicos $\left\{X_{i}: i \in I\right\}$ e um filtro $\mathfrak{F}$ em $I$, podemos construir uma relação de equivalência $\cong$ definida por $x \cong y \Leftrightarrow\left\{i \in I: x_{i}=y_{i}\right\} \in \mathfrak{F}$. Podemos então definir o produto nabla como acima, apenas substituindo a relação $={ }^{*}$ pela relação $\cong$. No entanto, como neste texto trabalharemos com produtos de uma quantidade enumerável de espaços, o filtro que nos interessará é o filtro dos conjuntos cofinitos (isto é, cujo complementar é finito), por isso usaremos apenas a definição 3.1.1. 
Mostraremos agora algumas propriedades básicas do produto nabla.

Proposição 3.1.3. Seja $\left(X_{i}\right)_{i \in \omega}$ uma família de espaços topológicos. Sejam também duas familias $\left\{A_{i}: i \in I\right\}$ e $\left\{B_{i}: i \in I\right\}$ tais que $\forall i \in I, A_{i}, B_{i} \subset$ $X_{i}$. Então

1. $q\left(\prod_{i \in I} A_{i}\right) \subset q\left(\prod_{i \in I} B_{i}\right)$ se e somente se $\left\{i \in I: A_{i} \not \subset B_{i}\right\}$ é finito .

2. se $I=\omega$, então a projeção $q: \square_{i \in \omega} X_{i} \longrightarrow \nabla_{i \in I} X_{i}$ é aberta.

3. $q\left(\prod_{i \in I} A_{i}\right)$ é aberto (ou fechado) se e somente se o conjunto dos $i \in I$ tais que $A_{i}$ não é aberto (ou fechado) é finito.

4. o produto nabla "comuta" com a topologia de subespaço, isto é, $\nabla_{i \in I} A_{i}$ é homeomorfo a $q\left(\prod_{i \in I} A_{i}\right)$ com a topologia de subespaço de $\nabla_{i \in I} X_{i}$, e tal homeomorfismo comuta com a projeção $q$

\section{Demonstração.}

1. Suponhamos que $\left\{i \in I: A_{i} \not \subset B_{i}\right\}$ seja finito. Seja $a \in \prod_{i \in I} A_{i}$. Note que $\left\{i \in I: a_{i} \notin B_{i}\right\} \subset\left\{i \in I: A_{i} \not \subset B_{i}\right\}$, o qual supomos finito. Te$\operatorname{mos}$ assim que $q(a) \in q\left(\prod_{i \in I} B_{i}\right), \operatorname{logo} q\left(\prod_{i \in I} A_{i}\right) \subset q\left(\prod_{i \in I} B_{i}\right)$.

Vamos agora demonstrar a contra-positiva da recíproca. Suponha$\operatorname{mos}\left\{i \in I: A_{i} \not \subset B_{i}\right\}$ infinito. Podemos escolher $a \in \prod_{i \in I} A_{i}$ tal que $a_{i} \in A_{i} \backslash B_{i}$ sempre que $A_{i} \not \subset B_{i}$. Temos então que $\left\{i \in I: a_{i} \notin B_{i}\right\}$ é infinito, de modo que $q(a) \notin q\left(\prod_{i \in I} A_{i}\right)$, como gostaríamos.

2. Notemos que $q^{-1}\left(q\left(\prod_{i \in \omega} A_{i}\right)\right)=\bigcup_{J \in[\omega]<\aleph_{0}} \prod_{i \in \omega} C(i, J)$, onde $C(i, J)$ $=X_{i}$ caso $i \in J$ e $C(i, J)=A_{i}$ caso $i \notin J$. Seja $\prod_{i \in I} A_{i}$ aberto em $\square_{i \in I} X_{i}$. Para cada $i \in I$, temos $A_{i}$ aberto em $X_{i}$. Assim, cada $\prod_{i \in \omega} C(i, J)$ é aberto em $\square_{i \in I} X_{i}$, de modo que, pela definição de topologia quociente, $q\left(\prod_{i \in \omega} A_{i}\right)$ é aberto em $\nabla_{i \in I} X_{i}$, como gostaríamos.

3. Suponhamos que o conjunto $O=\left\{i \in I\right.$ : $A_{i}$ não é aberto $\}$ seja finito. Seja $q(a) \in q\left(\prod_{i \in I} A_{i}\right)$. Para cada $i \notin O$ tal que $a_{i} \in A_{i}$, escolhemos $V_{i}$ aberto tal que $a_{i} \in V_{i} \subset A_{i}$. Para os demais, definimos $V_{i}=X_{i}$, e chamamos então $V=\prod_{i \in I} V_{i}$. Pelo item anterior, temos que $q(V)$ 
é uma vizinhança aberta de $q(a)$. Pelo item 1 , temos $q\left(\prod_{i \in I} V_{i}\right) \subset$ $q\left(\prod_{i \in I} A_{i}\right), \operatorname{logo} q\left(\prod_{i \in I} A_{i}\right)$ é aberto.

Da mesma maneira, suponhamos que o conjunto $C=\left\{i \in I: A_{i}\right.$ não é fechado $\}$ seja finito. Escolhemos um ponto $p \in$ $\square_{i \in I} X_{i}$ tal que $q(p) \notin q\left(\prod_{i \in I} A_{i}\right)$. Temos que $p_{i} \notin A_{i}$ para infinitos $i \in$ $\omega$. Como $C$ é finito, temos que $H=\cap\left\{i \in I: p_{i} \notin A_{i}\right.$ e $A_{i}$ é fechado $\}$ é infinito. Para cada $i \in H$, podemos encontrar uma vizinhança aberta $U_{i}$ de $p_{i}$ tal que $U_{i} \cap A_{i}=\emptyset$. Para $i \in I \backslash H$, definimos $U_{i}=X_{i}$. Assim, como $H$ é infinito, temos que $q\left(\prod_{i \in I} A_{i}\right) \cap q\left(\prod_{i \in I} U_{i}\right)=\emptyset$, como gostaríamos.

Para a recíproca, suponhamos que $O$ seja infinito. Então para cada $i \in O$, podemos encontrar $a_{i} \in A_{i}$ tal que $a_{i}$ não seja ponto interior de $A_{i}$. Para os demais, podemos escolher $a_{i} \in A_{i}$ qualquer. Vamos mostrar que $q(a)$, onde $a=\left(a_{i}\right)_{i \in I}$, não é um ponto interior de $q\left(\prod_{i \in I} A_{i}\right)$. Pelo item 2, é suficiente mostrar que qualquer vizinhança de $q(a)$ da forma $q\left(\prod_{i \in I} V_{i}\right)$, com $V_{i}$ aberto em $X_{i}$ para cada $i \in I$, contém um ponto $q(b)$ tal que $q(b) \notin q\left(\prod_{i \in I} A_{i}\right)$. Seja $q(V)$ uma tal vizinhança de $q(p)$. Temos que $a_{i} \notin V_{i}$ para apenas finitas coordenadas. Podemos então achar infinitas coordenadas $i \in \omega$ tais que $a_{i} \in V_{i}$ e $a_{i}$ não seja ponto interior de $A_{i}$. Para tais coordenadas, escolhemos $b_{i} \in V_{i} \backslash A_{i}$ qualquer. Para as demais coordenadas, definimos $b_{i}=a_{i}$. Assim, definindo $b=\left(b_{i}\right)_{i \in \omega}$, temos $q(b) \in q(V) \backslash q\left(\prod_{i \in \omega} A_{i}\right)$, portanto $q(b)$ testemunha que $q(a)$ não é ponto interior de $q\left(\prod_{i \in \omega} A_{i}\right)$ e, portanto, $q\left(\prod_{i \in \omega} A_{i}\right)$ não é aberto. Para o caso em que $C$ é infinito, procedemos da mesma maneira para encontrar um ponto $q(a) \in \overline{q\left(\prod_{i \in \omega} A_{i}\right)}$ tal que $q(a) \notin q\left(\prod_{i \in \omega} A_{i}\right)$.

4. Vamos mostrar que a função identidade é um homeomorfismo considerando tais topologias. Seja $V \subset \nabla_{i \in I} A_{i}$ aberto. Temos um aberto $V^{\prime} \subset \square_{i \in I} A_{i}$ tal que $V=q\left(V^{\prime}\right)$. Por sua vez, existe um aberto $U$ de $\square_{i \in I} X_{i}$ tal que $V^{\prime}=U \cap \prod_{i \in I} A_{i}$. Portanto $V=q(U) \cap q\left(\prod_{i \in I} A_{i}\right)$, ou seja, $V$ é aberto na topologia de subespaço. Por outro lado, seja agora $V$ um aberto de $q\left(\prod_{i \in I} A_{i}\right)$ na topologia de subespaço. Então 
existe $U$ aberto em $\nabla_{i \in I} X_{i}$ tal que $V=U \cap q\left(\prod_{i \in I} A_{i}\right)$. Por sua vez, $U=q\left(U^{\prime}\right)$, para algum aberto $U^{\prime} \subset \square_{i \in I} X_{i}$. Temos então que $V=q\left(U^{\prime} \cap \prod_{i \in I} A_{i}\right)$, completando a demonstração.

Note que uma aplicação importante do item 2 é que podemos obter uma base para o produto nabla a partir da projeção de uma base de abertos do produto caixa.

O próximo lema é uma das principais propriedades do produto nabla, e como dissemos no começo da seção, será uma ferramenta importante para estudar a paracompacidade dos produtos caixa. Note que a partir de agora trabalharemos com produtos de uma quantidade enumerável de espaços.

Lema 3.1.4 (Rudin). Seja $X_{i}$ um espaço topológico para cada $i \in \omega$. Então $\nabla_{i \in \omega} X_{i}$ é um P-espaço.

Demonstração. Considere uma família $\left\{A(i, n) \subset X_{i}: i, n \in \omega\right\}$ de abertos. Vamos mostrar que $\bigcap_{n \in \omega} q\left(\prod_{i \in \omega} A(i, n)\right)$ é aberto. Note que podemos supor sem perda de generalidade que esta família é uma sequência "decrescente em cada coordenada $i$ ", ou seja, uma sequência tal que vale $\forall n \in \omega, A(i, n+$ 1) $\subset A(i, n) \subset X_{i}$. Isto basta para provar o teorema, pois pelo item 2 da proposição 3.1 .3 , a projeção de produtos caixa de abertos forma uma base em $\nabla_{i \in \omega} X_{i}$.

Dado $p \in \square_{i \in I} X_{i}$ tal que $q(p) \in \bigcap_{n \in \omega} q\left(\prod_{i \in \omega} A(i, n)\right)$, vamos construir uma função crescente $f_{p}: \omega \longrightarrow \omega$ tal que $q(p) \in q\left(\prod_{i \in \omega} A\left(i, f_{p}(i)\right)\right)$. Esta função funcionará como uma diagonal em um argumento de diagonalização. Notemos que para cada $n \in \omega$, como $q(p) \in q\left(\prod_{i \in \omega} A(i, n)\right)$, existe um $k_{n} \in \omega$ tal que para todo $j \geq k_{n}$ temos $p_{j} \in A(j, n)$. Como $(A(i, n))_{n \in \omega}$ é decrescente, podemos supor $\left(k_{n}\right)_{n \in \omega}$ crescente. Vamos então construir $f_{p}$ por recurção em $i$ :

$$
f_{p}(i)=\left\{\begin{array}{cl}
0 & \text { se } i=0 \\
f_{p}(j) & \text { se } i=j+1 \text { e } i<k_{f_{p}(j)+1} \\
f_{p}(j)+1 & \text { se } i=j+1 \text { e } i \geq k_{f_{p}(j)+1}
\end{array}\right.
$$


Esta recursão garante que para todo $i>0$ tenhamos $k_{f_{p}(i)} \leq i$, de maneira que $p_{i} \in A\left(i, f_{p}(i)\right)$. Como $\left(k_{n}\right)_{n \in \omega}$ é crescente, temos também que $f$ não é eventualmente constante. Assim, temos que $q(p) \in q\left(\prod_{i \in \omega} A\left(i, f_{p}(i)\right)\right)$, como queríamos. Como cada $A(i, n)$ é aberto, $q\left(\prod_{i \in \omega} A\left(i, f_{p}(i)\right)\right)$ também o é. Assim, basta então provarmos que

$$
q\left(\prod_{i \in \omega} A\left(i, f_{p}(i)\right)\right) \subset \bigcap_{n \in \omega} q\left(\prod_{i \in \omega} A(i, n)\right)
$$

Precisamos mostrar que $\forall n \in \omega, q\left(\prod_{i \in \omega} A\left(i, f_{p}(i)\right)\right) \subset q\left(\prod_{i \in \omega} A(i, n)\right)$. Note que, como a sequência é decrescente em cada coordenada, vale para cada $n$ que $f_{p}(i)>n$ implica em $A\left(i, f_{p}(i)\right) \subset A(i, n)$. Assim, como $f_{p}$ é crescente, temos que $\left\{i: A\left(i, f_{p}(i)\right) \not \subset A(i, n)\right\}$ é finito, e portanto pelo item 1 deste lemma, temos a inclusão desejada, completando a demonstração.

Note que a existência de tal função $f_{p}$ está relacionado ao fato de que toda família enumerável de funções de $\omega$ em $\omega$ é limitada (no sentido da definição 3.3.2). Veremos mais sobre isso na seção 3.3.

Apresentamos agora o Nabla Lemma, que será nossa principal ferramenta para utilizarmos este produto. Esta versão do teorema, está em [Kun78].

Teorema 3.1.5 (Nabla Lemma). Para cada $i \in \omega$, seja $X_{i}$ um espaço topológico compacto. Temos então que $\square_{i \in \omega} X_{i}$ é paracompacto se e somente se $\nabla_{i \in \omega} X_{i}$ é paracompacto.

Demonstração. Vamos demonstrar que a projeção $q: \square_{i \in \omega} X_{i} \longrightarrow \nabla_{i \in \omega} X_{i}$ é uma função fechada para então utilizar o lema 1.2.29: Seja $F \subset \square_{i \in \omega} X_{i}$ fechado. Queremos mostrar que $q(F)$ é fechado em $\nabla_{i \in \omega} X_{i}$, ou equivalentemente, mostrar que $q^{-1}(q(F))$ é fechado em $\square_{i \in \omega} X_{i}$. Definindo a projeção $\pi_{\geq k}: \square_{i \in \omega} X_{i}=\square_{i<k} X_{i} \times \square_{k \geq k} X_{i} \longrightarrow \square_{k \geq k} X_{i}$, podemos escrever $q^{-1}(q(F))=\bigcup_{k \in \omega} \pi_{\geq k}^{-1}\left(\pi_{\geq k}(F)\right)$. Usando a hipótese de que para todo $i \in \omega X_{i}$ é compacto, vemos que para cada $k \in \omega, \pi_{\geq k}(F)$ é fechado.

De fato, seja $v \in\left(\square_{k \geq k} X_{i} \backslash \pi_{\geq k}(F)\right)$. Como $F$ é fechado em $\square_{i \in \omega} X_{i}$, podemos encontrar para cada $u \in \square_{i<k} X_{i}$ um aberto básico $U_{u} \times V_{u}$, com 
$U_{u} \subset \square_{i<k} X_{i}$ e $V_{u} \subset \square_{k \geq k} X_{i}$, tal que $(u, v) \in U_{u} \times V_{u}$ e $\left(U_{u} \times V_{u}\right) \cap F=\emptyset$. Assim, temos que $\left\{U_{u}: u \in \square_{i<k} X_{i}\right\}$ é uma cobertura aberta de $\square_{i<k} X_{i}$, o qual é compacto, pois o produto caixa de um número finito de termos coincide com o produto de Tychonoff. Assim, temos um conjunto finito de pontos $\Theta \subset \square_{i<k} X_{i}$ tais que $\square_{i<k} X_{i}=\bigcup_{u \in \Theta} U_{u}$. Temos então que $V=\bigcap_{u \in \Theta} V_{u}$ é uma vizinhança aberta de $v$. Além disso, para cada $b \in \pi_{\geq k}(F)$, existe $a \in \square_{i<k} X_{i}$ tal que $(a, b) \in F$, contudo existe $u \in \Theta$ tal que $a \in U_{u}$. Como $(a, b) \in F$, temos $(a, b) \notin U_{u} \times V_{u}$. Logo $b \notin V$. Concluímos assim que $\pi_{\geq k}(F)$ é fechado, como queríamos.

Com isso, vamos mostrar que o conjunto $\bigcup_{k \in \omega} \pi_{\geq k}^{-1}\left(\pi_{\geq k}(F)\right)$ é fechado. Note que, pelas leis de De Morgan, podemos escrever o seu complementar da seguinte maneira:

$$
\square_{i \in \omega} X_{i} \backslash \bigcup_{k \in \omega} \pi_{\geq k}^{-1}\left(\pi_{\geq k}(F)\right)=\bigcap_{k \in \omega} \pi_{\geq k}^{-1}\left(\square_{k \geq k} X_{i} \backslash \pi_{\geq k}(F)\right)
$$

Seja $p \in \bigcap_{k \in \omega} \pi_{\geq k}^{-1}\left(\square_{k \geq k} X_{i} \backslash \pi_{\geq k}(F)\right)$. Como mostramos que para cada $k \in \omega, \pi_{\geq k}(F)$ é fechado e $\pi_{\geq k}$ é contínua, então fixado $k \in \omega$ podemos escolher uma vizinhança aberta $V^{k}=\prod_{i \in \omega} V_{i}^{k}$ de $p$ contida em $\pi_{\geq k}^{-1}\left(\square_{k \geq k} X_{i} \backslash \pi_{\geq k}(F)\right)$ com $V_{i}^{k}$ aberto para todo $i \geq k$ e $V_{i}^{k}=X_{i}$ para todo $i<k$. Definimos $W_{i}=\bigcap_{k \leq i} V_{i}^{k}$. Note que $\prod_{i \in \omega} W_{i}=\bigcap_{k \in \omega}\left(V^{k}\right)$ e que tal aberto é uma vizinhança de $p$ que não intersepta $\bigcup_{k \in \omega} \pi_{\geq k}^{-1}\left(\pi_{\geq k}(F)\right)$, o que conclui a demonstração de que $q: \square_{i \in \omega} X_{i} \longrightarrow \nabla_{i \in \omega} X_{i}$ é fechada.

Assim, podemos aplicar o lema 1.2.29. O item 1 garante um sentido da demonstração, enquanto que o outro vem do item 2. Porém, para aplicar o item 2, precisamos demonstrar que para cada $p \in \nabla_{i \in \omega} X_{i}, q^{-1}(\{p\})$ é Lindelöf:

Fixemos um representante $z \in q^{-1}(\{p\})$. Então $q^{-1}(\{p\})=\left\{x \in \square_{i \in \omega} X_{i}\right.$ : $\left.x={ }^{*} z\right\}=\bigcup_{j \in \omega} L_{j}$, onde $L_{j}=\left\{x \in \square_{i \in \omega} X_{i}: \forall i \geq j, x_{i}=z_{i}\right\}$. Como por hipótese temos todos os $X_{i}$ 's compactos, temos que $q^{-1}(\{p\})$ é $\sigma$-compacto, portanto Lindelöf, como queríamos. 


\subsection{Uma equivalência da Hipótese do Contínuo}

Como mencionado anteriormente, o problema central relacionado aos produtos caixa diz respeito a encontrar condições sob as quais o produto caixa enumerável de espaços topológicos preserva a paracompacidade. Assumindo a Hipótese do Contínuo, temos uma condição necessária e suficiente para a paracompacidade de produtos caixa enumeráveis de espaços compactos regulares envolvendo o grau de Lindelöf (definição 1.2.16). Tal condição será apresentada no teorema 3.2.2. Em seguida, através do exemplo 3.2.3, mostraremos que o resultado do teorema 3.2.2 não é apenas consequência da Hipótese do Contínuo, e sim uma equivalência do mesmo.

Vamos precisar do seguinte lema por Kunen [Kun78]:

Lema 3.2.1. Seja $X$ um espaço paracompacto regular e $\mathcal{B}$ uma base de $X$. Então qualquer cobertura aberta $\mathcal{U}$ de $X$ possui um refinamento $\mathcal{V}$ tal que:

1. cada $V \in \mathcal{V}$ pode ser escrito na forma $V=\bigcap_{n \in \omega} B_{n}$, onde para cada $n \in \omega$ temos $B_{n} \in \mathcal{B}$ e $B_{n} \supset \overline{B_{n+1}}$;

2. $\forall W \in \mathcal{V},|\{V \in \mathcal{V}: W \cap V \neq \emptyset\}| \leq \mathfrak{c}$.

Demonstração. Seja $\mathcal{U}$ uma cobertura aberta do espaço paracompacto $X$, com uma base $\mathcal{B}$. Vamos construir uma sequência de coberturas abertas $\mathcal{V}_{n}$ de $X$ satisfazendo, para cada $n \in \omega$, as seguintes propriedades:

I $\mathcal{V}_{n+1}$ é um refinamento aberto localmente finito de $\mathcal{V}_{n}$

II Dado $V_{n+1} \in \mathcal{V}_{n+1}, V_{n+1}$ intersecciona no máximo uma quantidade finita de elementos de $\mathcal{V}_{n}$

III $\forall V_{n+1} \in \mathcal{V}_{n+1}, \exists B \in \mathcal{B}, \exists V_{n} \in \mathcal{V}_{n}, V_{n+1} \subset B \subset \bar{B} \subset V_{n}$

Começamos usando a paracompacidade de $X$ para construir $\mathcal{V}_{0}$ um refinamento aberto localmente finito de $\mathcal{U}$. Fixamos então $n \in \omega$ e supomos que $\mathcal{V}_{n}$ foi construída satisfazendo as propriedades desejadas. Para cada $x \in X$, escolho $V_{x}$ tal que $x \in V_{x} \in \mathcal{V}_{n}$. Como $X$ é regular, posso escolher $B_{x} \in \mathcal{B}$ tal que $x \in B_{x} \subset \overline{B_{x}} \subset V_{x}$. Além disso, como $\mathcal{V}_{n}$ é localmente finita, existe 
$O_{x}$ vizinhança aberta de $x$ que intersecciona somente finitos elementos de $\mathcal{V}_{n}$. Definimos então a cobertura $\mathcal{O}_{n}=\left\{O_{x} \cap B_{x}: x \in X\right\}$. A partir de $\mathcal{O}_{n}$ usamos a paracompacidade de $X$ para criar $\mathcal{V}_{n+1}$ um refinamento aberto localmente finito. Temos então que $\mathcal{V}_{n+1}$ satisfaz as propriedades desejadas.

Terminada a construção das coberturas $\mathcal{V}_{n}$, definimos :

$$
\mathcal{V}=\left\{\bigcap_{n \in \omega} V_{n}: \forall n \in \omega, V_{n} \in \mathcal{V}_{n} \text { e } V_{n} \supset V_{n+1}\right\}
$$

Para cada elemento $\bigcap_{n \in \omega} V_{n} \in \mathcal{V}$, definimos $B_{n} \in \mathcal{B}$ para cada $n \in \omega$ como o elemento de $\mathcal{B}$ no item III. Temos que $\bigcap_{n \in \omega} V_{n}=\bigcap B_{n}$, e portanto $\mathcal{V}$ satisfaz a condição 1 do enunciado do lema. O item II garante que $\mathcal{V}$ satisfaz o item 2 .

Para mostrar que $\mathcal{V}$ cobre todo $X$, vamos usar um pouco de combinatória. Fixado $p \in X$, vamos construir a seguinte árvore: dado $n \in \omega$, um vértice da árvore no nível $n$ é um aberto $V_{n} \in \mathcal{V}_{n}$ tal que $p \in V_{n}$ e para qualquer nó $V_{n+1}$ no nível $n+1$ adjacente a $V_{n}$ existe um aberto $B_{n} \in \mathcal{B}$ tal que $V_{n+1} \subset B_{n} \subset \overline{B_{n}} \subset V_{n}$. Tal árvore é infinita pois, graça à condição III, dado qualquer $n \in \omega$ e um $V_{n} \in \mathcal{V}_{n}$ tal que $p \in V_{n}$, existe uma sequência finita $\left(V_{i}\right)_{i<n}$ tal que $\left(V_{i}\right)_{i \leq k}$ é um sub-ramo da árvore. Além disso, cada vértice da árvore possui finitas ramificações, pois as coberturas em questão são localmente finitas. Assim, pelo Lema de König (Teorema 1.1.10), temos que tal árvore possui um ramo infinito, e a intersecção dos abertos de tal ramo vai ser um elemento de $\mathcal{V}$ que contém $p$, como gostaríamos.

Agora temos as ferramentas necessárias para provar o principal teorema desta seção:

Teorema 3.2.2. Supondo a Hipótese do Continuo (CH), se para cada $i \in \omega$, $X_{i}$ é um espaço topológico compacto Hausdorff, então $\square_{i \in \omega} X_{i}$ é paracompacto se e somente se $L\left(\square_{i \in \omega} X_{i}\right) \leq \mathfrak{c}$.

Demonstração. Uma lado da equivalência na realidade não necessita da hipótese do contínuo. Suponha $\square_{i \in \omega} X_{i}$ paracompacto. Suponha $\square_{i \in \omega} X_{i}$ paracompacto. Dada $\mathcal{U}$ uma cobertura aberta de $\square_{i \in \omega} X_{i}$ e $\mathcal{B}$ a base usual de 
$\square_{i \in \omega} X_{i}$, aplicamos o lema 3.2 .1 para obter o refinamento aberto $\mathcal{U}^{\prime}$. Seja $Z=\prod_{i \in \omega} X_{i}$ com o produto de Tychonoff. Note que os elementos de $\mathcal{U}^{\prime}$ são fechados $G_{\delta}$ em $Z$, e portanto se encaixam nas hipóteses do teorema 1.2.22. Assim, temos que $\left|\mathcal{U}^{\prime}\right| \leq \mathfrak{c}$. Como todo refinamento caracteriza trivialmente uma subcobertura, temos que $L\left(\square_{i \in \omega} X_{i}\right) \leq \mathfrak{c}$, como queríamos

Para provar a recíproca precisaremos da hipótese do contínuo. Suponha $L\left(\square_{i \in \omega} X_{i}\right) \leq \mathfrak{c}$. Note que $L\left(\square_{i \in \omega} X_{i}\right)=L\left(\nabla_{i \in \omega} X_{i}\right)$, pois como a projeção $q: \square_{i \in \omega} X_{i} \longrightarrow \nabla_{i \in \omega} X_{i}$ é contínua e aberta, a cada cobertura aberta de $\square_{i \in \omega} X_{i}$ podemos associar uma cobertura aberta de $\nabla_{i \in \omega} X_{i}$ e vice-versa. Assumindo a hipótese do contínuo, temos $L\left(\nabla_{i \in \omega} X_{i}\right) \leq \aleph_{1}$. Pelo lema 3.1.4, temos que $\nabla_{i \in \omega} X_{i}$ é um $P_{\aleph_{1}}$-espaço. Concluímos então, pelo lema 1.2.28, que $\nabla_{i \in \omega} X_{i}$ é paracompacto e portanto $\square_{i \in \omega} X_{i}$ também o é, pelo Nabla Lemma (teorema 3.1.5).

A consequência de $\mathrm{CH}$ dada pelo teorema anterior é na realidade uma equivalência, como mostra o seguinte exemplo.

Exemplo 3.2.3. Existe um espaço compacto $X$ tal que $w(X)=\aleph_{2} e \square^{\omega} X$ não é normal.

A construção deste exemplo será independente da hipótese do contínuo. Para mostrar que esse exemplo garante que a Hipótese do Contínuo é equivalente ao teorema 3.2.2, construímos o espaço descrito no exemplo e supomos a negação da hipótese do contínuo (isto é, supomos válido $\aleph_{2} \leq \mathfrak{c}$ ).

Temos assim:

$$
L\left(\square^{\omega} X\right) \leq w\left(\square^{\omega}\right) \leq w(X)^{\aleph_{0}}=\aleph_{2}^{\aleph_{0}} \leq \mathfrak{c}^{\aleph_{0}}=\left(2^{\aleph_{0}}\right)^{\aleph_{0}}=2^{\aleph_{0} \times \aleph_{0}}=2^{\aleph_{0}}=\mathfrak{c}
$$

Assim, temos que $X$ e $\square^{\omega} X$ se encaixam nas hipóteses do teorema 3.2.2. Todavia, $\square^{\omega} X$ é Haussdorf, mas não é normal. Portanto $\square^{\omega} X$ não é paracompacto, testemunhando a necessidade da Hipótese do Contínuo para o resultado do teorema.

Para construir esse exemplo, precisaremos de mais alguns conceitos:

Definição 3.2.4. Dado um espaço topológico $X$, denotamos por $X_{\delta}$ o espaço topológico formado pelo mesmo conjunto de pontos de $X$ com a topologia 
gerada por todos os conjuntos $G_{\delta}$ de $X$. Chamamos $X_{\delta}$ de $G_{\delta}$-modificação de $X$, ou topologia $G_{\delta}$ de $X$.

Lema 3.2.5. Dado um espaço topológico Hausdorff $X$, temos que $X_{\delta}$ é homeomorfo a um subespaço fechado de $\square^{\omega} X$. Da mesma forma, $X_{\delta}$ também é homeomorfo a um subespaço fechado de $\nabla^{\omega} X$

Demonstração. Defino a função $\varphi: X_{\delta} \longrightarrow \nabla^{\omega} X$ dada por $\varphi(x)=q(\widehat{x})\left(_{-}\right.$ como na definição 2.2.6). É fácil ver que $\varphi$ é injetora, pois dados $x, y \in X_{\delta}$ distintos, $\widehat{x}$ e $\widehat{y}$ são diferentes em todas as coordenadas.

Consideremos a restrição de $\varphi$ ao seu domínio (ou seja, $\left.\varphi: X_{\delta} \longrightarrow \varphi\left(X_{\delta}\right) \subset \nabla^{\omega} X\right)$. Assim, temos $\varphi$ bijetora.

Vamos mostrar que $\varphi$ é contínua. Note que $\varphi$ nada mais é do que a composição da função $\widehat{\cdot}: X \rightarrow \square^{\omega} X$ com a projeção $q: \square^{\omega} X \rightarrow \nabla^{\omega} X$. Como por definição temos $q$ contínua, então basta provar que ${ }_{-}: X \rightarrow \square^{\omega} X$ é contínua. Seja $A=q\left(\prod_{i \in \omega} A_{i}\right)$, onde para todo $i \in \omega$ temos $A_{i} \subset X$ aberto. Note que a imagem inversa de $A$ por $x \mapsto \widehat{x}$ é $\bigcap_{i \in \omega} A_{i}$, o qual é um conjunto $G_{\delta}$ em $X$ e, portanto, é aberto em $X_{\delta}$.

Agora vamos mostrar que $\varphi$ é uma função aberta. Seja $V$ um aberto em $X_{\delta}$. Podemos supor $V$ um aberto básico escrever $V=\bigcap_{n \in \omega} V_{n}$, com $V_{n}$ aberto em $X$ para cada $n \in \omega$. Definimos então $\widehat{V_{n}}=\left(V_{n}\right)^{\omega}$ para cada $n \in \omega$. Note que $\widehat{V_{n}}$ é aberto em $\square^{\omega} X, \operatorname{logo} q\left(\widehat{V_{n}}\right)$ é aberto em $\nabla^{\omega} X$. Como $\nabla^{\omega} X$ é um P-espaço, temos que $\bigcap_{n \in \omega} q\left(\widehat{V_{n}}\right)$ é aberto em $\nabla^{\omega} X$. Note que $\varphi(V)=\left(\bigcap_{n \in \omega} q\left(\widehat{V_{n}}\right)\right) \cap \varphi\left(X_{\delta}\right), \operatorname{logo} \varphi$ é de fato um homeomorfismo.

Note também que $\varphi\left(X_{\delta}\right)$ é fechado em $\nabla^{\omega} X$. Seja $\widehat{X}=\{\widehat{x}: x \in X\}$ e fixe $p \in\left(\square^{\omega} X\right) \backslash \widehat{X}$. Existem $n, m \in \omega$ tais que $p_{n} \neq p_{m}$. Como $X$ é Hausdorff, existem abertos disjuntos $U, V$ tais que $p_{n} \in U$ e $p_{m} \in V$. Considere então a caixa aberta $W=\prod_{i \in \omega} W_{i}$ definida por

$$
W_{i}= \begin{cases}U & \text { se } i=n \\ V & \text { se } i=m \\ X & \text { caso contrário }\end{cases}
$$

Note que $W$ é uma vizinhança aberta de $p$ que comprova que $\widehat{X}$ é fechado 
em $\square^{\omega} X$. Assim, temos que $\varphi(X)=q(\widehat{X})$ é fechado, pois $q$ é uma função fechada.

Por fim, para mostrar o mesmo resultado relativo a $\square^{\omega} X$, vamos construir um homeomorfismo $\psi: \nabla^{\omega} X \rightarrow \psi(X) \subset \square^{\omega} X$. Para cada $Z \in \nabla^{\omega} X$, $\psi$ escolhe um representante da classe $Z$, com a restrição de que se existe $\widehat{x} \in \widehat{X}$ tal que $\widehat{x} \in Z$, então definimos $\psi(Z)=\widehat{x}$. A função $\psi$ com tal restrição está bem definida pois como vimos na construção de $\varphi$, existe no máximo um $\widehat{x} \in \widehat{X}$ tal que $\widehat{x} \in Z$. Como as classes são disjuntas, temos que $\psi$ é injetora, logo bijetora. Note também que a inversa de $\psi$ nada mais é do que $q$. Como $q$ é contínua e aberta, temos que $\psi$ é um homeomorfismo. Definimos então o homeomorfismo entre $X_{\delta}$ e um subespaço de $\square^{\omega} X$ como a composição $\psi \circ \varphi$. Note que $\psi \circ \varphi(X)=\widehat{X}$, o qual já vimos é fechado em ${ }^{\omega} X$, completando a demonstração.

Demonstração do exemplo 3.2.3. Definimos $X=\prod^{\omega_{2}}[0,1]$ equipado com a topologia produto usual (produto de Tychonoff). Temos que $X$ é compacto, e como $[0,1]$ possui base enumerável, temos que $w(X)=\aleph_{2}$. Pelo lema 3.2.5, temos que $X_{\delta}$ é homeomorfo a um subespaço fechado de $\square^{\omega} X$. Como a normalidade é preservada por subespaços fechados, basta provar que $X_{\delta}$ não é normal.

Como $[0,1]_{\delta}$ é discreto, segue que $X_{\delta}$ e $\left(\prod^{\omega_{2}}[0,1]_{\delta}\right)_{\delta}$ tem a mesma topologia.

Considere $Z$ subespaço de $[0,1]_{\delta}$ tal que $|Z|=\aleph_{1}$. Definimos $Y=\prod^{\omega_{2}} Z$. Como $Y_{\delta}$ é subespaço fechado de $X_{\delta}$, reduzimos novamente o problema a provar que $Y_{\delta}$ não é normal. Para tanto, vamos construir dois conjuntos fechados que não satisfazem a condição de normalidade:

Primeiramente, indexamos $Z=\left\{z_{\alpha}: \alpha \in \omega_{1}\right\}$. Note que todo $y \in Y$ é uma função $y: \omega_{2} \longrightarrow Z$, e $Z$ é equipotente a $\omega_{1}$. Pelo princípio da casa dos pombos, existe um ordinal $\alpha \in \omega_{1}$ tal que $\left|y^{-1}\left(z_{\alpha}\right)\right|>1$. Assim, podemos construir o conjunto $C(0)$ dos $y \in Y$ tais que $\left|y^{-1}\left(z_{\alpha}\right)\right|>1$ somente para 
$\alpha=0$, e da mesma forma construímos $C(1)$. Em outras palavras,

$$
\begin{aligned}
& C(0)=\left\{y \in Y: \forall \alpha \in \omega_{1},\left(\left|y^{-1}\left(z_{\alpha}\right)\right|>1 \Rightarrow \alpha=0\right)\right\} \\
& C(1)=\left\{y \in Y: \forall \alpha \in \omega_{1},\left(\left|y^{-1}\left(z_{\alpha}\right)\right|>1 \Rightarrow \alpha=1\right)\right\}
\end{aligned}
$$

Note que as funções constantes $z_{0}$ e $z_{1}$ pertencem a $C(0)$ e $C(1)$ respectivamente, $\log 0 C(0), C(1) \neq \emptyset$. Também é fácil ver que $C(0) \cap C(1)=\emptyset$, pois se existisse $p \in C(0) \cap C(1)$, teríamos que $p$ é uma função injetora. Temos ainda que esses conjuntos são fechados: dado $y \in Y \backslash C(0)$, temos $\beta_{1}, \beta_{2} \in \omega_{2}$ distintos tais que $\beta_{1}, \beta_{2} \neq 0$ e $y\left(\beta_{1}\right)=y\left(\beta_{2}\right)$. Considere o aberto $V=\prod_{\lambda \in \omega_{2}} V_{\lambda}$ dado por:

$$
V_{\lambda}=\left\{\begin{array}{cl}
\left\{y\left(\beta_{1}\right)\right\} & \text { se } \lambda=\beta_{1} \\
\left\{y\left(\beta_{1}\right)\right\} & \text { se } \lambda=\beta_{2} \\
Z & \text { caso contrário }
\end{array} .\right.
$$

O conjunto $V$ testemunha que $C(0)$ é fechado, e da mesma forma temos $C(1)$ também o é.

Suponhamos que exista um aberto $G$ tal que $C(0) \subset G \subset \bar{G} \subset Y_{\delta} \backslash C(1)$. Para chegar ao absurdo, utilizaremos uma base $\mathcal{U}$ de $Y_{\delta}$ tal que para todo $U=\prod_{\lambda \in \omega_{2}} \in \mathcal{U}$ e todo $\lambda \in \omega_{2}$ temos

- $U_{\lambda} \neq Z \Rightarrow\left|U_{\lambda}\right|=1$;

- $|\operatorname{spt}(U)|=\aleph_{0}$ e $\omega_{1} \cap \operatorname{spt}(U)$ é um ordinal infinito.

De fato, para satisfazer a primeira condição, usamos que $[0,1]_{\delta}$ é discreto. Para a segunda, usamos que $Y_{\delta}$ é P-espaço. Com $\mathcal{U}$ em mãos, para cada $\alpha \in \omega_{1}$, vamos construir recursivamente um aberto $U_{\alpha} \in \mathcal{U}$, um ordinal $\eta_{\alpha}<\omega_{1}$, uma função bijetora $\varphi_{\alpha}: \eta_{\alpha} \longrightarrow \operatorname{spt}\left(U_{\alpha}\right)$ e um ponto $u_{\alpha} \in C(0)$ que satisfaçam as condições:

1. $\omega_{1} \cap \operatorname{spt}\left(U_{\alpha}\right)=\eta_{\alpha}$

2. $\alpha<\eta_{\alpha}<\omega_{1}$ 
3. $\beta<\alpha \Rightarrow \eta_{\beta}<\eta_{\alpha}$

4. $\beta<\alpha \Rightarrow \varphi_{\beta}=\varphi_{\alpha} \uparrow \beta$

5. $u_{\alpha} \in U_{\alpha} \subset G$

6. $\forall \lambda \in \omega_{2}, u_{\alpha}(\lambda)= \begin{cases}z_{\gamma} & \text { se } \exists \beta<\alpha, \exists \gamma \in \eta_{\alpha}, \varphi_{\beta}(\gamma)=\lambda \\ z_{0} & \text { caso contrário }\end{cases}$

Começamos construindo o caso $\alpha=0$. Definimos $u_{0}$ como a constante $z_{0}$. Escolhemos $U_{0} \in \mathcal{U}$ tal que $u_{0} \in U_{0}$. Fixamos $\eta_{0}=\omega_{1} \cap \operatorname{spt}\left(U_{0}\right)$ como no item 1. A segunda condição satisfeita pela base $\mathcal{U}$ garante que $\eta_{0}$ seja um ordinal e que exista uma bijeção $\varphi_{0}: \eta_{0} \longrightarrow \operatorname{spt}\left(U_{0}\right)$, já que ambos os conjuntos possuem cardinalidade $\aleph_{0}$.

Agora seja $\nu \in \omega_{1}$ tal que para todo $\alpha<\nu$ já estejam construídos $U_{\alpha}, \eta_{\alpha}, \varphi_{\alpha}$ e $u_{\alpha}$. Definimos então $S_{\nu}=\bigcup_{\alpha<\nu} \operatorname{spt}\left(U_{\alpha}\right), \theta_{\nu}=\bigcup_{\alpha<\nu} \eta_{\alpha}$ e $\psi_{\nu}=$ $\bigcup_{\alpha<\nu} \varphi_{\alpha}$. O item 4 garante que essa colagem é válida e que $\psi_{\nu}: \theta \longrightarrow S_{\nu}$ é uma função bijetora. Definimos também:

$$
u_{\nu}(\lambda)= \begin{cases}z_{\gamma} & \text { se } \exists \gamma<\theta_{\nu}, \psi_{\nu}(\gamma)=\lambda \\ z_{0} & \text { caso contrário }\end{cases}
$$

Note que como $\psi_{\nu}$ é bijetora, temos que $u_{\nu} \in C(0)$. Temos então que $u_{\nu}$ foi escolhido distinto dos $u_{\alpha}$ anteriores, ou seja, $u_{\nu} \notin\left\{u_{\alpha}: \alpha<\nu\right\}$. Usando o fato de $\mathcal{U}$ ser base de $Y_{\delta}$, podemos escolher $U_{\nu} \in \mathcal{U}$ tal que $u_{\nu} \in U_{\nu}$ e $S_{\nu} \cup\left(\theta_{\nu}+\omega\right) \subset \operatorname{spt}\left(U_{\nu}\right)$. Tal inclusão é possível pois $S_{\nu} \cup\left(\theta_{\nu}+\omega\right)$ é enumerável, já que tanto $S_{\nu}$ quanto $\theta_{\nu}$ são uniões enumeráveis de conjuntos enumeráveis. Assim, definimos $\eta_{\nu}=\omega_{1} \cap \operatorname{spt}\left(U_{\nu}\right)$. Como $\operatorname{spt}\left(U_{\nu}\right) \backslash S_{\nu}$ é enumerável, podemos extender $\psi_{\nu}$ a uma função $\varphi_{\nu}: \eta_{\nu} \longrightarrow \operatorname{spt}\left(U_{\nu}\right)$. Temos então as sequências construídas satisfazendo os itens de 1 a 6 .

Com as sequências prontas, definimos $S=\bigcup_{\alpha \in \omega_{1}} \operatorname{spt}\left(U_{\alpha}\right)$ e $\Phi=\bigcup_{\alpha \in \omega_{1}} \varphi_{\alpha}$. Os itens 2, 3 e 4 garantem que $\Phi$ é de fato uma função bijetora. Assim, podemos construir um ponto $x \in Y_{\delta}$ dado por:

$$
x(\lambda)= \begin{cases}z_{\gamma} & \text { se } \lambda \in S \text { e } \Phi(\gamma)=\lambda \\ z_{1} & \text { caso contrário }\end{cases}
$$


O fato de $\Phi$ ser bijetora garante que $x \in C(1)$. Pela nossa suposição, temos $C(1) \cap \bar{G}=\emptyset$, então existe $V \in \mathcal{U}$ tal que $x \in V \subset Y_{\delta} \backslash \bar{G}$. Pela construção de $\mathcal{U}$, temos que $S \cap \operatorname{spt}(V)$ é um ordinal infinito enumerável, $\operatorname{logo}$ existe o menor ordinal $\alpha$ tal que $S \cap \operatorname{spt}(V) \subset \omega_{1} \cap \operatorname{spt}\left(U_{\alpha}\right)$. Pelos itens 1 e 2 , temos que $\omega_{1} \subset S$, e portanto $S \cap \operatorname{spt}(V) \subset \operatorname{spt}\left(U_{\alpha}\right)$.

Para chegar à contradição, vamos mostrar que $U_{\alpha} \cap V \neq \emptyset$. Denotemos $V=\prod_{\lambda \in \omega_{2}} V_{\lambda}$. Note que

$$
U_{\alpha} \cap V=\prod_{\lambda \in \omega_{2}}\left(V_{\lambda} \cap\left(U_{\alpha}\right)_{\lambda}\right)
$$

Portanto, basta provar que para cada $\lambda \in \omega_{2}$ temos $V_{\lambda} \cap\left(U_{\alpha}\right)_{\lambda} \neq \emptyset$. Se $\lambda \notin \operatorname{spt}\left(U_{\alpha}\right)$, temos $\left(U_{\alpha}\right)_{\lambda}=Z$, portanto $\emptyset \neq V_{\lambda} \subset\left(U_{\alpha}\right)_{\lambda}$. Da mesma forma, se $\lambda \notin \operatorname{spt}(V)$, temos $V_{\lambda}=Z$, portanto $\emptyset \neq\left(U_{\alpha}\right)_{\lambda} \subset V_{\lambda}$. Caso contrário, temos $\lambda \in \operatorname{spt}\left(U_{\alpha}\right) \cap \operatorname{spt}(V)$. Neste caso, temos

$$
V_{\lambda}=\left(U_{\alpha}\right)_{\lambda}=\{x(\lambda)\}=\left\{u_{\alpha}(\lambda)\right\}
$$

Assim, em cada um dos casos, temos $V_{\lambda} \cap\left(U_{\alpha}\right)_{\lambda} \neq \emptyset$, como gostaríamos.

\subsection{Pequenos cardinais}

Vimos uma condição para a paracompacidade de produtos caixa enumeráveis de espaços compactos sob a Hipótese do Contínuo. Agora veremos algumas condições sob hipóteses mais fracas.

Podemos então trabalhar com cardais intermediários entre $\aleph_{0}$ e $\mathfrak{c}$, os quais se tornam trivialmente iguais a $\mathfrak{c}$ sob a Hipótese do Contínuo. É comum referir a tais cardinais por pequenos cardinais. Neste texto, trabalharemos com dois desses cardinais.

Definição 3.3.1. Sejam $f, g: \omega \longrightarrow \omega$. Dizemos que:

- $f \leq^{*} g$ se $\{n \in \omega: f(n)>g(n)\}$ é finito;

- $f<^{*} g$ se $f \leq^{*} g$ e não for verdade que $g \leq^{*} f$. 
Definição 3.3.2. Seja $W \subset \omega^{\omega}$ uma família de funções de $\omega$ em $\omega$. Dizemos que $W$ é:

- ilimitada se $\forall f \in \omega^{\omega}, \exists g \in W, g \mathbb{Z}^{*} f$ (caso contrário, $W$ é dita limitada);

- dominante (sobre $\left.\omega^{\omega}\right)$ se $\forall f \in \omega^{\omega}, \exists g \in W, f \leq^{*} g$.

Com esses conceitos definimos dois cardinais:

- $\mathfrak{b}=\min \{|W|: W$ é ilimitado $\} e$

- $\mathfrak{d}=\min \{|W|: W$ é dominante $\}$

Outro conceito importante para a aritmética cardinal que usaremos é o das escalas.

Definição 3.3.3. Uma $\lambda$-escala é uma sequência $\left\{f_{\alpha} \in \omega^{\omega}: \alpha<\lambda\right\}$ dominante e estritamente crescente considerando a ordem $\leq *$.

Apresentamos então algumas propriedades dos cardinais $\mathfrak{b}$ e $\mathfrak{d}$ :

\section{Proposição 3.3.4.}

1. $\aleph_{1} \leq \mathfrak{b} \leq \mathfrak{d} \leq \mathfrak{c}$

2. $\mathfrak{b}$ é um cardinal regular.

3. $\mathfrak{b}=\mathfrak{d}$ se e somente se existe uma $\mathfrak{b}$-escala.

Demonstração. 1. Como d representa uma cardinalidade de um subconjunto de $\omega^{\omega}$, temos que $\mathfrak{d} \leq\left|\omega^{\omega}\right|=2^{\aleph_{0}}=\mathfrak{c}$. Além disso, pela definição temos que toda família dominante é ilimitada, logo $\mathfrak{b} \leq \mathfrak{d}$. Por fim, vamos mostrar que nenhuma família enumerável é ilimitada, e portanto $\aleph_{1} \leq \mathfrak{b}$. Para isso, vamos usar o argumento da diagonal. Seja $\left\{r_{n}: n \in \omega\right\} \subset \omega^{\omega}$. Definimos então $s: \omega \longrightarrow \omega$ dada por $s(i)=\sum_{n<i} r_{n}(i)$. Assim, temos $\forall n \in \omega, r_{n} \leq^{*} s$, e portanto $\left\{r_{n}: n \in \omega\right\}$ é uma família limitada, como queríamos. 
2. Seja $\left\{r_{\alpha} \in \omega^{\omega}: \alpha<\mathfrak{b}\right\}$ uma família ilimitada. Vamos construir recursivamente uma família $\left\{s_{\alpha} \in \omega^{\omega}: \alpha<\mathfrak{b}\right\}$ ilimitada bem ordenada pela ordem $\leq^{*}$ e de cumprimento $\mathfrak{b}$. Começamos com $s_{0}=r_{0}$. Dado $\alpha<\mathfrak{b}$, supomos $r_{\beta}$ construído para todo $\beta<\alpha$. Como a família $\left(r_{\beta}+s_{\beta}\right)_{\beta<\alpha}$ tem comprimento $\alpha<\mathfrak{b}$, tal família é limitada, portanto podemos escolher $s_{\alpha}$ como a função que limita tal família. Concluída a construção, temos que o fato de $\left(r_{\alpha}\right)_{\alpha<\mathfrak{b}}$ ser ilimitada garante que $\left(s_{\alpha}\right)_{\alpha<\mathfrak{b}}$ também o seja. Qualquer subconjunto cofinal de $S=\left\{s_{\alpha}: \alpha<\mathfrak{b}\right\}$ também será ilimitado, portanto precisa ter cardinalidade $\mathfrak{b}, \log \operatorname{cf}(\mathfrak{b})=\operatorname{cf}(S)=\mathfrak{b}$

3. Se existe uma $\mathfrak{b}$-escala, esta é uma família dominante de tamanho $\mathfrak{b}$, $\operatorname{logo}$ pelo item 1 temos $\mathfrak{b}=\mathfrak{d}$. Por outro lado, se $\mathfrak{b}=\mathfrak{d}$, então existe uma família dominante de tamanho $\mathfrak{b}$, a partir da qual podemos construir uma $\mathfrak{b}$ escala usando a recursão da demonstração do item 2.

Não trabalharemos com o Axioma de Martin (MA) neste texto, porém é um resultado conhecido que MA implica $\mathfrak{b}=\mathfrak{c}$ (para este e outros resultados sobre MA, consultar [Jec03]). Note que a proposição 3.3.4 possui a seguinte consequência direta: $\mathfrak{b}=\mathfrak{c}$ implica que $\mathfrak{c}$ é regular e que existe uma c-escala. Por sua vez, a hipótese de que existe uma c-escala e c é regular implica que $\mathfrak{d}=\mathfrak{c}$. No entanto a reciproca não é verdadeira, uma vez que $\mathfrak{d}=\mathfrak{c}$ não implica necessariamente a existência de alguma $\lambda$-escala, pois $\mathfrak{d}=\mathfrak{c}$ junto com a inexistência de uma c-escala é consistente com ZFC [Hec74].

Teorema 3.3.5. Se $X_{i}$ é primeiro-enumerável para todo $i \in \omega$, então $\nabla_{i \in \omega} X_{i}$ é um $P_{\mathfrak{b}}$-espaço.

Demonstração. Seja $q(p) \in \nabla_{i \in \omega} X_{i}$. Sejam $\kappa<\mathfrak{b}$ um ordinal e $U^{\alpha}=$ $q\left(\prod_{i \in \omega} U_{i}^{\alpha}\right)$ uma vizinhança aberta básica de $q(p)$ para cada $\alpha<k$. Vamos mostrar que $\bigcap_{\alpha<\kappa} U^{\alpha}$ é vizinhança de $q(p)$.

Fixemos para cada $n \in \omega$ uma base local $\left\{V_{i}^{j}: j \in \omega\right\}$ de $p_{i} \in X_{i}$ tal que $\forall j \in \omega, V_{i}^{j+1} \subset V_{i}^{j}$. Para cada $\alpha<\kappa$, podemos construir uma função $f_{\alpha}: \omega \longrightarrow \omega$ tal que para cada $i \in \omega$ temos $V_{i}^{f_{\alpha}(i)} \subset U_{i}^{\alpha}$. Assim, temos para cada $\alpha<\kappa$ que $q\left(\prod_{i \in \omega} V_{i}^{f_{\alpha}(i)}\right) \subset U_{\alpha}$. Com isso, definimos 
$W=\left\{f_{\alpha}: \alpha<\kappa\right\} \subset \omega^{\omega}$. Como $|W|=\kappa<\mathfrak{b}$, temos que a família $W$ de funções não é dominante. Então existe uma função $g: \omega \longrightarrow \omega$ tal que $\forall f \in W, f \leq^{*} g$. Assim, temos que $q(p) \in q\left(\prod_{i \in \omega} V_{i}^{g(i)}\right) \subset \bigcap_{\alpha<\kappa} U^{\alpha}$, pois para cada $\alpha<\kappa$, vale

$$
\left\{i: V_{i}^{g(i)} \not \subset U_{i}^{\alpha}\right\} \subset\left\{i: f_{\alpha}(i)<g(i)\right\}
$$

o qual é finito, o que conclui a demonstração.

Corolário 3.3.6. Se $\mathfrak{b}=\mathfrak{c}, X_{i}$ é compacto, Hausdorff e primeiro enumerável para todo $i \in \omega$, então $\square_{i \in \omega} X_{i}$ é paracompacto.

Demonstração. Como cada $X_{i}$ é compacto Hausdorff e primeiro enumerável, pelo Teorema de Arhangelskii (Teorema 1.2.21), temos que $w\left(X_{i}\right) \leq\left|X_{i}\right| \leq$ $2^{\aleph_{0}}=\mathfrak{c}$. Note que

$$
L\left(\nabla_{i \in \omega} X_{i}\right) \leq w\left(\nabla_{i \in \omega} X_{i}\right)=w\left(\square_{i \in \omega} X_{i}\right) \leq \prod_{i \in \omega} w\left(X_{i}\right) \leq \mathfrak{c}^{\omega}=\mathfrak{c}
$$

Aplicando o teorema 3.3.5 junto com o lema 1.2.28, obtemos que $\nabla_{i \in \omega} X_{i}$ é paracompacto. Concluímos aplicando o nabla lema (teorema 3.1.5).

Podemos reduzir a hipótese do resultado anterior para $\mathfrak{d}=\mathfrak{c}$. A demonstração, por Judith Roitman [Roi79] utiliza a seguinte propriedade do cardinal $\mathfrak{d}$ (a versão que usaremos está em [Wil84], mas é equivalente à de Roitman):

Lema 3.3.7. Sejam $W \subset \omega^{\omega}$ e $\mathcal{A} \subset[\omega]^{\omega}$ tais que $|\mathcal{A}|+|W|<\mathfrak{d}$. Então para cada $f \in W$ e cada $A \in \mathcal{A}$, existe uma função $g: \omega \longrightarrow \omega$ tal que o conjunto $\{n \in A: f(n)<g(n)\}$ é infinito.

Demonstração. Para cada par $(f, A) \in W \times \mathcal{A}$, definimos a função $f_{A} \in \omega^{\omega}$ dada por $f_{A}(n)=\sum_{i=0}^{k_{n}} f(i)$ para cada $n \in \omega$, onde $k_{n}=\min \{j \in A: n<j\}$.

Definindo $W_{\mathcal{A}}=\left\{f_{A}: f \in W, A \in \mathcal{A}\right\}$, como $\left|W_{\mathcal{A}}\right|<\mathfrak{d}$, podemos encontrar uma $g \in \omega^{\omega}$ crescente tal que $g \mathbb{Z}^{*} f_{A}$ para todo $f_{A} \in W_{\mathfrak{A}}$. Como para cada $n \in \omega$ temos que $n \leq i \leq k_{n}$ e $f_{A}(n)<g(n)$ implicam que $f(i)<g(n)<g(i)$, concluímos que $g$ é de fato a função que procuramos. 
Teorema 3.3.8. Se $\mathfrak{d}=\mathfrak{c}, X_{i}$ é Lindelöff, regular e primeiro-enumerável para todo $i \in \omega$, então $\nabla_{i \in \omega} X_{i}$ é ultraparacompacto.

Demonstração. Inicialmente, da mesma maneira que no corolário 3.3.6, te$\operatorname{mos}$ que $\left|X_{i}\right| \leq \mathfrak{c}$ para cada $i \in \omega$, do que concluímos que $\left|\nabla_{i \in \omega} X_{i}\right| \leq \mathfrak{c}$. Portanto podemos escrever $\nabla_{i \in \omega} X_{i}=\left\{x_{\alpha}: \alpha<\mathfrak{c}\right\}$. Além disso, como cada $X_{i}$ é primeiro enumerável, para cada $x_{i} \in X_{i}$ temos uma base local decrescente $\left\{v_{x_{i}, n}: n \in \omega\right\}$. Dada um ponto $x=\left(x_{i}\right)_{i \in \omega} \in \square_{i \in \omega} X_{i}$ e uma função $f: \omega \longrightarrow \omega$ qualquer, denotaremos $u_{x, f}=\nabla_{i \in \omega} v_{x_{i}, f(i)}$. Note que esta notação tem a seguinte propriedade: dados $x, y \in \nabla_{i \in \omega} X_{i}$ e $f, g: \omega \longrightarrow \omega$, temos:

$$
\left|\left\{i \in \omega: v_{x_{i}, f(i)} \cap v_{y_{i}, g(i)}=\emptyset\right\}\right| \geq \aleph_{0} \Longleftrightarrow u_{x, f} \cap u_{y, g}=\emptyset
$$

Seja $\mathfrak{U}$ uma cobertura aberta de $\nabla_{i \in \omega} X_{i}$. Vamos construir um refinamento $\mathfrak{U}^{\prime}=\left\{U_{\alpha}: \alpha<\mathfrak{c}\right\}$ de $\mathfrak{U}$ dois-a-dois disjunto (a menos de repetição). Como $\nabla_{i \in \omega} X_{i}$ é regular e P-espaço, escolhendo $V \in \mathfrak{U}$ tal que $x_{0} \in V$, podemos construir uma sequência estritamente crescente (pela ordem termo a termo, como definida na observação $4.1 .4\left\{f_{0, n} \in \omega^{\omega}: n \in \omega\right\}$ tal que $\bigcap_{n \in \omega} u_{x_{0}, f_{0, n}}$ seja um aberto fechado contido em $V$. Definimos $U_{0}=\bigcap_{n \in \omega} u_{x_{0}, f_{0, n}}$.

Para algum $\alpha<\mathfrak{c}$, suponhamos $U_{\beta}$ já construído para todo $\beta<\alpha$. Se $x_{\alpha} \in \bigcup_{\beta<\alpha} U_{\beta}$, definimos $U_{\alpha}=U_{\beta}$, para algum $\beta<\alpha$ tal que $x_{\alpha} \in U_{\beta}$. Suponhamos agora o contrário. Para cada $\beta<\alpha$, como $U_{\beta}$ é fechado e $\nabla_{i \in \omega} X_{i}$ é regular, podemos encontrar uma função $g_{\beta}: \omega \longrightarrow \omega$ tal que $U_{\beta} \cap u_{x_{\alpha}, g_{\beta}}=\emptyset$. Como a sequência $\left(f_{\beta, n}\right)_{n \in \omega}$ é crescente para $\beta<\alpha$, podemos encontrar um natural $n_{\beta}$ tal que $u_{x_{\alpha}, g_{\beta}} \cap u_{x_{\beta}, f_{\beta, n_{\beta}}}=\emptyset$. Pela propriedade que citamos da notação utilizada, temos que o conjunto

$$
A_{\beta}=\left\{i \in \omega: v_{\left(x_{\alpha}\right)_{i}, g_{\beta}(i)} \cap v_{\left(x_{\beta}\right)_{i}, f_{\beta, n_{\beta}}(i)}=\emptyset\right\}
$$

seja infinito. Pelo lema 3.3.7 junto com a hipótese que $\mathfrak{d}=\mathfrak{c}$, podemos encontrar uma função $h: \omega \longrightarrow \omega$ tal que para cada $\beta<\alpha$ o conjunto $\left\{j \in A_{\beta}: g_{\beta}(j)<h(j)\right\}$ é infinito. Pela notação utilizada, temos que $u_{x_{\alpha}, h} \cap$ $U_{\beta}=\emptyset$ para cada $\beta<\alpha$. Escolhemos então $V \in \mathfrak{U}$ tal que $x_{\alpha} \in V$ e construímos, da mesma maneira que anteriormente, uma sequência crescente 
de funções $\left\{h_{n} \in \omega^{\omega}: n \in \omega\right\}$ tal que $h_{0}=h$ e $U=\bigcap_{n \in \omega} u_{x_{\alpha}, h_{n}} \subset V$ seja um aberto-fechado. Definimos então $U_{\alpha}=U$ e assim concluímos a construção por indução.

Corolário 3.3.9. Se $\mathfrak{d}=\mathfrak{c}, X_{i}$ é compacto, regular e primeiro-enumerável para todo $i \in \omega$, então $\square_{i \in \omega} X_{i}$ é paracompacto.

No caso intermediário $\mathfrak{b}=\mathfrak{d}$, o seguinte teorema de van Douwen [vD80] nos mostra que uma hipótese suficiente para obter a paracompacidade de produtos caixa enumerável é a metrizabilidade e compacidade dos fatores.

Teorema 3.3.10. O produto caixa de uma quantidade enumerável de espaços metrizáveis compactos é paracompacto se $\mathfrak{b}=\mathfrak{d}$.

Este teorema é uma simples consequência do seguinte lema, demonstrado, por exemplo, por Roitman em [Roi11]:

Lema 3.3.11. Suponha $\mathfrak{b}=\mathfrak{d}$. Então o produto nabla enumerável de espaços metrizáveis compactos é $\mathfrak{b}$-metrizável.

Demonstração. Pelo item 3 da proposição 3.3.4, existe uma família $\left\{f_{\alpha} \in \omega^{\omega}: \alpha<\mathfrak{b}\right\}$ a qual é uma $\mathfrak{b}$-escala. Para cada $q(x) \in \nabla_{i \in \omega} X_{i}$ e cada $\alpha<\mathfrak{b}$, definimos

$$
u_{q(x), \alpha}=\bigcap_{n \in \omega} \nabla_{i \in \omega} B_{i}\left(x_{i}, \frac{1}{2^{\left(n+f_{\alpha}(i)\right)}}\right)
$$

onde $B_{i}\left(x_{i}, r\right)$ é a bola de centro $x_{i}$ e raio $r$ em $X_{i}$. Temos então que $\mathcal{B}_{q(x)}=$ $\left\{u_{q(x), \alpha}: \alpha<\mathfrak{b}\right\}$ é a base local de $q(x)$ a qual testemunha que $\nabla_{i \in \omega} X_{i}$ é um espaço $\mathfrak{b}$-metrizável.

Continuando com os teoremas sobre a paracompacidade de produtos caixa utilizando hipóteses sobre pequenos cardinais, apresentamos um teorema de Scott Williams [Wil84], originalmente demonstrado através de uniformidades. No entanto, seguiremos a demonstração de Judith Roitman em [Roi11], a qual utiliza submodelos elementares. Ambas as demonstrações se baseiam em construir uma base para $\nabla_{i \in \omega} X_{i}$ que testemunhe a $\omega_{1}$-metrizabilidade de tal espaço. 
Teorema 3.3.12. Suponha $\mathfrak{d}=\aleph_{1}$. Se $X_{i}$ é um espaço compacto Hausdorff com $w\left(X_{i}\right) \leq \aleph_{1}$ para cada $i \in \omega$, então $\square_{i \in \omega} X_{i}$ é paracompacto.

Demonstração. Vamos mostrar que $\nabla_{i \in \omega} X_{i}$ é $\omega_{1}$-metrizável, de maneira que os teoremas 3.1.5 e 1.2.31 completam a demonstração.

Primeiramente, usamos a hipótese $w\left(X_{i}\right) \leq \aleph_{1}$ para selecionar uma base $\mathcal{C}_{i}$ enumerável para cada $i \in \omega$. Vamos construir, para cada $i \in \omega$, uma sequência crescente $\left(\mathcal{C}_{i, \alpha}\right)_{\alpha<\omega_{1}}$ de subconjuntos enumeráveis de $\mathcal{C}_{i}$. Construiremos tal sequência de maneira que para cada $\alpha<\omega_{1}, \mathcal{C}_{i, \alpha}$ seja base de um subespaço $X_{i, \alpha} \subset X_{i}$, de maneira que $X_{i}=\bigcup_{\alpha<\omega_{1}} X_{i, \alpha}$.

Para construir $\left(\mathcal{C}_{i, \alpha}\right)_{\alpha<\omega_{1}}$, vamos usar o conceito de submodelos elementares. Primeiramente, consideramos $H\left(\omega_{2}\right)$ a coleção de todos os conjuntos cujo fecho transitivo possui cardinalidade menor que $\omega_{2}$. Pelo Teorema de Löwenheim-Skolem, podemos construir $\left(M_{\alpha}\right)_{\alpha<\omega_{1}}$ uma sequência contínua crescente de submodelos elementares enumeráveis de $H\left(\omega_{2}\right)$, de maneira que $\forall i \in \omega, \mathcal{C}_{i} \in \bigcup_{\alpha<\omega_{1}} M_{\alpha}$. Definimos então $\mathcal{C}_{i, \alpha}=\mathcal{C}_{i} \cap M_{\alpha}$.

Para cada $\mathcal{C}_{i, \alpha}$, definimos $X_{i, \alpha}=\bigcup \mathcal{C}_{i, \alpha}$. Como $\left(\mathcal{C}_{i, \alpha}\right)_{\alpha<\omega 1}$ é uma sequência crescente, $\left(X_{i, \alpha}\right)_{\alpha<\omega_{1}}$ também o é. $\quad \mathcal{C}_{i} \in \bigcup_{\alpha<\omega_{1}} M_{\alpha}$ garante que $\bigcup_{\alpha<\omega_{1}} X_{i, \alpha}=X_{i}$.

Como $\mathcal{C}_{i, \alpha}$ é uma base enumerável para $X_{i, \alpha}$, temos que $X_{i, \alpha}$ é metrizável, pelo Teorema da metrização de Urysohn (veja, por exemplo, [Eng89]). Como supomos $\mathfrak{d}=\mathfrak{b}=\aleph_{1}$, pelo lema 3.3.11, temos que $\nabla_{i \in \omega} X_{i, \alpha}$ é $\omega_{1}$-metrizável para cada $\alpha \in \omega_{1}$. Podemos então construir para cada ponto $x \in \nabla_{i \in \omega} X_{i, \alpha}$ uma base $\mathcal{B}_{\alpha}(x)=\left\{U(x)_{\gamma, \alpha}: \gamma<\omega_{1}\right\}$ satisfazendo as condições da definição 1.2 .30 .

Para cada ponto $x \in \nabla_{i \in \omega} X_{i}$, podemos então definir a base local

$$
\mathcal{B}(x)=\left\{U(x)_{\alpha}=\bigcap_{\beta, \gamma<\alpha} U(x)_{\gamma, \beta}: U_{\gamma, \beta} \in \mathcal{B}_{\beta}(x), \alpha<\omega_{1}\right\} .
$$

Como $\nabla_{i \in \omega} X_{i}$ é P-espaço (lema 3.1.4), temos que os elementos de $\mathcal{B}(x)$ são abertos. As propriedades de cada $\mathcal{B}_{\alpha}(x)$ garantem que $\mathcal{B}(x)$ testemunhe que $\nabla_{i \in \omega} X_{i}$ é $\omega_{1}$-metrizável, como queríamos. 
Os resultados anteriores possuem em comum a hipótese de os fatores serem paracompactos. O seguinte resultado, por Lawrence em [Law88], mostra que é possível obter resultados semelhantes sem precisar que os fatores sejam paracompactos.

Teorema 3.3.13 (Lawrence). Supondo $\mathfrak{b}=\mathfrak{d}$ ou $\mathfrak{d}=\mathfrak{c}$, se $X_{i}$ é um espaço metrizável enumerável, então $\square_{i \in \omega} X_{i}$ é ultraparacompacto.

A ideia da prova deste teorema consiste em usar a hipótese para ordenar o produto nabla de maneira a formar uma árvore, a qual nos auxilie a refinar uma cobertura aberta do produto caixa. Para tanto, Lawrence formulou a Hipótese da Ordem (OH), a qual consiste na existência de tal ordenação do produto nabla. Sua prova consistiu então em duas etapas: mostrar que $\mathfrak{b}=\mathfrak{d}$ ou $\mathfrak{d}=\mathfrak{c}$ implicam em OH, e mostrar que $\mathrm{OH}$ implica que $\square_{i \in \omega} X_{i}$ é ultraparacompacto. Utilizaremos, contudo, uma versão modificada desta demonstração, feita por Roitman em [Roi11], a qual utiliza a mesma ideia.

Definição 3.3.14 (Hipótese da Ordem). Seja $X_{i}$ um espaço métrico enumerável para cada $i \in \omega$. A Hipótese da Ordem $(\mathrm{OH})$ é a seguinte afirmação: existe uma ordem parcial $\preceq$ sobre $\nabla_{i \in \omega} X_{i}$ satisfazendo:

- $\left(\nabla_{i \in \omega} X_{i}, \preceq\right)$ é uma árvore de altura $\leq \mathfrak{d}$;

- $\bigcup\left(p^{\uparrow}\right) \subset \square_{i \in \omega} X_{i}$ é aberto para cada $p \in \nabla_{i \in \omega} X_{i}$.

Como a $\mathrm{OH}$ e os teoremas que a envolvem não consideram a topologia do produto nabla $\nabla_{i \in \omega} X_{i}$, podemos utilizar ao invés um conjunto $Y \subset X$ $={ }^{*}$-transversal, isto é, constituído precisamente por 1 representante de cada classe de equivalência da relação $=^{*}$.

Lema 3.3.15. $\mathfrak{b}=\mathfrak{d}$ implica $O H$.

Demonstração. Começaremos pelo caso $\mathfrak{b}=\mathfrak{d}$. Como cada $X_{i}$ é enumerável, podemos ordenar $X_{i}$ com a ordem de $\omega$ e $\square_{i \in \omega} X_{i}$ com a ordem $\leq^{*}$. Pela proposição 3.3.4(item 3), temos uma b-escala $\left(h_{\alpha}\right)_{\alpha<\mathfrak{b}}$ em $\square_{i \in \omega} X_{i}$.

Para cada $\alpha<\mathfrak{b}$, definimos a função $\gamma_{\alpha}: \omega \longrightarrow \mathbb{R}^{+}$definida por

$$
\gamma_{\alpha}(n)=\inf \left\{d_{n}(a, b): a \leq b \leq h_{\alpha}(n)+1\right\}
$$


A partir de cada $\gamma_{\alpha}$, vamos construir, para cada $\alpha<\mathfrak{b}$, uma espécie de quasi-métrica $^{1} \delta_{\alpha}:\left(\square_{i \in \omega} X_{i} \times \square_{i \in \omega} X_{i}\right) \longrightarrow\left(\mathbb{R}^{+}\right)^{\omega}$ definida por:

$$
\left(\delta_{\alpha}(x, y)\right)_{i}=\delta_{\alpha, i}\left(x_{i}, y_{i}\right)=\frac{d_{n}\left(x_{i}, y_{i}\right)}{\gamma_{\alpha}(i)}
$$

A partir dessa sequência, definimos para cada $x \in \square_{i \in \omega} X_{i}$ e cada $\alpha<\mathfrak{b}$ o conjunto

$$
u_{x, \alpha}=\left\{y \in \square_{i \in \omega} X_{i} \text { : a sequência } \delta_{\alpha}(x, y) \text { converge para } 0\right\} \text {. }
$$

Como $\left(h_{\alpha}\right)_{\alpha<\mathfrak{b}}$ é uma $\mathfrak{b}$-escala, podemos definir $\alpha_{x}$ para cada $x \in \square_{i \in \omega} X_{i}$ como o menor $\alpha<\mathfrak{b}$ tal que $x \leq^{*} h_{\alpha}$. Definimos $u_{x}=u_{x, \alpha_{x}}$, e a partir de tais conjuntos, construiremos a ordem $\preceq$ sobre $\nabla_{i \in \omega} X_{i}$, de maneira que $u_{x}=\bigcup\left(q(x)^{\uparrow}\right)$. Para tanto, definimos a ordem parcial $\preceq$ em $\nabla_{i \in \omega} X_{i}$ por:

$$
q(x) \preceq q(y) \Longleftrightarrow u_{x} \supset u_{y}
$$

Para verificar que $\left(\nabla_{i \in \omega} X_{i}, \preceq\right)$ satisfaz $\mathrm{OH}$, mostraremos que esta construção possui as seguintes propriedades:

I) se $x, y \in \square_{i \in \omega} X_{i}$ são tais que $x={ }^{*} y$, então para cada $\alpha<\mathfrak{b}$ temos $u_{x, \alpha}=u_{y, \alpha}\left(\right.$ consequentemente $q(x) \subset u_{x, \alpha}$ para todo $\left.\alpha<\mathfrak{b}\right)$;

II) se $\alpha_{x}, \alpha_{y} \leq \alpha$ e $q(x) \neq q(y)$, então $u_{x, \alpha} \cap u_{y, \alpha}=\emptyset$;

III) se $\alpha_{x}<\alpha_{y}$, então ou $u_{x} \supset u_{y}$ ou $u_{x} \cap u_{y}=\emptyset$.

Para verificar (I), dados $x={ }^{*} y$, temos que existe $n_{0} \in \omega$ tal que $x_{i}=y_{i}$ para todo $i>n_{0}$. Logo, dado $z \in \square_{i \in \omega} X_{i}$, temos que $\left(\delta_{\alpha}(x, z)\right)_{i}=\left(\delta_{\alpha}(y, z)\right)_{i}$ para todo $i>n_{0}$.

Para verificar (II), sejam $\alpha_{x}, \alpha_{y} \leq \alpha \operatorname{com} q(x) \neq q(y)$. Para todo $n \in \omega$, temos $x_{n}, y_{n}<h_{\alpha}(n)$. Portanto temos $d_{n}\left(x_{n}, y_{n}\right) \geq \gamma_{\alpha}(n)$, e assim $\delta_{\alpha}(x, y)_{n} \geq 1$. A desigualdade triangular garante que $u_{x, \alpha} \cap u_{y, \alpha}=\emptyset$.

\footnotetext{
${ }^{1}$ isto é, uma métrica que não necessariamente satisfaz a simetria.
} 
Para verificar (III), note que fixado $x \in \square_{i \in \omega} X_{i}$, temos $u_{x, \alpha} \supset u_{x, \beta}$ sempre que $\alpha<\beta$. O mesmo ocorre para $y$ fixado. Portanto ou $u_{y, \alpha_{x}}=u_{x, \alpha_{x}}$ ou $u_{y, \alpha_{x}} \cap u_{x, \alpha_{x}}=\emptyset$.

Vamos agora verificar que $\left(\nabla_{i \in \omega} X_{i}, \preceq\right)$ satisfaz a Hipótese da Ordem. A propriedade (I) garante que a relação $\preceq$ está bem definida. As propriedades reflexiva e transitiva são satisfeitas por $\preceq$ devido a $\supset$ também satisfazê-las. Já a propriedade antissimétrica é consequência de (II). Juntos, os itens (II) e (III) mostram que $\left(\nabla_{i \in \omega} X_{i}, \preceq\right)$ é uma árvore, e que $\alpha_{x}$ é altura de $q(x)$. Isto garante também que a altura de $\left(\nabla_{i \in \omega} X_{i}, \preceq\right)$ é menor ou igual a $\mathfrak{b}$.

Por fim, basta verificar que para qualquer $x \in \square_{i \in \omega} X_{i}, u_{x}$ é aberto. Seja $y \in u_{x}$. Para cada $i \in \omega$, definimos $\epsilon_{i}=\left(\delta_{\alpha_{x}}(x, y)\right)_{i}$ e $V_{i}=B_{\delta_{\alpha_{x}, i}}\left(y_{i}, \epsilon_{i}\right)$. Vamos mostrar que $V=\prod_{i \in \omega} V_{i} \subset u_{x}$. Seja $z \in V$. Para cada $i \in \omega$, pela desigualdade triangular, temos:

$$
\left(\delta_{\alpha_{x}}(x, z)\right)_{i} \leq\left(\delta_{\alpha_{x}}(x, y)\right)_{i}+\left(\delta_{\alpha_{x}}(y, z)\right)_{i} \leq 2 \epsilon_{i}
$$

Como $y \in u_{x}$, temos que a sequência $\left(2 \epsilon_{i}\right)_{i \in \omega}$ converge para 0 , e portanto $z \in u_{x}$ como gostaríamos.

Lema 3.3.16. A hipótese da ordem (OH) implica que o produto enumerável de espaços metrizáveis enumeráveis é paracompacto.

Originalmente, Lawrence demonstrou tal resultado refinando cuidadosamente uma cobertura de $\square_{i \in \omega} X_{i}$ em cada nível da árvore obtida por $\mathrm{OH}$. No entanto, posteriormente Wingers demonstra em [Win94] a seguinte generalização do teorema 3.3.13.

Teorema 3.3.17 (Wingers). Suponha $\mathfrak{d}=\mathfrak{c}$. Seja $X_{i}$ um espaço $\sigma$-compacto, zero dimensional, primeiro enumerável com $\left|X_{i}\right| \leq \mathfrak{c}$ para cada $i \in \omega$. Então $\square_{i \in \omega} X_{i}$ é ultraparacompacto.

Além de cobrir o teorema 3.3.13 para o caso $\mathfrak{d}=\mathfrak{c}$, a demonstração de Wingers nos fornece um método mais simples para demonstrar o lema 3.3.16. Contudo, precisaremos de alguns outros resultados de [Win94]. 
Lema 3.3.18. Seja $X_{i}$ um espaço primeiro enumerável para cada $i \in \omega$. Dada uma família $\mathcal{U}=\left\{U_{\alpha}: \alpha<\beta\right\}$ de caixas fechadas de $\square_{i \in \omega} X_{i}$, com $\beta<\mathfrak{d}$, temos que o conjunto $\bigcup\{\bigcup q(U): U \in \mathcal{U}\}$ é fechado.

Demonstração. Seja $x \in \square_{i \in \omega} X_{i} \backslash \bigcup\{\bigcup q(U): U \in \mathcal{U}\}$. Fixamos $\alpha<\beta$ e definimos $S_{\alpha}=\left\{i \in \omega: x_{i} \notin\left(U_{\alpha}\right)_{i}\right\}$. Note que $S_{\alpha}$ é infinito. Construímos então uma vizinhança aberta $V_{\alpha}=\prod_{i \in \omega}\left(V_{\alpha}\right)_{i}$ de $x$ definida por $\left(V_{\alpha}\right)_{i}=$ $X_{i} \backslash\left(U_{\alpha}\right)_{i}$ para $i \in S_{\alpha}$ e $\left(V_{\alpha}\right)_{i}=\left(U_{\alpha}\right)_{i}$ caso contrário.

Para cada $i \in \omega$, seja $\left\{B_{i, j}: j \in \omega\right\}$ uma base local decrescente para $x_{0}$. Para cada $\alpha<\beta$, definimos uma função $f_{\alpha}: \omega \longrightarrow \omega$ dada por $f_{\alpha}(i)=$ $\min \left\{j \in \omega: B_{i, j} \subset\left(V_{\alpha}\right)_{i}\right\}$. Pelo lema 3.3.7, podemos construir uma função $g: \omega \longrightarrow \omega$ tal que o conjunto $\left\{i \in S_{\alpha}: f_{\alpha}(i) \leq g(i)\right\}$ seja infinito para cada $\alpha<\beta$. Definimos a vizinhança $V=\prod_{i \in \omega} B_{i, g(i)}$ de $x$. Para cada $\alpha<\beta$, como o conjunto $\left\{i \in \omega: V_{i} \cap\left(U_{\alpha}\right)_{i}\right\}$ é infinito, temos que $V \cap \bigcup q\left(U_{\alpha}\right)=\emptyset$, como gostaríamos.

Definição 3.3.19. Seja $\mathcal{U}=\left\{U_{\alpha}=\prod_{i \in \omega} U_{\alpha, i}: \forall i \in \omega, U_{\alpha, i} \subset X_{i}\right.$ e $\left.\alpha<\beta\right\}$ uma família qualquer de caixas (não necessariamente abertas) de $\square_{i \in I} X_{i}$.

- Definimos $\mathcal{U}(x, n)=\left\{U \in \mathcal{U}: \forall i \geq n, x_{i} \in U_{i}\right\}$ e $\mathcal{U}(x)=\bigcup_{n \in \omega} \mathcal{U}(x, n)$

- Dizemos que $\mathcal{U}$ é uma família simples se para todo $x \in \square_{i \in \omega} X_{i}$ e todo $n \in \omega$ temos

$$
\mathcal{U}(x, n) \text { é infinito } \Longrightarrow\left\{y \in \square_{i \in \omega} X_{i}: \forall i \geq n, y_{i}=x_{i}\right\} \subset \bigcup \mathcal{U}
$$

- Dizemos que $\mathcal{U}$ é uma família afunilada se $\beta=\omega$ e para todo $j \in$ $\omega, \forall i \geq j, U_{j+1, i}=U_{j, i}$

- Dadas duas caixas $U$ e $V$ em $\square_{i \in \omega} X_{i}$, dizemos que $U$ e $V$ são fortemente disjuntas se $\left\{i \in \omega: U_{i} \cap V_{i}=\emptyset\right\}$ for infinito.

Lema 3.3.20. Seja $\mathcal{U}$ uma família simples de caixas fechadas em $\square_{i \in \omega} X_{i}$, onde $X_{i}$ é primeiro enumerável para todo $i \in \omega$. Se $|\mathcal{U}|<\mathfrak{d}$, então $\bigcup \mathcal{U}$ é fechado.

\footnotetext{
${ }^{2}$ Tapered family, no original.
} 
Demonstração. Seja $x \in \square_{i \in \omega} X_{i} \backslash \bigcup \mathcal{U}$. Vamos construir uma vizinhança $V$ de $x$ satisfazendo as seguintes propriedades:

(i) $\forall i \in \omega, \forall U \in \mathcal{U}(x, i+1) \backslash \mathcal{U}(x, i), V_{i} \cap U_{i}=\emptyset$

(ii) $V$ é fortemente disjunto de cada elemento de $\mathcal{U} \backslash \mathcal{U}(x)$.

Como $\mathcal{U}$ é simples, pela contra-positiva da definição de família simples, temos que $\mathcal{U}(x, i)$ é finito para todo $i \in \omega$. Fixado $i \in \omega$, podemos então escolher uma vizinhança aberta $V_{i}^{\prime}$ de $x_{i}$ tal que para cada $U \in \mathcal{U}(x, i+1) \backslash$ $\mathcal{U}(x, i)$ tenhamos $V_{i}^{\prime} \cap U_{i}=\emptyset$, pois tais $U$ são finitos e satisfazem $x_{i} \notin U_{i}$.

Note que pela definição de $\mathcal{U}(x)$, temos $x \notin \bigcup\{\bigcup q(U): U \in \mathcal{U} \backslash \mathcal{U}(x)\}$. Tal conjunto é fechado (lema 3.3.18), logo podemos escolher uma vizinhança básica aberta $V^{\prime \prime}$ de $x$ tal que $V^{\prime \prime} \cap \bigcup\{\bigcup q(U): U \in \mathcal{U} \backslash \mathcal{U}(x)\}=\emptyset$. Temos que $V^{\prime \prime}$ é fortemente disjunto de cada $U \in \mathcal{U} \backslash \mathcal{U}(x)$. De fato, suponha que $V^{\prime \prime}$ não seja fortemente disjunto de algum $U \in \mathcal{U} \backslash \mathcal{U}(x)$. Dado $p \in V^{\prime \prime}$ tal que $p_{i} \in V_{i}^{\prime \prime} \cap U_{i}$ para todo $i>n$, para algum $n \in \omega$, temos que $q(p) \in q(U)$, $\operatorname{logo} p \in \bigcup q(U)$, o que é um absurdo.

Temos então que $V=V^{\prime} \cap V^{\prime \prime}$ é uma vizinhança de $x$ que satisfaz (i) e (ii). Como $x \notin \cup U$, temos $\mathcal{U}(x, 0)=\emptyset$, de maneira que $\mathcal{U}(x)=$ $\bigcup_{i \in \omega}(\mathcal{U}(x, i+1) \backslash \mathcal{U}(x, i))$. Por (i), temos que $V \cap \bigcup U(x)=\emptyset$, enquanto que por (ii) temos que $V \cap \bigcup(\mathcal{U} \backslash \mathcal{U}(x))=\emptyset$. Assim, $V \cap \bigcup \mathcal{U}=\emptyset$, como gostaríamos.

Temos agora ferramentas suficientes para demonstrar o lema 3.3.16.

Demonstração do lema 3.3.16. Suponhamos OH. Sejam $X_{i}$ enumerável e metrizável para cada $i \in \omega, \preceq$ a ordem sobre $\nabla_{i \in \omega} X_{i}$ obtida por $\mathrm{OH}$ e $u_{p}=$ $\left\{q \in \nabla_{i \in \omega} X_{i}: p \preceq q\right\}$. Seja $\mathcal{W}$ uma cobertura aberta de $\square_{i \in \omega} X_{i}$. Como cada $X_{i}$ é metrizável e enumerável, temos em particular que $X_{i}$ é regular e primeiro enumerável, e portanto zero dimensional (proposição 1.2.8). Portanto $\square_{i \in \omega} X_{i}$ é zero dimensional. Podemos também escrever $X_{i}=\left\{m_{i, j}: j \in \omega\right\}$.

Vamos construir um refinamento $\left\{V_{y, n, l}: y \in \nabla_{i \in \omega} X_{i}, n \in \omega, l<f(l)\right\}$ de $\mathcal{W}$ por indução na altura da árvore $\left(\nabla_{i \in \omega} X_{i}, \preceq\right)$. Para tanto, construiremos 
paralelamente uma função ilimitada $k_{y}: \omega \longrightarrow \omega$ para cada $y \in \nabla_{i \in \omega} X_{i}$. Definimos $V_{y, n}=\bigcup_{l<f(l)} V_{y, n, l}$ e $V_{y}=\left\{V_{y, n}: n \in \omega\right\}$ e vamos escolher um representante $\widehat{y} \in y$. Queremos que, para cada $y \in \nabla_{i \in \omega} X_{i}$, nossa construção satisfaça as seguintes propriedades:

A. $\mathcal{V}_{y}=\bigcup\left\{V_{z}: z \prec y\right\}$ é uma família simples.

B. se $V_{y} \neq \emptyset$, então $V_{y}$ é uma família simples e afunilada.

C. se $\bigcup V_{y} \neq \emptyset$, então $y \subset \bigcup V_{y}$

D. se $V_{y} \neq \emptyset$, então $\widehat{y} \in C_{y, n}=\left(\prod_{i<n}\left\{m_{i, j}: j \leq k_{y}(n)\right\}\right) \times\left(\prod_{i \geq n}\left\{m_{i, j}\right\}\right) \subset$ $V_{y, n} \subset \bigcup u_{y}$.

E. $\forall n \in \omega, k_{y}(n) \geq n$.

F. Para quaisquer $z \in \nabla_{i \in \omega} X_{i}$ e $n \in \omega$ tais que $z \preceq y$ e $V_{n, z} \cap V_{n, y} \neq \emptyset$, temos $k_{z}(n)<k_{y}(n)$.

G. se $V_{y} \neq \emptyset$, então $\widehat{y} \notin \bigcup \mathcal{V}_{y}$.

H. Se $V_{y} \neq \emptyset$, então $\forall i \in \omega, \forall U \in \mathcal{V}_{y}(\widehat{y}, i+1) \backslash \mathcal{V}_{y}(\widehat{y}, i),\left(V_{y, 0}\right)_{i} \cap U_{i}=\emptyset$

I. Se $V_{y} \neq \emptyset$, então $V_{y, 0}$ é fortemente disjunto dos elementos de $\mathcal{V}_{y} \backslash \mathcal{V}_{y}(\widehat{y})$.

Sejam $n \in \omega$ e $y \in \nabla_{i \in \omega} X_{i}$ cuja altura é 0. Fixemos $\widehat{y} \in \square_{i \in \omega} X_{i}$ um representante da classe $y$. Definimos $k_{y}(n)=j(\widehat{y}, n)+n$ para cada $n \in \omega$, onde $j(x, l)$ é o número natural tal que $x_{l}=m_{j(x, l)}$.

Vamos construir $V_{y, n}$ satisfazendo D. Para $n=0$, como temos $C_{y, 0}=\{\widehat{y}\}$, podemos escolher um aberto fechado básico $V^{\prime}$ tal que $\widehat{y} \in V^{\prime} \subset W \cap \bigcup u_{y}$ para algum $W \in \mathcal{W}$. Definimos assim $V_{y, 0}=V^{\prime}$. Para $n>0$, note que $C_{y, n}$ é finito. Então para cada $c \in C_{y, n}$, podemos escolher um aberto fechado básico $V_{c}$ tal que $c \in V_{c} \subset W_{c} \cap \bigcup u_{y}$ para algum $W_{c} \in \mathcal{W}$. Definimos então $V_{y, n}=\left(\prod_{i<n} \bigcup_{c \in C_{y, n}}\left(V_{c}\right)_{i}\right) \times\left(\prod_{i \geq n} V_{i}^{\prime}\right)$.

Para cada $n \in \omega$, como $C_{y, n}$ é compacto, podemos escolher finitos pontos $c_{l} \in C_{y, n}$, com $l<f(n)$ para algum $f(l)<\omega$, de maneira que $V_{y, n} \subset$ $\bigcup_{l<f(n)} V_{c_{l}}$. Definimos então $V_{y, n, l}=\left(\prod_{i<n}\left(V_{c}\right)_{i}\right) \times\left(\prod_{i \geq n}\left(V_{c}\right)_{i} \cap V_{i}^{\prime}\right)$. Assim, 
temos $V_{y, n, l} \in W_{c_{l}} \in \mathcal{W}$ e $V_{y, n}=\bigcup_{l<f(l)} V_{y, n, l}$. De fato, por um lado já temos que $V_{y, n} \supset \bigcup_{l<f(n)} V_{y, n, l}$, uma vez que $\forall i \in \omega, \forall l<f(l),\left(V_{y, n, l}\right)_{i} \subset\left(V_{y, n}\right)_{i}$. Por outro lado, seja $x \in V_{y, n}$. Como $\left\{V_{c_{l}}: l<f(n)\right\}$ cobre $V_{y, n}$, temos que existe $x \in V_{c_{l}}$ para algum $l<f(n)$. Como $\forall i \geq n, x_{i} \in V^{\prime}$, temos que $x \in V_{y, n, l}$, como gostaríamos.

Temos que a família $V_{y}=\left\{V_{y, n}: n \in \omega\right\}$ é afunilada e satisfaz $y=$ $\bigcup_{n \in \omega} C_{y, n} \subset V_{y}$. Temos também que a mesma é simples. De fato, sejam $p \in \square_{i \in \omega} X_{i}$ e $m \in \omega$, e suponha $V_{y}(p, m)$ infinito. Seja $q \in \square_{i \in \omega} X_{i}$ tal que $\forall i \geq m, q_{i}=p_{i}$. Definimos $a=\max \{j(q, i): i<m\}$. Como $k_{y}$ é ilimitado, podemos encontrar $b \in \omega$ tal que $k_{y}(b)>a$. Em seguida, como supomos $V_{y}(p, m)$ infinito, existe algum $c>b$ tal que $V_{y, c} \in V_{y}(p, m)$. Temos que $q \in C(y, c) \subset \bigcup V_{y}$, e , portanto, $V_{y}$ é de fato simples.

Agora fixemos $y \in \nabla_{i \in \omega} X_{i}$ cuja altura é $0<\alpha<\mathfrak{d}$. Suponha $k_{z}$ e $V_{z}$ construídos para cada $z \prec y$. O próximo passo na construção é puramente técnico e não acrescenta muito conceitualmente, de modo que o omitiremos. Detalhes da construção passo a passo estão na demonstração do Lema 13 em [Win94]. As condições G,H e I são utilizadas somente nesta afirmação.

Afirmação. Podemos construir uma função $k_{y}: \omega \longrightarrow \omega$ satisfazendo as condições E e F.

Com $k_{y}$ construído, vamos escolher $\widehat{y}$. Se $y \subset \bigcup_{z \prec y}\left(\bigcup V_{y}\right)$, podemos definir $f(n)=1$ e $V_{y, n, 0}=\emptyset$ para todo $n \in \omega$ e escolher $\widehat{y} \in y$ um representante qualquer. Caso contrário, escolhemos um representante $\widehat{y} \in y$ tal que $\widehat{y} \notin \bigcup_{z \prec y}\left(\bigcup V_{y}\right)$. Podemos construir, portanto, do mesmo modo que na demonstração do lema 3.3.20, um aberto fechado básico $V$ tal que $\widehat{y} \in V \subset W \cap \bigcup\left(u_{y}\right)$, para algum $W \in \mathcal{W}$, de modo que $V$ é fortemente disjunto de $\left\{U \in \bigcup_{z \prec y} V_{y}: \forall n \in \omega, \exists i \geq n, x_{i} \notin U_{i}\right\}$. Definimos então $V_{y, 0,0}=V$ e $f(0)=1$, de maneira que $V_{y, 0}=V$. De tal forma, temos satisfeitas as condições H e I. Para $n>0$, construímos $V_{y, n}$ e $V_{y, n, l}$ como anteriormente.

Novamente temos as condições B até D satisfeitas. Vamos mostrar agora que a propriedade $\mathrm{F}$ implica a propriedade $\mathrm{A}$.

Seja $p \in \square_{i \in \omega} X_{i}$ e $n \in \omega$. Suponhamos $\mathcal{V}_{y}(p, n)$ infinito. Definimos 
$J=\left\{j \in \omega: \exists z \prec y, V_{z, j} \in \mathcal{V}_{y}(p, n)\right\}$. Seja $q \in \square_{i \in \omega} X_{i}$ tal que $\forall i \geq n, q_{i}=$ $p_{i}$. Como anteriormente, definimos $a=\max \{j(q, i): i<m\}$. O objetivo é encontrar algum $V \in \mathcal{V}_{y}$ tal que $q \in V$. Dividimos então a demonstração em 3 casos:

1. Suponha $J$ infinito. Podemos encontrar $j \in J$ tal que $j>a$. Seja $z \prec y$ tal que $V_{z, j} \in \mathcal{V}_{y}(p, n)$. Pela condição $\mathrm{E}$, temos que $k_{z}(j) \geq j>a$. Temos então que $q \in V_{z, j}$, como gostaríamos.

2. Suponha $J$ finito. Como supomos $\mathcal{V}_{y}(p, n)$ infinito, pelo princípio da casa dos pombos, existe algum $j_{0} \in J$ para o qual existem infinitos $z \prec y$ tais que $V_{z, j_{0}} \in \mathcal{V}_{y}(p, n)$. Suponhamos $j_{0} \geq n$. Denotemos $Z=\left\{z \prec y: V_{z, j_{0}} \in \mathcal{V}_{y}(p, n)\right\}$. Seja $r \in \square_{i \in \omega} X_{i}$ dado por $r_{i}=m_{i, 0}$ para $i<n$ e $r_{i}=p_{i}$ para $i \geq n$. Note que, para todo $z \in Z$, temos $r \in C_{z, j_{0}} \subset V_{z, j_{0}}, \log \bigcap_{z \in Z} V_{z, j_{0}} \neq \emptyset$. Pela condição $\mathrm{F}$, temos que a sequência $\left(k_{z}\left(j_{0}\right)\right)_{z \in Z}$ ordenada por $\prec$ é crescente. Logo, podemos encontrar $z \in Z$ tal que $k_{z}\left(j_{0}\right)>a$. Temos então que $q \in V_{z, j_{0}}$, como gostaríamos.

3. Suponhamos $J$ finito e $j_{0}<n$. Pela condição B, para cada $z \in Z$, temos que $V_{z}$ é afunilada. Portanto, temos que $\forall i \geq n,\left(V_{z, n}\right)_{i}=\left(V_{z, j_{0}}\right)_{i}$, de maneira que $x_{i} \in\left(V_{z, n}\right)_{i}$. Desta maneira, $V_{z, n} \in \mathcal{V}_{y}(p, n)$ para infinitos $z \prec y$. Assim, podemos proceder como no item anterior, apenas substituindo $j_{0}$ por $n$, e encontrar $z \prec y$ tal que $q \in V_{z, n}$, como gostaríamos.

Vamos então construir um refinamento $\mathcal{U}$ aberto dois a dois disjunto de $\mathcal{W}$. Definimos os elementos de $\mathcal{U}$ por

$$
U_{y, n, l}=V_{y, n, l} \backslash\left(\bigcup \mathcal{V}_{y} \cup \bigcup_{i<n} V_{y, i} \cup \bigcup_{i<l} V_{y, n, i}\right) .
$$

Como cada $V_{y}$ cobre $y$, temos que tal refinamento de fato cobre $\square_{i \in \omega} X_{i}$. Note que a condição D garante que $\bigcup V_{y} \subset \bigcup u_{y}$, portanto $y \preceq q(x)$. Assim, podemos bem ordenar $A_{x}$ pela ordem lexicográfica, de maneira que o único 
elemento de $\mathcal{U}$ ao qual $x$ pertence é $V_{\min A_{x}}$. Por fim, o lema 3.3 .20 garante que cada $U_{y, n, l}$ é aberto, completando a demonstração.

\subsection{Produtos de ordinais}

Uma das questões recorrentes sobre a paracompacidade dos produtos caixa diz respeito ao produto caixa de ordinais. Sobre o assunto, podemos citar [Rud74], [Wil77a], [YW87] e [Wil77b]. A principal questão trata de encontrar hipóteses que impliquem a paracompacidade de $\square^{\omega}(\omega+1)$. Tal questão motivou o estudo de produtos caixa de ordinais compactos.

Veremos um resultado introduzido por Kunen em [Kun78] que serve como ferramenta para estudar a paracompacidade de produtos de ordinais. Para isto, utilizaremos o conceito de espaço disperso. Com este resultado, temos que $\mathrm{CH}$ é suficiente para que $\square^{\omega}(\omega+1)$ seja paracompacto.

Teorema 3.4.1 (Kunen). Se para cada $i \in \omega, X_{i}$ é um espaço compacto Hausdorff e disperso, então $\square_{i \in \omega} X_{i}$ é c-Lindelöf.

Demonstração. Seja $\mathcal{U}$ uma cobertura aberta de $\square_{i \in \omega} X_{i}$. Vamos construir um refinamento fechado de $\mathcal{U}$ de cardinalidade $\leq \mathfrak{c}$. Para isso, vamos construir por indução uma árvore de altura $\omega_{1}$ de subconjuntos fechados de $\square_{i \in \omega} X_{i}$.

Denotamos por $T=\mathfrak{c}^{<\omega_{1}}=\bigcup_{\alpha<\omega_{1}} \mathfrak{c}^{\alpha}$. Dados $\alpha \in \mathfrak{c}$ e $t \in T$, definimos a composição $t \smile \alpha \in T$ como a função parcial dada por $\operatorname{dom}(t \smile \alpha)=\operatorname{dom}(t)+1$, $t \alpha \Upsilon_{\operatorname{dom}}(t)=t$ e $t \smile \alpha(\operatorname{dom}(t))=\alpha$.

Para cada $t \in T$, vamos construir uma caixa fechada $K(t)=\prod_{i \in \omega} K_{i}(t)$ satisfazendo:

1. $K(t)=\bigcup_{\alpha<\mathfrak{c}} K(t \smile \alpha)$

2. se $\operatorname{dom}(t)$ for um ordinal limite, então $K(t)=\bigcap_{\beta<\operatorname{dom}(t)} K(t\lceil\beta)$

3. para cada $\alpha<\mathfrak{c}, K(t \smile \alpha)$ satisfaz uma e somente uma das seguintes condições: 
(a) $\exists U \in \mathcal{U}, K(t \smile \alpha) \subset U$

(b) $\exists n \in \omega, \operatorname{rank}\left(K_{n}(t \smile \alpha)\right)<\operatorname{rank}\left(K_{n}(t)\right)$

Começamos definindo $K(0)=\square_{i \in \omega} X_{i}$, onde 0 representa a função nula. Fixemos $t \in T$ e suponhamos $K(t)$ já construído. Para cada $i \in \omega$, definimos

$$
\beta_{i}=\operatorname{rank}\left(K_{i}(t)\right) \text { e } Y_{i}=\left(K_{i}(t)\right)^{\left(\beta_{i}\right)} .
$$

Pela observação 1.2.15, temos que $Y_{i}$ é finito e não vazio. Note que $\left|\prod_{i \in \omega} Y_{i}\right| \leq$ c. Como $\square_{i \in \omega} X_{i}$ é regular, podemos encontrar para cada $y \in \prod_{i \in \omega} Y_{i}$ um aberto $V(y)=\prod_{i \in \omega} V_{i}(y)$ tal que $y \in V(y) \subset \overline{V(y)} \subset U$, para algum $U \in \mathcal{U}$. Definimos então as famílias $\mathcal{V}$ de todos os $V(y)$ para $y \in \prod_{i \in \omega} Y_{i}$ e $\mathcal{K}$ de todas as caixas fechadas $K=\prod_{i \in \omega} K_{i}$ que satisfazem uma e somente uma das seguintes condições:

(i) $\exists V \in \mathcal{V}, K=\bar{V} \cap K(t)$

(ii) $\exists n \in \omega,\left\{\begin{array}{l}\exists \mathcal{V}^{\prime} \in[\mathcal{V}]^{<\omega}, Y_{n} \subset \bigcup_{V \in \mathcal{V}^{\prime}} V_{n} \text { e } K_{n}=K_{n}(t) \backslash \bigcup_{V \in \mathcal{V}^{\prime}} V_{n} \\ \forall i \neq n, K_{i}=K_{i}(t)\end{array}\right.$

Note que, como $|\mathcal{V}| \leq\left|[\mathcal{V}]^{<\omega}\right| \mathfrak{c}$, temos que $|\mathcal{K}| \leq \mathfrak{c}$. Podemos então escolher de maneira sobrejetiva um $K\left(t_{\smile} \alpha\right) \in \mathcal{K}$ para cada $\alpha \in \mathfrak{c}$. Note que as caixas escolhidas na família $\mathcal{K}$ garantem a satisfação do item 3. De fato, se $K \in \mathcal{K}$ satisfizer a condição (i), então $K(t \smile \alpha)=K$ satisfaz a condição (a). Se $K^{\prime}$ satisfaz a condição (ii), então temos $\left(K_{n}^{\prime}\right)^{\left(\beta_{n}\right)}=\emptyset$, e portanto $\operatorname{rank}\left(K_{n}^{\prime}\right)<\beta_{n}$, do que se conclui que $K\left(t \_\alpha\right)=K^{\prime}$ satisfaz a condição (b).

Por fim, para $t \in T$ tal que $\operatorname{dom}(t)$ é um ordinal limite, definimos $K(t)$ como no item 2.

Já mostramos que a árvore construída satisfaz a condição 3 e 2. Falta mostrar que satisfaz a condição 1 . Seja $p \in K(t)$ para algum $t \in T$, e suponha que $p$ não pertença a nenhuma caixa $K \in \mathcal{K}$ satisfazendo a condição ii. Então para cada $i \in \omega$, podemos encontrar $q_{i} \in Y_{i}$ tal que para todo $V \in \mathcal{V}$ temos $q_{i} \in V_{i} \Rightarrow p_{i} \in V_{i}$, pois $V_{i}$ é finito. Considere $q=\left(q_{i}\right)_{i \in \omega} \in \prod_{i \in \omega} Y_{i}$. Logo para todo $V \in \mathcal{V}$, temos $q \in V \Rightarrow p \in V$, $\log p \in \overline{V(q)} \cap K(t) \in \mathcal{K}$, como gostaríamos. 
Construída a árvore como desejamos, vamos mostrar que para qualquer $x \in \square_{i \in \omega} X_{i}$, existe um $t \in T$ e um aberto $U_{t} \in \mathcal{U}$ tal que $p \in K(t) \subset U_{t}$, os quais vão determinar a subcobertura desejada de $\mathcal{U}$, dado que $|T| \leq \mathfrak{c}$. Fixado tal $x \in \square_{i \in \omega} X_{i}$, as condições 1 e 2 nos permitem construir uma função $f: \omega_{1} \longleftrightarrow \mathfrak{c}$ tal que para cada $\gamma \in \omega_{1}$ temos $x \in K(f\lceil\gamma)$. Dado $n \in \omega$, considere a sequência $\left(\beta_{\gamma}(n)\right)_{\gamma \in \omega_{1}}$ dada por $\beta_{\gamma}(n)=\operatorname{rank}\left(K_{n}(f\lceil\gamma))\right.$. A condição 3 junto com a condição 2 garantem que $\left(\beta_{\gamma}(n)\right)_{\gamma \in \omega_{1}}$ seja uma sequência decrescente de ordinais e, portanto, eventualmente constante.

Para cada $n \in \omega$, seja $\delta_{n}$ tal que $\left(\beta_{\gamma}(n)\right)_{\gamma \in \omega_{1}}$ é constante para $\gamma>\delta_{n}$. Como $\operatorname{cf}\left(\omega_{1}\right)>\aleph_{0}$, podemos encontrar $\delta=\max _{n \in \omega} \delta_{n}$. Fixamos $t=f\lceil\delta$. Como para todo $n \in \omega$ temos $\left(\beta_{\gamma}(n)\right)_{\gamma \in \omega_{1}}$ constante para $\gamma>\delta$, pela condição 3 temos que existe $U_{t} \in \mathcal{U}$ tal que $K(t) \subset U_{t}$, como queríamos.

Como todo ordinal sucessor é compacto, junto com o lema 3.1.4 e o teorema 3.1.5, temos o seguinte corolário:

Corolário 3.4.2. Supondo CH, o produto enumerável de ordinais sucessores é paracompacto.

Outras hipóteses mais fracas também implicam na paracompacidade de $\square^{\omega}(\omega+1)$. Por exemplo, em [Wil77b], Williams demonstra que a existência de uma $\lambda$ escala é suficiente para o mesmo resultado.

\subsection{O caso não enumerável}

A maioria dos resultados conhecidos sobre a paracompacidade de produtos caixa se referem a produtos de uma quantidade enumerável de espaços. Pouco se sabe sobre produtos de uma quantidade maior de fatores. Em [RW90], Judith Roitman e Scott Williams apresentam, como diz o título do artigo, alguns resultados sobre a paracompacidade de espaços relacionados a produtos caixa não enumeráveis, como por exemplo seus respectivos produtos nabla.

Lembrando da observação 3.1.2, podemos generalizar o produto nabla de maneira semelhante ao que se faz com o produto caixa ao se definir o $\kappa$ produto caixa. Ao invés de usar o filtro dos cofinitos, usaremos o filtro dos 
coenumeráveis. Podemos também, teoricamente, trabalhar com cardinalidades ainda maiores, visto que para qualquer cardinal infinito $\kappa$, o conjunto $\mathcal{F}_{\kappa}=\{J \in \mathcal{P}(I):|I \backslash J|<\kappa\}$ é um filtro. Contudo, focaremos apenas no caso dos coenumeráveis.

Definição 3.5.1. Seja $X_{i}$ um espaço topológico para cada $i \in I$. Definimos a seguinte relação $\sim$ de equivalência em $\square_{i \in I} X_{i}$ : Dados $x, y \in \square_{i \in I} X_{i}$, definimos:

$$
x \sim y \Longleftrightarrow\left\{i \in I: x_{i} \neq y_{i}\right\} \text { é enumerável. }
$$

Definimos então

$$
\nabla_{i \in I}^{*} X_{i}=\frac{\square_{i \in I} X_{i}}{\sim}
$$

equipado com a topologia quociente. Definimos também a projeção usual $q^{*}: \square_{i \in I} X_{i} \longrightarrow \nabla_{i \in I}^{*} X_{i}$.

A seguinte proposição estabelece condições para a projeção de caixas fechadas serem fechadas. Tal proposição servirá de ferramenta para os demais resultados.

Proposição 3.5.2. Seja $\lambda$ um ordinal. Para cada $\alpha<\lambda$, seja $K_{\alpha}=$ $\prod_{i \in I} K_{\alpha, i}$ uma caixa fechada em $\square_{i \in I} X_{i}$. Então:

1. se $\lambda \leq \omega$, então $\bigcup_{\alpha<\lambda} q\left(K_{\alpha}\right)$ é fechado em $\nabla_{i \in I} X_{i}$.

2. se $\lambda \leq \omega_{1}$, então $\bigcup_{\alpha<\lambda} q^{*}\left(K_{\alpha}\right)$ é fechado em $\nabla_{i \in I}^{*} X_{i}$.

Demonstração. Vamos provar ambos os itens simultaneamente. Seja $K=$ $\bigcup_{\alpha<\lambda} q\left(K_{\alpha}\right)$ (respectivamente $\bigcup_{\alpha<\lambda} q^{*}\left(K_{\alpha}\right)$ ). Seja $y \in \square_{i \in I} X_{i}$ tal que $q(y) \notin$ $K$ (respectivamente, $q^{*}(y) \notin K$ ). Para cada $\alpha \in \lambda$, podemos encontrar $I_{\alpha} \subset I$ tal que $\left|I_{\alpha}\right|=\lambda$ e para qualquer $i \in I_{\alpha}, y_{i} \notin K_{\alpha, i}$. Podemos escolher tais $I_{\alpha}$ de maneira que sejam dois a dois disjuntos. Podemos então escolher uma vizinhança aberta $V=\prod_{i \in I} V_{i}$ de $y$ tal que para cada $\alpha \in \lambda$ e cada $i \in I_{\alpha}$ temos $V_{i} \cap K_{\alpha, i}=\emptyset$.

Observação 3.5.3. Em [vD80], van Douwen usa uma demonstração similar para demonstrar tal resultado para valores maiores de $\lambda$. Primeiramente, ele 
define o produto nabla de maneira mais geral, semelhante à definição 3.5.1, porém criando uma relação de equivalência que associa pontos que coincidem em menos que $|I|$ coordenadas. Com tal definição, a proposição anterior é válida para qualquer $\lambda \leq|I|$.

Diferentemente do lema 3.1.4, se $|I|>\aleph_{0}$, não necessariamente temos que $\nabla_{i \in I} X_{i}$ é um P-espaço. Contudo, supondo a normalidade dos fatores, temos que $\nabla_{i \in I} X_{i}$ e $\nabla_{i \in I}^{*} X_{i}$ são zero dimensionais.

Proposição 3.5.4. Sejam $X_{i}$ espaços topológicos normais para cada $i \in I$. Então os espaços $\nabla_{i \in I} X_{i}$ e $\nabla_{i \in I}^{*} X_{i}$ são zero dimensionais.

Demonstração. Sejam $x \in \square_{i \in I} X_{i}$ e $U=\prod_{i \in I} V_{i}$ uma vizinhança aberta básica de $x$. Para cada $i \in I$, como $X_{i}$ é normal, podemos construir uma sequência $\left(V_{\alpha, i}\right)_{\alpha \in \omega}$ de vizinhanças abertas de $x_{i}$ tal que, para cada $\alpha \in \omega$ temos:

$$
x_{i} \in V_{\alpha, i} \subset \overline{V_{\alpha, i}} \subset V_{\alpha+1, i} \subset U_{i}
$$

Definimos $V_{\alpha}=\prod_{i \in I} V_{\alpha, i}$ para cada $\alpha \in \lambda$. Temos que $\overline{V_{\alpha}} \subset V_{\alpha+1}$. Definimos então $G=\bigcup_{\alpha \in \omega} q\left(V_{\alpha}\right)$ e $G^{*}=\bigcup_{\alpha \in \omega} q^{*}\left(V_{\alpha}\right)$. Temos então que $q(x) \in G \subset q(U)$ e $q^{*}(x) \in G^{*} \subset q^{*}(U)$. $G$ e $G^{*}$ são claramente abertos, enquanto que pela proposição 3.5.2, os mesmos também são fechados. Logo $\nabla_{i \in I} X_{i}$ e $\nabla_{i \in I}^{*} X_{i}$ são zero dimensionais, como gostaríamos.

Com tais proposições, conseguimos o seguinte resultado:

Teorema 3.5.5. Assumindo $2^{\aleph_{1}}=\aleph_{2}$, se $X_{i}$ é um espaço topológico com $\left|X_{i}\right| \leq \aleph_{2}$ para cada $i \in I,|I|=\omega_{1}$, então $\nabla_{i \in I}^{*} X_{i}$ é ultraparacompacto.

Demonstração. Pela hipótese, temos que $\left|\square_{i \in I} X_{i}\right| \leq \omega_{2}$. Podemos então escrever $\nabla_{i \in I}^{*} X_{i}=\left\{q^{*}\left(x_{\alpha}\right): \alpha \in \omega_{2}\right\}$. Seja $\mathcal{U}$ uma cobertura aberta de $\nabla_{i \in I}^{*} X_{i}$. Vamos construir um refinamento $\mathcal{U}^{\prime}=\left\{U_{\alpha}: \alpha \in \omega_{2}\right\}$ de $\mathcal{U}$ dois a dois disjunto, utilizando indução. Suponhamos que $\mathcal{U}_{\alpha}^{\prime}=\left\{U_{\beta}: \beta<\alpha\right\}$ tenha sido construído satisfazendo:

1. todos os elementos de $\mathcal{U}_{\alpha}^{\prime}$ são abertos-fechados e dois a dois disjuntos.

2. todo elemento de $\mathcal{U}_{\alpha}^{\prime}$ está contido em algum elemento de $\mathcal{U}$. 
3. $\mathcal{U}_{\alpha}^{\prime}$ cobre o conjunto $\left\{q^{*}\left(x_{\beta}\right): \beta<\alpha\right\}$.

Se $q^{*}\left(x_{\alpha}\right) \in \bigcup \mathcal{U}_{\alpha}^{\prime}$, escolhemos $U_{\alpha}=U_{\xi}$, com $\xi<\alpha$ qualquer. Caso contrário, como $|\alpha|<\aleph_{2}$, pela proposição 3.5.2, temos que $\bigcup \mathcal{U}_{\alpha}^{\prime}$ é fechado. Logo, pela proposição 3.5.4, podemos achar um aberto fechado que contém $q^{*}\left(x_{\alpha}\right)$ e que não intersecciona $\bigcup \mathcal{U}_{\alpha}^{\prime}$. Definimos então $U_{\alpha}$ como tal aberto, o que completa a indução.

Observação 3.5.6. Note que para os fatores $X_{i}$ satisfazerem as hipóteses do teorema anterior, basta que sejam espaços de Lindelöff, Hausdorff e primeiro enumeráveis, pois pelo Teorema de Arhangelskii (teorema 1.2.21)teríamos $\left|X_{i}\right| \leq 2^{\omega} \leq 2^{\omega_{1}}$

Observação 3.5.7. Novamente em [vD80], van Douwen generaliza este teorema para cardinalidades maiores. Utilizando sua definição de produto nabla (observação 3.5.3), e usando como hipótese $2^{\kappa}=\kappa^{+}$, para $\kappa=|I|$, pode-se generalizar o teorema 3.5.5. Mais do que isso, com tal hipótese van Douwen generaliza inclusive o teorema 3.2.2.

\subsection{O problema dos produtos caixa nos dias de hoje}

A maioria dos resultados neste tópico foram obtidos entre o fim dos anos 70 e começo dos anos 90. Depois disso, poucos resultados novos foram obtidos. No entanto, recentemente o assunto está voltando à tona. Em [Roi07] e [Roi09], Roitman estuda subespaços paracompactos de $\square^{\omega}(\omega+1)$ e $\nabla^{\omega}(\omega+1)$.

Em [Roi11], Roitman cita as seguintes questões que ainda permanecem abertas.

Problema 1. Sem hipóteses extra de consistência, é paracompacto o produto $\square_{i \in \omega} X_{i}$ supondo

- $X_{i}$ compacto e disperso para todo $i \in \omega$ ? 
- $X_{i} \sigma$-compacto e primeiro enumerável para todo $i \in \omega$ ?

- $X_{i}$ compacto e primeiro enumerável para todo $i \in \omega$ ?

- $X_{i}$ compacto e metrizável para todo $i \in \omega$ ?

- $X_{i}=\omega+1$ para todo $i \in \omega$ ?

Problema 2. A paracompacidade implica a paracompacidade hereditária, no caso dos produto nabla enumerável de espaços compactos primeiro enumeráveis?

Devido à relação entre produtos caixa e uniformidades explorada em [Wil84], Williams introduziu nos simpósios de Praga de 2001 e 2006 o conceito de produto caixa uniforme. Tal produto, assim como o $\kappa$-produto caixa, é um intermediário entre o produto de Tychonoff e o produto caixa. A definição de uniformidade e produto caixa uniforme podem ser encontradas no apêndice. A pergunta principal continua a mesma que com o produto caixa: sob quais hipóteses sobre $X$ temos o produto caixa uniforme paracompacto? Bell estuda esta questão em [Bel11] e [Bel14]. 


\section{Capítulo 4}

\section{Aplicações}

Como vimos no capítulo 2, o produto de dois espaços topológicos normais não necessariamente é normal. A partir de tal fato, surge a pergunta: "Sob quais condições o produto de espaços normais é ou não é normal?". Tal questão gerou vários estudos em topologia, dos quais podemos citar [Prz84].

No âmbito de tal pergunta, através do produto caixa podemos construir alguns exemplos de espaços normais cujo produto não é normal. Neste capítulo, apresentamos dois desses exemplos. Na seção 4.1, estudaremos o espaço de Dowker construído por M. E. Rudin em [Rud71]. Na seção 4.2, apresentamos um exemplo construído por J. E. Vaughan [Vau75] de dois espaços $\kappa$-metrizáveis cujo produto não é normal.

\subsection{Espaço de Dowker}

Definição 4.1.1. Um espaço topológico $X$ é um espaço de Dowker se $X$ é normal, porém $X \times \mathbb{I}$ não é normal.

Segundo Mary Ellen Rudin em [Rud84], o interesse por tal espaço nasceu de um teorema de extensão homotópica da topologia algébrica. Tal teorema tinha como hipótese que um determinado espaço topológico $X$ fosse tal que $X \times \mathbb{I}$ fosse normal. Tal hipótese faz sentido em um teorema sobre homotopias, dado que uma homotopia em $X$ é uma função contínua de $X \times \mathbb{I}$ em $X$. Dessa maneira, é natural perguntar se isso significa mais do que dizer que 
$X$ é normal. Então, Hugh Dowker provou em seu artigo [Dow51] que se $X$ é normal, então $X \times \mathbb{I}$ é normal se e somente se $X$ é enumeravelmente paracompacto (teorema 4.1.3).

Definição 4.1.2. Um espaço topológico $X$ é dito enumeravelmente paracompacto se toda cobertura aberta enumerável de $X$ possui um refinamento aberto localmente finito.

Teorema 4.1.3 (Teorema de Dowker). Seja X um espaço normal. Então são equivalentes:

- $X$ é enumeravelmente paracompacto.

- $X \times \mathbb{I}$ é normal.

- Dada uma sequência decrescente de fechados $\left(F_{n}\right)_{n \in \omega}$ em $X$ com interseção vazia, existe uma sequência de abertos $\left(A_{n}\right)_{n \in \omega}$ de abertos em $X$ com interseção vazia tal que $F_{n} \subset A_{n}$ para cada $n \in \omega$.

Portanto, um espaço é de Dowker se e somente se ele é normal e não satisfaz alguma (logo todas) das condições do teorema 4.1.3.

Em [Rud55], Rudin demonstra que a existência de uma reta de Souslin implica a existência de um espaço de Dowker. Nos anos 60, Solovay e Tennenbaum [ST71] demonstraram que a existência de uma reta de Souslin é independente de ZFC, do que se conclui o que a existência de um espaço de Dowker é consistente com ZFC. Posteriormente, Rudin construiu em [Rud71] um espaço de Dowker em ZFC sem nenhuma hipótese adicional, utilizando o produto caixa. Para este espaço, usaremos o produto caixa com a notação de ordem, como explicado na observação a seguir.

Observação 4.1.4. Para cada $i \in I$, seja $X_{i}$ um conjunto equipado com uma ordem $\leq_{i}$ e sua topologia da ordem $\tau_{\leq_{i}}$.

As ordens $\leq_{i}$ induzem uma ordem sobre o produto $\prod_{i \in I} X_{i}$ definida por

$$
x \leq y \Leftrightarrow \forall i \in I, x_{i} \leq y_{i}
$$

para quaisquer $x, y \in \prod_{i \in I} X_{i}$. 
Da mesma maneira, também para quaisquer $x, y \in \prod_{i \in I} X_{i}$, definimos

$$
x<y \Leftrightarrow \forall i \in I, x_{i}<y_{i}
$$

Note que, neste caso, $<$ não corresponde à versão estrita da ordem $\leq$.

Notemos que, com tal ordem, a topologia da ordem $\tau_{\leq}$coincide com a topologia da caixa $\tau_{\square}$. De fato, dados dos pontos $f, g \in \prod_{i \in I} X_{i}$, o intervalo aberto $] f, g\left[\right.$ na ordem < nada mais é do que a caixa aberta $\left.\prod_{i \in I}\right] f_{i}, g_{i}[$.

Exemplo 4.1.5 (O espaço de Dowker de M. E. Rudin). Seja F o espaço topológico $F=\square_{i \in \mathbb{N}^{*}}\left(\omega_{i}+1\right)$. Considere o subespaço

$$
X=\left\{x \in F: \exists k \in \mathbb{N}^{*}, \forall i \in \mathbb{N}^{*}, \omega_{1} \leq \operatorname{cf}\left(x_{i}\right)<\omega_{k}\right\}
$$

Com a topologia de subespaço herdada de $F$, o espaço $X$ é um espaço de Dowker, como iremos demonstrar. $X$ possui também outras propriedades interessantes, apresentadas por Hart em [Har81] e [Har82]. Por exemplo, Hart mostra que $X$ é fortemente coletivamente normal e ortocompacto.

Mostraremos que o espaço $X$ é coletivamente normal (definição 1.2.11), utilizando a demonstração original de M. E. Rudin. Para tal fim, necessitamos do seguinte lema.

Lema 4.1.6. O espaço $X$ do exemplo 4.1.5 é um P-espaço.

Demonstração. Seja $\left(V_{n}\right)_{n \in \omega}$ uma sequência de abertos de $X$. Fixemos $x \in$ $\bigcap_{n \in \omega} V_{n}$. Para cada $n \in \omega$, existe uma caixa aberta $B_{n}$ tal que $x \in B_{n} \subset V_{n}$. Como visto na observação 4.1.4, podemos escrever $\left.B_{n}=\right] g_{n}, f_{n}[\cap X$, com $g_{n}, f_{n} \in F$ e considerando a ordem $\leq$ sobre $F$. Definimos então $g \in F$ dado por $g_{i}=\sup _{n \in \omega}\left(g_{n}\right)_{i}$. Note que $g \leq x$. Contudo, para cada $i \in \mathbb{N}^{*}$, temos $\operatorname{cf}\left(x_{i}\right) \geq \omega_{1}$, pois $x \in X \operatorname{e~} \operatorname{cf}\left(g_{i}\right) \leq \omega$, pois $g_{i}$ é limite de uma sequência de comprimento $\omega$. Portanto, $g<x$. Desta forma, definindo $x^{\prime}=\left(x_{i}+1\right)_{i \in \mathbb{N}^{*}}$, temos que $x \in\left(g, x^{\prime}\right) \subset \bigcap_{n \in \omega} V_{n}$. Logo, $\bigcap_{n \in \omega} V_{n}$ é de fato aberto.

Teorema 4.1.7. O espaço $X$ do exemplo 4.1.5 é coletivamente normal. Em particular, $X$ é normal. 
Demonstração. Seja $\mathcal{H}=\left\{H_{j} \subset X: j \in J\right\}$ uma família discreta de fechados. Definimos $H=\bigcup_{j \in J} H_{j}$. Dado $C \subset X$, definimos uma função $s: \mathcal{P}(F) \longrightarrow F$ definida por $s(C)_{i}=\sup _{x \in C} x_{i}$ para cada $i \in \mathbb{N}^{*}$. Note que se $A \subset B$, temos $s(A) \leq s(B)$.

Para cada ordinal $\alpha<\omega_{1}$, vamos construir, por indução transfinita, uma cobertura $\mathfrak{J}_{\alpha}$ de $H$ por abertos disjuntos de $X$ com a seguinte propriedade: dados $\beta<\alpha<\omega$, podemos escolher um $U_{\beta} \in \mathfrak{J}_{\beta}$ para cada $U_{\alpha} \in \mathfrak{J}_{\alpha}$ de forma que:

1. $U_{\alpha} \subset U_{\beta}$

2. $\left|\left\{j \in J: U_{\alpha} \cap H_{j} \neq \emptyset\right\}\right| \geq 2 \Longrightarrow s\left(U_{\alpha}\right) \neq S\left(U_{\beta}\right)$

3. $\left|\left\{j \in J: U_{\beta} \cap H_{j} \neq \emptyset\right\}\right|<2 \Longrightarrow U_{\alpha}=U_{\beta}$

Primeiramente, definimos $\mathfrak{J}_{0}=\{X\}$. Em seguida, fixamos $\alpha<\omega_{1}$ e supomos $\mathfrak{J}_{\beta}$ construído para todo $\beta<\alpha$. Vamos então construir $\mathfrak{J}_{\alpha}$.

Começamos tratando do caso em que $\alpha$ é ordinal sucessor. Neste caso, fixemos $\beta$ tal que $\alpha=\beta+1$. Fixando $U \in \mathfrak{J}_{\beta}$, vamos construir uma família $P_{U} \subset \mathcal{P}(U)$ formada por abertos dois a dois disjuntos cobrindo $U \cap H$ de maneira que $\mathfrak{J}_{\alpha}=\bigcup_{U \in \mathfrak{J}_{\beta}} P_{U}$ satisfaça as condições 2 e 3 acima.

Se $U$ intersecciona no máximo 1 elemento de $\mathcal{H}$, temos que $P_{U}=\{U\}$ satisfaz as condições que queremos. Portanto, suponhamos agora que $U$ intersecciona 2 ou mais elementos de $\mathcal{H}$. Vamos dividir tal caso em duas situações.

Primeiramente, suponhamos que $\exists j \in \mathbb{N}^{*}, \operatorname{cf}\left(s(U)_{j}\right)=\omega$. Notemos que, neste caso, não podemos ter $\operatorname{cf}\left(s(U)_{j}\right)<\omega$, pois $\operatorname{cf}\left(f_{j}\right) \geq \omega_{1}$ para todo $f \in U \subset X$.

Seja $\left(\lambda_{n}\right)_{n \in \omega}$ uma sequência crescente cofinal em $s(U)_{j}$. A partir de tal sequência, construímos uma sequência de abertos $\left(V_{n}\right)_{n \in \omega}$ dada por $V_{0}=$ $\left\{x \in U: x_{j}<\lambda_{0}+1\right\}$ e por $V_{n}=\left\{x \in U: \lambda_{n-1}<x_{j}<\lambda_{n}+1\right\}$ para $n>$ 0 . Note que cada $V_{n}$ é de fato aberto, pois $V_{n}=U \cap \prod_{i \in \mathbb{N}^{*}} A_{i}$, onde $A_{j}=$ $\left(\lambda_{n-1}, \lambda_{n}+1\right)\left(\right.$ ou $\left.A_{j}=\right]-\infty, \lambda_{0}+1[$ no caso $n=0)$ e $A_{i}=F_{i}=\omega_{i}+1$ para $i \neq j$. Note também que tais abertos são disjuntos e cobrem $U$. Além disso, 
do modo como foram construídos, para cada $n \in \mathbb{N}^{*}$ temos $s\left(V_{n}\right)_{i}<s(U)_{i}$. Desta forma, $P_{U}=\left\{V_{n}: n \in \omega\right\}$ satisfaz as condições que queremos.

Por fim, suponhamos a situação complementar, ou seja, suponhamos que $\forall n \in \mathbb{N}^{*}, \operatorname{cf}\left(s(U)_{n}\right)>\omega$. Vamos dividir o aberto $U$ em $U=\bigcup_{n \in \mathbb{N}^{*}} U_{n}$, definindo para cada $n \in \mathbb{N}^{*}$ :

$$
U_{n}=\left\{x \in U: \operatorname{cf}\left(x_{i}\right) \leq \omega_{n}\right\}
$$

Vamos supor que para cada $n \in \mathbb{N}^{*}$, exista $g_{n} \in F$ com a seguinte propriedade:

$g_{n}<s(U)$ e o conjunto $\left\{x \in U_{n}: g_{n}<x\right\}$ intersecciona no máximo 1 elemento de $\mathcal{H}$.

Definimos então $f \in F$ dado por $f_{i}=\sup _{n \in \mathbb{N}^{*}}\left(g_{n}\right)_{i}$. Como estamos trabalhando com a situação em que $\forall i \in \mathbb{N}^{*}, \operatorname{cf}\left(s(U)_{i}\right)>\omega$, temos que $f<$ $s(U)$, pois toda coordenada de $f$ é limite de uma sequência enumerável.

Com $f$ assim construído, temos que o conjunto $\{x \in U: f<x\}$ intersecciona no máximo 1 elemento de $\mathcal{H}$. De fato, sejam $h, k \in H \cap\{x \in U: f<x\}$. Note que $\left(U_{n}\right)_{n \in \mathbb{N}^{*}}$ é uma sequência crescente temos que $h, k \in U_{m}$ para algum $m \in \mathbb{N}^{*}$ suficientemente grande. Além disso, temos que $g_{m}<h, k$. Logo, $h, k \in\left\{x \in U_{m}: g_{m}<x\right\}$. De tal forma, pela propriedade de $g_{m}$, temos que $h$ e $k$ pertencem ao mesmo elemento de $\mathcal{H}$.

A partir do $f \in F$ construído no parágrafo anterior, para cada $N \subset \mathbb{N}^{*}$ definimos:

$$
V_{N}=\left\{x \in U: \forall n \in N, x_{n} \leq f_{n} \text { e } \forall n \in \mathbb{N}^{*} \backslash N, x_{n}>f_{n}\right\}
$$

Definimos assim $P_{U}=\left\{V_{N}: N \subset \mathbb{N}^{*}\right\}$. Vamos mostrar que tal escolha de $P_{U}$ satisfaz as condições desejadas. Se para algum $N \subset \mathbb{N}^{*}$ tivermos $s\left(V_{N}\right)=s(U)$, então $N=\emptyset$ e portanto, pela construção de $f$, temos que $V_{\emptyset}=\{x \in U: f<x\}$, o qual intersecciona no máximo 1 elemento de $\mathcal{H}$. Além disso, $V_{N}$ é aberto pois podemos escrever $V_{N}=U \cap \prod_{i \in \mathbb{N}^{*}} N_{i}$, onde $\left.N_{i}=\right]-\inf , f_{i}+1\left[\right.$ para $i \in N$ e $\left.N_{i}=\right] f_{i}, \inf \left[\operatorname{para} i \in \mathbb{N}^{*} \backslash N\right.$. 
Vamos agora demonstrar que existem $g_{n} \in F$ com tal propriedade. Fixemos $n \in \mathbb{N}^{*}$. Vamos supor que tal $g_{n}$ não exista, isto é, suponhamos que:

[*] Para todo $f<s(U)$ existem $h, k \in U_{n}$ tais que $f<h, k$ e $h$ e $k$ pertencem a elementos distintos de $\mathcal{H}$.

Vamos particionar $\mathbb{N}^{*}$ da seguinte maneira: para $1<i \leq n$ definimos $M_{i}=\left\{j \in \mathbb{N}^{*}: \operatorname{cf}\left(s(U)_{j}\right)=\omega_{i}\right\}$ e também $M=\left\{j \in \mathbb{N}^{*}: \operatorname{cf}\left(s(U)_{j}\right)>\omega_{n}\right\}$. A hipótese de que $\forall n \in \mathbb{N}^{*}, \operatorname{cf}\left(s(U)_{n}\right)>\omega$ garante que $\mathbb{N}^{*}=\bigcup_{1<i \leq n} M_{i} \cup M$, sendo essa uma união disjunta. Com tal partição, para cada $i \leq n$ e para cada $j \in M_{i}$, podemos escolher um conjunto $\left\{p_{j, \sigma}: \sigma<\omega_{i}\right\} \subset s(U)_{j}$ cofinal $\operatorname{com}\left(p_{j, \sigma}\right)_{\sigma<\omega_{i}}$ crescente.

Considere também $R=\prod_{i \leq n} \omega_{i}$. Temos que $|R|=\omega_{n}$. Portanto, existe uma bijeção $f: \omega_{n} \longrightarrow \omega_{n} \times R$. Fixemos então a sequência $\left(r_{\lambda}\right)_{\lambda \in \omega_{n}}$ onde $r_{\lambda}$ é a segunda coordenada de $f(\lambda)$. Note que tal sequência possui a seguinte propriedade: fixados $r \in R$ e $\delta \in \omega_{n}$, existe $\delta<\gamma<\omega_{n}$ tal que $r_{\gamma}=r$. De fato, escolha $\alpha \in \omega_{n}$ tal que para todo $\beta \leq \delta$ não tenhamos $\alpha$ como a primeira coordenada de $f(\beta)$. Tal $\alpha$ existe pois, caso contrário, como $f$ é bijetora, teríamos que a restrição à $\delta$ da projeção na primeira coordenada de $f$ seria uma bijeção entre $\delta$ e $\omega_{n}$. Escolhemos então $\gamma \in \omega$ tal que $f(\gamma)=(\alpha, r)$. Tal $\gamma$ existe pois $f$ é bijetora. Além disso, $\gamma>\delta$ pela construção de $\alpha$. Por definição, $r_{\gamma}=r$, como queríamos.

Após escolher tais sequências, vamos construir indutivamente um trio $\left(f_{\lambda}, h_{\lambda}, k_{\lambda}\right)$ para cada $\lambda \in \omega_{n}, \operatorname{com} f_{\lambda} \in F$ e $h_{\lambda}, k_{\lambda} \in U_{n}$.

Primeiramente, definimos $f_{0}$ dado por $\left(f_{0}\right)_{j}=p_{j,\left(r_{0}\right)_{i}}$ se $j \in M_{i}(i \leq n) \mathrm{e}$ $\left(f_{0}\right)_{j}=0$ se $j \in M$. Usamos então a hipótese [*] para escolher $h_{0}, k_{0} \in U_{n}$ pertencentes a elementos distintos de $\mathcal{H}$ e tais que $f_{0}<h_{0}, k_{0}$.

Então, seja $\lambda \in \omega_{n}$ tal que $h_{\gamma}$ e $k_{\gamma}$ já foram construídos para cada $\gamma<\lambda$. Definimos $\left(f_{\lambda}\right)_{j}=p_{j,\left(r_{\lambda}\right)_{i}}$ para $j \in M_{i}(i \leq n)$ e $\left(f_{\lambda}\right)_{j}=$ $\sup \left\{h_{j}: h \in \bigcup_{\gamma<\lambda}\left\{h_{\gamma}, k_{\gamma}\right\}\right\}$ para $j \in M$. Note que $f_{\lambda}$ foi construído de maneira que $f_{\lambda}<s(U)$. Portanto, novamente pela hipótese $[*]$, podemos escolher $h_{\lambda}, k_{\lambda} \in U_{n}$ pertencentes cada um a elementos distintos de $\mathcal{H}$ tais que $f_{\lambda}<h_{\lambda}, k_{\lambda}$.

Construída a sequência dos $f_{\lambda}, k_{\lambda}, h_{\lambda}$, definimos $g \in F$ dado por $g_{j}=$ 
$s(U)_{j}$ para $j \in \mathbb{N}^{*} \backslash M$ e $g_{j}=\sup _{\lambda<\omega_{n}}\left(h_{\lambda}\right)_{j}$ para $j \in M$. Se $j \in M$, temos $\operatorname{cf}\left(g_{j}\right) \leq \omega_{n}$. De fato, por construção, temos que $\left(\max \left\{\left(h_{\lambda}\right)_{j},\left(k_{\lambda}\right)_{j}\right\}\right)_{\lambda \in \omega_{n}}$ é uma sequência cofinal em $g_{j}$ de cumprimento $\omega_{n}$. Além disso, se $j \in \mathbb{N}^{*} \backslash M$, então $\operatorname{cf}\left(g_{j}\right)=\operatorname{cf}(s(U))>\omega$ por hipótese. Portanto $g \in X$. Além disso, como $\forall \lambda \in \omega_{n}, k_{\lambda} \in U_{n} \subset U$, temos $g \leq s(U)$.

Lembrando que a família $\mathcal{H}$ é discreta, existe uma caixa aberta $V=$ $\prod_{i \in \mathbb{N}^{*}} V_{i}$ tal que $g \in V$ e $V$ intersecciona no máximo 1 elemento de $\mathcal{H}$. Podemos supor sem perda de generalidade que, para cada $i \in \mathbb{N}^{*}, V_{i}$ é um intervalo aberto $] a_{i}, b_{i}\left[\operatorname{com} a_{i}, b_{i} \in \omega_{i}\right.$. Definimos assim $a=\left(a_{i}\right)_{i \in \mathbb{N}^{*}}$. Como $g \leq s(U)$, temos $a<s(U)$.

Para cada $i \leq n$ e $j \in M_{i}$, usamos o fato de que $\left\{p_{j, \sigma}: \sigma \in \omega_{i}\right\}$ é cofinal em $s(U)_{j}$ para encontrar $\omega_{j}$ tal que $a_{j}<p_{j, \sigma_{j}}$. Definimos $\mu_{i}=$ $\sup \sigma_{j}: j \in M_{i}$. Como $M_{i} \subset \mathbb{N}^{*}$ é enumerável e $\operatorname{cf}\left(\omega_{i}\right)=\omega_{i}>\omega$, temos $\mu_{i}<\omega_{i}$. Definimos então $r \in R$ dado por $r_{i}=\mu_{i}$ para cada $i \leq n$. Já para $j \in M$, como $a<g$, escolhemos $\sigma_{j}$ tal que $a_{j}<\left(h_{\sigma_{j}}\right)_{j}$. Definimos agora $\sigma=\sup \sigma_{j}: j \in M$.

Pela propriedade da sequência $\left(r_{\lambda}\right)_{\lambda \in \omega_{n}}$, podemos encontrar $\left.\gamma \in\right] \sigma, \omega_{n}[$ tal que $r=r_{\gamma}$. Assim, se $j \in M$, temos $a_{j}<\left(h_{\gamma}\right)_{j}<g_{j}$, portanto $\left(h_{\gamma}\right)_{j} \in V_{j}$. Por outro lado, se $j \in M_{i}(i \leq n)$, então

$$
a_{j}<p_{j, r_{i}}=p_{j,\left(r_{\gamma}\right)_{i}}<\left(h_{\gamma}\right)_{j} \leq s(U)_{j}=g_{j}
$$

e portanto $\left(h_{\gamma}\right)_{j} \in V_{j}$. Assim, temos que $h_{\gamma} \in V$, e da mesma maneira mostramos que $k_{\gamma} \in V$, o que é um absurdo, pois $V$ intersecciona no máximo 1 elemento de $\mathcal{H}$. Provamos assim a existência dos pontos $g_{n}$, concluindo a construção de $P_{U}$.

Construído então $P_{U}$, definimos $\mathfrak{J}_{\alpha}=\bigcup_{U \in \mathfrak{J}_{\beta}} P_{U}$

Para completar a construção de $\mathfrak{J}_{\alpha}$, falta apenas o caso em que $\alpha$ é ordinal limite. Neste caso, para cada $\beta<\alpha$ e cada $x \in H$ definimos $U_{x}(\beta)$ como o único elemento de $\mathfrak{J}_{\beta}$ ao qual $x$ pertence. Definimos então $U_{x}=\bigcap_{\beta<\alpha} U_{x}(\beta)$. Como $\alpha$ é enumerável, temos que $U_{x}$ é aberto pelo lema 4.1.6. O fato de $\mathfrak{J}_{\beta}$ ser disjunto para cada $\beta<\alpha$ garante que $\mathfrak{J}_{\alpha}$ também o seja. Além disso, dado $x \in H$, se $U_{x}$ intercepta 2 ou mais elementos de $\mathcal{H}$, então $U_{x}(\beta)$ e $U_{x}(\beta+1)$ 
também o fazem, para algum $\beta<\alpha$. Então temos $s\left(U_{x}(\alpha)\right) \leq s\left(U_{x}(\beta+1)\right)<$ $s\left(U_{x}(\beta)\right)$, portanto $\mathfrak{J}_{\alpha}$ satisfaz a condição 2 . Da mesma forma, se $U_{x}(\beta)$ para algum $\beta<\alpha$, então $U_{x}(\beta)=U_{x}(\gamma)$ para todo $\gamma$ tal que $\alpha<\beta<\gamma$, logo $U_{x}(\alpha)=U_{x}$, como queríamos.

Temos então construídas as famílias $\mathfrak{J}_{\alpha}$ para cada $\alpha<\omega_{1}$. Vamos mostrar, por fim, que a existência de tais famílias implicam que $X$ é coletivamente normal.

Fixemos $x \in H$. Para cada $\alpha \in \omega_{1}, \mathfrak{J}_{\alpha}$ cobre $H$ com abertos disjuntos. Logo, existe um único $U_{\alpha} \in \mathfrak{J}_{\alpha}$ tal que $x \in U_{\alpha}$. Pela propriedade (1) da construção de $\mathfrak{J}_{\alpha}$, temos que se $\alpha<\beta \omega_{1}$, então $U_{\beta}<U_{\alpha}$ e, portanto, $s\left(U_{\beta}\right) \leq s\left(U_{\alpha}\right)$. Pela propriedade $(2)$ da definição de $\mathfrak{J}_{\alpha}$, temos que se $U_{\beta}$ intersecciona mais do que 1 elemento de $\mathfrak{H}$, então $s\left(U_{\beta}\right)<s\left(U_{\alpha}\right)$. Neste caso, existe algum $i \in \omega$ tal que $s\left(U_{\beta}\right)_{i}<s\left(U_{\alpha}\right)_{i}$. Fixado tal $n$, note que podemos "diminuir" $s\left(U_{\alpha}\right)$ somente um número finito de vezes, pois toda sequência decrescente de ordinais é eventualmente constante, como vimos na demonstração do teorema 3.4.1. Sendo assim, podemos encontrar um $\alpha_{x} \in \omega_{1}$ suficientemente grande tal que $U_{\alpha_{x}}$ intersecciona no máximo 1 único elemento de $\mathfrak{H}$. Pela propriedade (3), temos que $U_{\beta}=U_{\alpha_{x}}$ para todo $\alpha_{x} \leq \beta<\omega_{1}$.

Definimos então $U_{j}=\bigcup_{x \in H_{j}} U_{\alpha_{x}}$ para cada $j \in J$. Vamos mostrar que a família de abertos $\left\{U_{j}: j \in J\right\}$ separa os elementos de $\mathfrak{H}$ como na definição 1.2.11: Sejam $x, y$ pertencentes a elementos distintos de $\mathfrak{H}$. Seja $\alpha \in \omega_{1}$ tal que $\alpha>\max \left\{\alpha_{x}, \alpha_{y}\right\}$, com $\alpha_{x}, \alpha_{y}$ como definidos no parágrafo anterior (de tal modo que $x \in U_{\alpha_{x}}$ e $y \in U_{\alpha_{y}}$ ). Temos então que $U_{\alpha_{x}}, U_{\alpha_{y}} \in \mathfrak{J}_{\alpha}$, logo são disjuntos. Além disso, pela construção, temos que $U_{\alpha_{x}}$ e $U_{\alpha_{y}}$ só interseccionam cada um somente 1 único elemento de $\mathfrak{H}$, como queríamos.

Teorema 4.1.8. Seja $X$ o espaço construído no exemplo 4.1.5. Temos que $X \times \mathbb{I}$ não é normal.

Demonstração. Vamos mostrar que $X$ satisfaz a condição 4.1.3 do teorema 4.1.3. Para cada $n \in \mathbb{N}^{*}$, defino $F_{n}=\left\{x \in X: \forall i \leq n, x_{i}=\omega_{i}\right\}$. Vamos mostrar que $\bigcap_{n \in \mathbb{N}^{*}} F_{n}=\emptyset$. Suponha $p \in \bigcap_{n \in \mathbb{N}^{*}} F_{n}$. Como $p \in X$, existe $k \in \mathbb{N}^{*}$ tal que para todo $i \in \mathbb{N}^{*}$ temos $\omega_{1} \leq \operatorname{cf}\left(p_{i}\right)<\omega_{k}$. No entanto, como $p \in F_{k}$ temos que $p_{k}=\omega_{k}$, o que contradiz $p \in X$, pois $\omega_{k}$ é regular. Portanto, 
temos de fato $\bigcap_{n \in \mathbb{N}^{*}} F_{n}=\emptyset$.

Vamos mostrar agora que que $F_{n}$ é fechado para todo $n \in \mathbb{N}^{*}$. Dado $y \in A_{n}{ }^{c}$, temos que existe $j \leq n$ tal que $y_{j} \neq \omega_{j}$. Assim podemos considerar a caixa aberta $V=\prod_{i \in I}\left(V_{i}\right)$ dada por $V_{j}=\left[0, \omega_{j}\right)$ e $V_{i}=\omega_{i}$ para $i \neq j$. Temos então que $y \in X \cap V \subset A_{n}{ }^{c}$.

Seja $\left(A_{n}\right)_{n \in \mathbb{N}^{*}}$ uma sequência de abertos tal que $F_{n} \subset A_{n} \subset X$ para todo $n \in \mathbb{N}^{*}$. Vamos mostrar que $\bigcap_{n \in \mathbb{N}^{*}} A_{n} \neq \emptyset$ e, portanto, pelo teorema 4.1.3, $X \times \mathbb{I}$ não pode ser normal.

Suponhamos que existam para cada $n \in \mathbb{N}^{*}$ um ponto $f_{n} \in X$ tal que $\left\{h \in X: f_{n}<h\right\} \subset U_{n}$ e $\left(f_{n}\right)_{i}<\omega_{i}$ para cada $i \in \mathbb{N}^{*}$. A partir de tais pontos, vamos construir $g \in \bigcap_{n \in \mathbb{N}^{*}} A_{n}$. Fixamos $g_{1}=\omega_{1}$. Para cada $i>1$ encontramos um ordinal $g_{i}$ tal que $\left(f_{n}\right)_{i}<g_{i}<\omega_{i}$ para todo $n>1 \mathrm{e}$ também tenha cofinalidade $\operatorname{cf}\left(g_{i}\right)=\omega_{1}$. De fato existe um ordinal com tais propriedades, pois construir uma sequência crescente de comprimento $\omega_{i}$ de ordinais arbitrariamente grandes mesmo limitada por $\omega_{i}$, com $i>1$. Temos então que $g=\left(g_{i}\right)_{i \in \mathbb{N}^{*}} \in X$. Para cada $n \in \mathbb{N}^{*}$, como $f_{n}<g$, temos que $g \in U_{n}$, como queríamos.

Agora vamos provar que tais pontos $f_{n}$ existem. Para facilitar a notação, definimos $C_{j}=x \in F_{j}: \forall i>j, x_{i}<\omega_{i}$ para cada $j \in \mathbb{N}^{*}$. Seja $k_{n} \in C_{n}$ qualquer. Note que como $F_{n} \subset A_{n}$ e $A_{n}$ é aberto, temos que $\left\{x \in C_{n}: k_{n}<x\right\} \subset$ $A_{n}$. A partir de $k_{n}$, vamos construir recursivamente uma família de pontos $\left\{k_{j} \in C_{j}: 1 \leq j \leq n\right\}$ tais que $\left\{x \in C_{j}: k_{j}<x\right\} \subset A_{n}$. De tal forma, obteremos o ponto $f_{n}$ que desejamos por $f_{n}=k_{1}$.

Vamos construir $k_{j}$ supondo $k_{j+1}$ já construído. Definimos $h_{0} \in X$ dado por $\left(h_{0}\right)_{j}=0$ e $\left(h_{0}\right)_{i}=\left(k_{j}\right)_{i}$ para cada $i \neq j$. Usando o lema de Zorn, podemos construir uma sequência bem ordenada maximal $\left(h_{\alpha}\right)_{\alpha<\lambda}$, para algum ordinal $\lambda$, tal que para cada $\alpha<\lambda$ temos $h<h_{\alpha}$ e $h_{\alpha} \in C_{j} \backslash A_{n}$. Se $\lambda=0$, não há mais o que demonstrar, logo trabalharemos com $\lambda>0$. Definimos $h^{\prime} \in X$ dado por $h_{i}^{\prime}=\sup _{\alpha<\lambda}\left(h_{\alpha}\right)_{i}$ para cada $i \in \mathbb{N}^{*}$.

Como a sequência é maximal, temos que $\lambda \leq \omega_{j}$. Vamos mostrar que $\lambda<\omega_{j}$. Suponhamos que $\lambda=\omega_{j}$. Neste caso, teríamos $h^{\prime} \in C_{j+1}$ e $k_{j+1}<h^{\prime}$ e portanto, como supomos $k_{j+1}$ construída, $h^{\prime} \in A_{n}$. Como $A_{n}$ é aberto, existe um ponto $h^{*} \in F, h^{*}<h^{\prime}$, tal que o intervalo $] h^{*}, h^{\prime}\left[\subset A_{n}\right.$ (veja a 
observação 4.1.4). Porém, como $h^{*}<h^{\prime}$, para cada $i>j$ podemos encontrar um ordinal $\alpha_{i}<\lambda$ tal que $h^{*}<\left(h_{\alpha_{i}}\right)_{i}$. Definindo $\alpha=\sup _{i>n} \alpha_{i}$, temos que $h^{*}<h_{\alpha}<h^{\prime}$. Logo $h_{\alpha} \in A_{n}$, o que contradiz $k_{\alpha} \in C_{j} \backslash A_{n}$. Portanto de fato temos $\lambda<\omega_{j}$.

Por fim, definimos $f_{j}$ por $\left(f_{j}\right)_{i}=h_{i}^{\prime}+\omega_{1}$ para $i>j$ e $\left(f_{j}\right)_{i}=\omega_{i}$ para $i \leq j$. Temos então $f_{j} \in C_{j}$ e a maximalidade da sequência $\left(h_{\alpha}\right)_{\alpha<\lambda}$ garante que $\left\{x \in C_{j}: f_{j}<x\right\} \subset U_{n}$, o que completa a demonstração.

Uma desvantagem de se trabalhar com produtos é que os espaços com eles construídos podem ter cardinalidades altas. Podemos, por exemplo, verificar que o espaço $X$ do exemplo 4.1 .5 tem cardinalidade $\aleph_{\omega}{ }^{\aleph_{0}}$. Pode-se, no entanto, a partir de tal exemplo construir espaços de Dowker de menor cardinalidade. Shelah e Kojman em [KS98] aplicaram a teoria pcf para construir um subespaço de $X$ de cardinalidade $\aleph_{\omega+1}$, o qual também é de Dowker. Este resultado também é abordado em [Fer11]

\subsection{Um produto não normal de espaços $\kappa$-metrizáveis}

Em [Nyi75], Peter Nyikos levantou a seguinte questão: o produto de um espaço metrizável por um espaço $\kappa$-metrizável (definição 1.2.30), para algum $\kappa$ infinito qualquer, é normal? Como a definição de espaço $\kappa$-metrizável é local, temos que tais espaços são não apenas normais como hereditariamente normais. A demonstração deste fato é basicamente a mesma de que todo espaço metrizável é normal.

J. E. Vaughan mostrou em [Vau75] que a resposta à pergunta de Nyikos é negativa, através do seguinte exemplo.

Exemplo 4.2.1. Seja $D_{1}$ o espaço topológico formado por $\omega_{1}$ equipado com a topologia discreta, e definimos $\mathfrak{D}_{1}=D_{1}^{\omega}$ como produto de Tychonoff. Seja $D_{1}^{*}=\omega_{1}+1$ equipado com a topologia gerada pela seguinte base $\mathcal{B}=$ $\left.\left.\left\{\{\alpha\}: \alpha<\omega_{1}\right\} \cup\{] \beta, \omega_{1}\right]: \beta<\omega_{1}\right\}$. Definimos então $B_{1}=\square^{\omega} D_{1}^{*}$. Temos então que $\mathfrak{D}_{1}$ é metrizável, $B_{1}$ é $\omega_{1}$-metrizável, porém $\mathfrak{D}_{1} \times B_{1}$ não é normal. 
Além de responder a pergunta de Nyikos, o exemplo 4.2.1 também serve de contra exemplo para uma conjectura, também de Vaughan. Em [Vau72], é introduzido o conceito de espaço linearmente estratificável, além da conjectura de que o produto de dois espaços com tal propriedade seria paracompacto. Pela definição, pode-se ver que todo espaço $\omega_{\mu}$-metrizável é em particular $\omega_{\mu}$-estratificável. Portanto o espaço $B_{1}$ é $\omega_{1}$-estratificável. Além disso, sabe-se que todo espaço metrizável é estratificável, portanto $\mathfrak{D}_{1}$ é estratificável. Como mostraremos que $\mathfrak{D}_{1} \times B_{1}$ não é normal, tal produto em particular não é paracompacto, negando a conjectura.

Demonstração do exemplo 4.2.1. Primeiramente, como $D_{1}$ é discreto, admite a métrica discreta. Logo $\mathfrak{D}_{1}$ é metrizável, por ser um produto enumerável de espaços metrizáveis. Além disso, temos que $B_{1}$ é $\omega_{1}$-metrizável, e construiremos as bases locais que o caracterizam como tal.

Para facilitar as próximas construções, vamos definir bases locais para $\mathfrak{D}_{1}$ e $B_{1}$. Dado um ponto $x=\left(x_{i}\right)_{i \in \omega} \in \mathfrak{D}_{1}$ e um natural $n \in \omega$, definimos $n(x)=\left\{y \in \mathfrak{D}_{1}: \forall i \leq n, y_{i}=x_{i}\right\}$. Assim, $\{n(x): n \in \omega\}$ é uma base local para $x$. Considere agora $q \in B_{1}$ e um ordinal $\alpha \in \omega_{1}$. Definimos então $\alpha(q)=\prod_{i \in \omega} Q_{i}$, onde $Q_{i}=\left\{q_{i}\right\}$ sempre que $q_{i}<\omega_{1}$, e $\left.\left.Q_{i}=\right] \alpha, \omega_{1}\right]$ para todo $i \in \omega$ tal que $q_{i}=\omega_{1}$. Da mesma maneira, $\left\{\alpha(q): \alpha<\omega_{1}\right\}$ é uma base local para $q$, a qual também testemunha a $\omega_{1}$-metrizabilidade de $B_{1}$. Note que para que tal família seja base local de $q$ precisamos do fato de que $\operatorname{cf}\left(\omega_{1}\right) \neq \omega$. De fato, se existisse uma sequência $\left(\gamma_{n}\right)_{n \in \omega}$ cofinal em $\omega_{1}$, então $\left.\left.\prod_{n \in \omega}\right] \gamma_{n}, \omega_{1}\right]$ seria uma vizinhança aberta do ponto $q_{\omega_{1}}=\left(\omega_{1}\right)_{n \in \omega}$ a qual não contém nenhuma vizinhança do tipo $\alpha\left(q_{\omega_{1}}\right)$.

Vamos mostrar que $\mathfrak{D}_{1} \times B_{1}$ não é normal. Para isso, notemos que, como conjuntos, temos $\mathfrak{D}_{1} \subset B_{1}$, apesar de a topologia de $\mathfrak{D}_{1}$ não coincidir com a topologia de subespaço de $B_{1}$. Definimos então:

$$
H=\left\{(x, x) \in \mathfrak{D}_{1} \times B_{1}: x \in \mathfrak{D}_{1}\right\} \quad \text { e } \quad K=\mathfrak{D}_{1} \times\left(B_{1} \backslash \mathfrak{D}_{1}\right)
$$

Vamos mostrar $H$ e $K$ são fechados disjuntos que não podem ser separados por abertos disjuntos. Primeiramente, $K$ é claramente fechado. De fato, o complementar de $K$ é o quadrado $\mathfrak{D}_{1} \times \mathfrak{D}_{1}$. Como $H \subset \mathfrak{D}_{1} \times \mathfrak{D}_{1}$, temos 
$H \cap K=\emptyset$. Também é fácil de ver que $H$ é fechado: seja $(x, y) \notin H$. Se $y \in \mathfrak{D}_{1}$, então existe algum $k \in \omega$ tal que $x_{k} \neq y_{k}$. Neste caso, temos $k(x) \times\{y\}$ é uma vizinhança de $(x, y)$ que não intersecciona $H$. No caso em que $y \notin \mathfrak{D}_{1}$, existe algum $n \in \omega$ tal que $y_{n}=\omega_{1}$. Se escolhermos algum $\alpha$ tal que $x_{n}<\alpha<\omega_{1}$, temos que $n(x) \times \alpha(y)$ também é uma vizinhança de $(x, y)$ que não intercepta $H$, como queríamos.

Agora, seja $V$ um aberto qualquer tal que $K \subset V$. Vamos construir um ponto $(x, x)$ em $H$ cujas vizinhanças sempre interseccionam $V$. Construire$\operatorname{mos} x=\left(x_{i}\right)_{i \in \omega}$ coordenada a coordenada, por indução.

Começamos com os pontos $p_{0} \in D_{1}$ e $q_{0} \in B_{1} \backslash \mathfrak{D}_{1}$ dados por $p_{0}=(0,0, \ldots)$ e $q_{0}=\left(\omega_{1}, \omega_{1}, \ldots\right)$. Como $\left(p_{0}, q_{0}\right) \in K \subset V$, podemos achar $\left(m_{0}, q_{0}\right) \in \omega \times \omega_{1}$ tal que $m_{0}\left(p_{0}\right) \times \alpha_{0}\left(q_{0}\right) \subset V$, já que $V$ é aberto. Escolhemos então $x_{0}$ tal que $\alpha_{0}<x_{0}<\omega_{1}$.

Agora fixemos $k \in \omega$ e suponhamos que $x_{i}$ foi construído para todo $i \leq k$. Definimos $p_{k+1}=\left(x_{0}, x_{1}, \ldots, x_{k}, 0,0, \ldots\right)$ e $q_{k+1}=\left(x_{0}, x_{1}, \ldots, x_{k}, \omega_{1}, \omega_{1}, \ldots\right)$. Temos que $\left(p_{k+1}, q_{k+1}\right) \in K \subset V$. Da mesma maneira, podemos escolher $\left(m_{k+1}, \alpha_{k+1}\right) \in \omega \times \omega_{1}$ de maneira que $m_{k+1}\left(p_{k+1}\right) \times \alpha_{k+1}\left(q_{k+1}\right) \subset V$. Escolhemos então $x_{k+1}$ tal que $\max \left\{\alpha_{k+1}, x_{k}\right\}<x_{k+1}<\omega_{1}$. Concluímos assim a construção de $x$.

Como a cada passo limitamos $x_{n}<\omega_{1}$, temos que de fato $(x, x) \in H$. Seja $k \in \omega$. Vamos mostrar que o ponto $\left(p_{k+1}, x\right)$ pertence à intersecção $(k(x) \times\{x\}) \cap\left(n_{k+1}\left(p_{k+1}\right) \times \alpha_{k+1}\left(q_{k+1}\right)\right), \log$ qualquer vizinhança do ponto $(x, x)$ intersecciona $V$ e, portanto, qualquer aberto $U \supset H$ intersecciona $V$, concluindo o resultado.

Lembrando que $p_{k+1}=\left(x_{0}, x_{1}, \ldots, x_{k}, 0,0, \ldots\right)$, temos $p_{k+1} \in k(x)$. Além disso, construímos $x$ de maneira que a sequência $\left(x_{n}\right)_{n \in \omega}$ fosse crescente, logo temos que para cada $n>k, \alpha_{k+1}<x_{n}$, o que garante que $x \in \alpha_{k+1}\left(q_{k+1}\right)$.

Corolário 4.2.2. Seja $B_{1}$ o espaço construído no exemplo 4.2.1. Entãa para qualquer $n \in \omega$ temos que $B_{1}{ }^{n}$ é homeomorfo a $B_{1}$, porém o produto de Tychonoff $B_{1}{ }^{\omega}$ não é normal.

Este corolário responde uma questão por E. Michael em [Mic71]. Em tal artigo, Michael deu um exemplo de um espaço $Y$ tal que para todo $n \in \omega, Y^{n}$ 
é normal, porém $Y^{\omega}$ não o é. Tal exemplo levou à pergunta sobre a existência de um exemplo de espaço com a mesma propriedade, apenas substituindo "normal" por "hereditariamente normal" . Em [Mic71], Vaughan demonstra que todo espaço $\omega_{1}$-estratificável é hereditariamente paracompacto, e portanto, hereditariamente normal (resultado originalmente demonstrado para espaços $\kappa$-metrizáveis por Juhász em [Juh65]). Portanto, o corolário 4.2.2 mostra que o espaço $B_{1}$ é um exemplo de solução para a pergunta de Michael.

Demonstração. Primeiramente, notemos que na aritmética cardinal temos $\aleph_{0} \times n=\aleph_{0}$ para qualquer $n$ finito. Logo, temos uma bijeção natural entre $B_{1}=\square^{\omega} D_{1}^{*}$ e $B_{1}^{n}$. Além disso, como tal bijeção constitui apenas uma reordenação de índices, temos que tal bijeção é um homeomorfismo.

Vamos mostrar agora que $B_{1}^{\omega}$ não é normal. Primeiramente, note que $\omega$ é um subconjunto discreto fechado de $\mathfrak{D}_{1}^{*}$. Portanto, temos um subespaço fechado discreto de cardinalidade $2^{\aleph_{0}}$ em $B$. Dentro deste, podemos encontrar um subconjunto, também fechado e discreto, de cardinalidade $\aleph_{1}$. Em outras palavras, podemos encontrar uma cópia homeomorfa de $D_{1}$ em $B_{1}$, a qual é fechada. Desta forma, obtemos uma cópia homeomorfa de $\mathfrak{D}_{1}$ em $B_{1}^{\omega}$, fechada. Semelhantemente ao parágrafro anterior, temos que $B_{1}^{\omega}$ é homeomorfo a $B_{1}^{\omega} \times B_{1}$. A partir de tal fato, encontramos uma cópia homeomorfa de $\mathfrak{D}_{1} \times B_{1}$ fechada em $B_{1}^{\omega} \times B_{1}$. Como já mostramos que $\mathfrak{D}_{1} \times B_{1}$ não é normal, concluímos que $B_{1}^{\omega}$ também não o é.

Note que na demonstração do exemplo 4.2.1, para construir a sequência $\left(x_{i}\right)_{i \in \omega}$ precisamos do fato de que $\operatorname{cf}\left(\omega_{1}\right) \neq \omega$, porém o resultado pode ser analogamente estendido para qualquer cardinalidade, como mostra o teorema 4.2.3. Para tal fim, generalizamos a construção de $\mathfrak{D}_{1}$ e $B_{1}$ a seguir:

Seja $\omega_{\mu}$ o $\mu$-ésimo cardinal. Definimos então $D_{\mu}$ por $\omega_{\mu}$ equipado com a topologia discreta. Chamaremos de $\mathfrak{D}_{\mu}$ o produto de Tychonoff $D_{\mu}{ }^{\omega}$. Definimos também $D_{\mu}^{*}=\omega_{\mu}+1$ equipado com a topologia gerada pela base $\mathcal{B}=\left\{\{\alpha\}: \alpha<\omega_{\mu}\right\} \cup\left\{\left(\beta, \omega_{\mu}\right]: \beta<\omega_{\mu}\right\}$, e assim definimos $B_{\mu}=\square^{\omega} D_{\mu}^{*}$.

Teorema 4.2.3. O espaço $\mathfrak{D}_{\mu} \times B_{\mu}$ não é normal. 
Demonstração. Nos casos em que $\operatorname{cf}\left(\omega_{\mu}\right)>\omega$, a demonstração é exatamente a mesma que do exemplo 4.2.1. Nos demais casos, a demonstração é análoga ao caso $\mu=0$, o qual foi demonstrado por Van Dowen em [vD75].

Vamos então mostrar que $\mathfrak{D}_{0} \times B_{0}$ não é normal. Como $\operatorname{cf}(\omega)=\omega$, não podemos usar para os pontos de $B_{0}$ as mesmas bases locais do exemplo 4.2.1, então construiremos outras da seguinte maneira. Para cada ponto $y \in B_{0}$, $\operatorname{dados} f: \omega \longrightarrow \omega$ e $i \in \omega$, definimos $U(y, f, i)=\prod_{j \leq n}\left\{y_{j}\right\} \times \prod_{j>n}(f(j), \omega]$. Assim, temos que $\left\{U(y, f, n): f \in \omega^{\omega}\right\}$ é uma base local para qualquer $y$ tal que $y_{i}<\omega$ para todo $i \leq n$.

Como ferramenta para este resultado, vamos mostrar que $\mathfrak{D}_{0}$, considerado como subconjunto de $B_{0}$, não é $F_{\sigma}$. Para cada $n \in \omega$, seja $F_{n} \subset \mathfrak{D}_{0}$ um conjunto fechado em $B_{0}$. Por indução nas coordenadas, vamos construir um ponto $x=\left(x_{n}\right)_{n \in \omega} \in D_{0}$ tal que $x \notin \bigcup_{n \in \omega} F_{n}$, o que conclui que $D_{0}$ não é $F_{\sigma}$.

Começamos com $x_{0}=0$. Fixamos então $n \in \omega$ e supomos $x_{i}$ construído para cada $i \leq n$ de maneira de que para cada $i<n$ exista uma função $f_{i}: \omega \longrightarrow \omega$ tal que $U\left(x_{i}, f_{i}, i\right) \cap F_{i}=\emptyset$. Note que o ponto $y_{n}=\left(x_{0}, x_{1}, \ldots, x_{n}, \omega, \omega, \ldots\right)$ não pertence $\mathfrak{D}_{0}$. Logo $y_{n} \notin F_{n}$, de onde podemos escolher uma função $f_{n}: \omega \longrightarrow \omega$ tal que $U\left(y_{n}, f_{n}, n\right) \cap F_{n}=\emptyset$. Escolhemos então $x_{n+1} \in \omega$ tal que $x_{n+1}>f_{i}(n+1)$ para todo $i \leq n$, o que completa a indução.

Sejam $H, K \subset \mathfrak{D}_{0} \times B_{0}$ fechados disjuntos análogos aos construídos no exemplo 4.2.1. Suponhamos $U, V$ abertos em $\mathfrak{D}_{0} \times B_{0}$ tais que $H \subset U$ e $K \subset V$. Para o próximo passo, vamos construir uma métrica $d$ sobre $B_{0}$. Note que essa métrica não pode ser compatível com $B_{0}$, pois se fosse, teríamos $\mathfrak{D}_{0} \times B_{0}$ metrizável, logo normal. Tal métrica seria, na verdade, compatível com o espaço $\left(D_{0}^{*}\right)^{\omega}$ com o produto de Tychonoff, e portanto sua restrição a $\mathfrak{D}_{0}$ é compatível com o mesmo. Para cada $D_{0}^{*}$, definimos a distância $d_{i}: D_{0}^{*} \times D_{0}^{*} \longrightarrow \mathbb{R}_{+}$dada por $d_{i}(x, y)=\left|\frac{1}{x}-\frac{1}{y}\right|$ para cada $x, y \in \omega$, onde definimos $\frac{1}{\omega}=0$. Definimos então a métrica $d: B_{0} \times B_{0} \longrightarrow \mathbb{R}_{+}$dada por $d(x, y)=\sum_{i \in \omega} 2^{-i} d_{i}\left(x_{i}, y_{i}\right)$. 
Usaremos $d$ para dividir $\mathfrak{D}_{0}$ em $\omega$ partes. Para cada $n \in \omega$ definimos

$$
P_{n}=\left\{x \in \mathfrak{D}_{0}: S\left(x, \frac{1}{n}\right) \times\{x\} \subset U\right\}
$$

onde $S(x, \epsilon)=\left\{y \in \mathfrak{D}_{0}: d(x, y)<\epsilon\right\}$ é a bola aberta da métrica $d$ em $\mathfrak{D}_{0}$. Temos que $\mathfrak{D}_{0}=\bigcup_{n \in \omega} P_{n}$. Como demonstramos que $\mathfrak{D}_{0}$ não pode ser $F_{\sigma}$, então existe algum $k \in \omega$ tal que $P_{n}$ não é fechado. Portanto, existe algum $q \in \overline{P_{k}}$ tal que $q \in B_{0} \backslash \mathfrak{D}_{0}$. Note que como a topologia do produto caixa é mais fina do que a do de Tychonoff, então temos que $q \in \overline{P_{k}}$ com o fecho relativo a topologia de $\mathfrak{D}_{0}$, com o produto de Tychonoff. Podemos então escolher $p \in \mathfrak{D}_{0}$ tal que $d(p, q)<\frac{1}{2 k}$. Temos que $(p, q) \in K \subset V$, logo podemos achar $\epsilon>0$ e uma vizinhança $W$ de $q$ tal que $S(p, \epsilon) \times W \subset V$.

Como todo aberto no produto de Tychonoff é aberto no produto caixa, temos que $W \cap S(q, 1 / 2 k)$ é vizinhança de $q$. De tal forma, podemos encontrar $r \in P_{k}$ tal que $r \in W \cap S(q, 1 / 2 k)$. Desta forma, vamos mostrar que $(p, r) \in$ $U \cap V$, o que completa o resultado de que $\mathfrak{D}_{0} \times B_{0}$ não é normal. Note que $(r, r) \in H \subset U$. Logo $S(r, 1 / k) \times\{r\} \subset U$, do que se concluí que $(p, r) \in U$, visto que, pela desigualdade triangular, temos $d(p, r)<d(p, q)+d(q, r)<\frac{1}{k}$. Por outro lado, temos que $(p, r) \in V$, pois $(p, r) \in S(p, \epsilon) \times W \subset V$. 


\section{Apêndice A}

\section{Uniformidades e o produto caixa uniforme}

Como citamos em diversas partes do texto, uma das principais ferramentas para se trabalhar com o problema da paracompacidade de produtos caixa é o conceito de uniformidade.

O objetivo deste apêndice é fornecer uma leve introdução ao conceito de uniformidades, apresentando definições e resultados básicos, além de ilustrar a relação das uniformidades com produtos caixa e paracompacidade.

\section{A.1 Uniformidades}

O conceito de topologia tem origem nos espaços métricos, tendo inicialmente o objetivo de generalizar propriedades relacionadas ao conceito de vizinhança, como por exemplo convergência de sequências e continuidade de funções. De maneira semelhante, o conceito de uniformidade também se originou nos espaços métricos, porém este expressa o conceito de proximidade por meio de relações, com as quais podemos generalizar a convergência uniforme de sequência e as funções uniformemente contínuas.

Dado um espaço métrico $(M, d)$, podemos descrever as bolas deste espaço atravéz das relações $D_{\varepsilon}=\{(x, y) \in M \times M: d(x, y)<\varepsilon\}$, para cada $\varepsilon \in \mathbb{R}_{+}$. Assim, cada bola $B_{\varepsilon}\left(x_{0}\right)$ em $M$ corresponde à imagem do ponto $x_{0}$ 
pela relação $D_{\varepsilon}$.

Notação. Seja $X$ um conjunto e $D, C \subset X \times X$. Denotamos:

- a imagem de $A \subset X$ pela relação $D$ por

$$
D[A]=\{y \in X: \exists x \in A,(x, y) \in D\} .
$$

No caso em que $A=\{x\}$, escrevemos $D[\{x\}]=D[x]$

- a inversa de $D$ por $D^{-1}=\{(y, x) \in X \times X:(x, y) \in D\}$

- a composta de C com D por

$$
C \circ D=\{(x, z) \in X \times X: \exists y \in X,(x, y) \in C,(y, z) \in D\}
$$

- a diagonal de $X$ por $\Delta=\{(x, x): x \in X\}$

Esta notação de composição segue o sentido inverso à composição usual de funções. No entanto, utilizaremos tal definição pois ela se comporta de maneira mais natural com os pares ordenados.

Introduzimos então a definição de uniformidade.

Definição A.1.1. Seja $X$ um conjunto. Dizemos que $\mathfrak{D} \subset \mathcal{P}(X \times X)$ é uma uniformidade sobre $X$ se satisfaz:

1. $\mathfrak{D}$ é filtro sobre $X \times X$

2. $\Delta \subset \bigcap \mathfrak{D}$

3. $\forall D \in \mathfrak{D}, \exists C \in \mathfrak{D}, C \circ C \subset D$

4. $\forall D \in \mathfrak{D}, \exists C \in \mathfrak{D}, C^{-1} \subset D$

Um conjunto munido de uma uniformidade é chamado de espaço uniforme.

Definição A.1.2. 
- Uma base de filtro com as propriedades de 2 a 4 é chamado base uniforme sobre $X$, uma vez que o filtro gerado por tal base é uma uniformidade sobre $X$.

- Em ambos os casos, omitiremos o "sobre X" quando estiver subentendido o conjunto ao qual nos referimos.

Exemplo A.1.3. Assim como no caso das topologias, dado um conjunto $X$ temos duas uniformidades triviais sobre $X: A$ uniformidade discreta, dada pelo filtro gerado pela diagonal (ou seja, a família $\{D \in X \times X: \Delta \subset D\}$ ), a qual é a maior uniformidade em $X$; e a uniformidade caótica, dada apenas por $\{X \times X\}$.

Exemplo A.1.4. Seja $(M, d)$ um espaço métrico. A métrica d gera uma base uniforme sobre $M$, dada por todos os conjuntos $D_{\varepsilon}=\{(x, y) \in M \times M$ : $d(x, y)<\varepsilon\}$ para cada $\varepsilon>0$.

Exemplo A.1.5. Dado um grupo topológico $(G, *, \tau)$, existem 2 uniformidades compatíveis canônicas associadas a ele: uma "à direita" e outra "à esquerda". A "à direita" é dada pelas relações do tipo

$$
D_{N}=\left\{(x, y) \in G: x * y^{-1} \in G\right\}
$$

onde $N$ é uma vizinhança do elemento neutro do grupo G. A "à esquerda" é construída analogamente, apenas invertendo a ordem da operação *.

O exemplo A.1.5 mostra a relação entre grupos topológicos e uniformidades, sobre a qual já comentamos na observação 2.2.3.

A partir das uniformidades é possível também definir o conceito de continuidade uniforme, o qual ajuda a justificar a nomenclatura.

Definição A.1.6. Sejam $X$ e $Y$ conjuntos munidos de uniformidades $\mathfrak{D}_{X}$ e $\mathfrak{D}_{Y}$, respectivamente. Uma função $f: X \longrightarrow Y$ é dita uniformemente contínua se

$$
\forall D_{Y} \in \mathfrak{D}_{Y},\left\{(a, b) \in X \times X:(f(a), f(b)) \in D_{Y}\right\} \in \mathfrak{D}_{X}
$$


Note que, se $X$ e $Y$ são espaços métricos e $\mathfrak{D}_{X}$ e $\mathfrak{D}_{Y}$ as uniformidades do exemplo A.1.4, a definição acima coincide com o conceito de função uniformemente contínua em um espaço métrico. As funções uniformemente contínuas desempenham para os espaços uniformes a mesma função que as funções contínuas exercem para os espaços topológicos. De fato, elas constituem os morfismos da categoria dos espaços uniformes.

\section{A.2 Topologia induzida}

Vamos agora construir a topologia de um espaço uniforme:

Definição A.2.1. Dado um espaço uniforme, definimos

$$
\tau(\mathfrak{D})=\{G \subset X: \forall x \in G, \exists D \in \mathfrak{D}, D[x] \subset G\}
$$

Note que, se fixarmos $\mathfrak{D}_{d}$ como a uniformidade do exemplo A.1.4, temos que $\tau\left(\mathfrak{D}_{d}\right)$ nada mais é do que a topologia gerada pela métrica $d$. Da mesma forma que em espaços métricos, temos que, dado um espaço uniforme $(X, \mathfrak{D})$, o conjunto $\tau(\mathfrak{D})$ é uma topologia sobre $X$. Chamamos-lo então de topologia induzida por $\mathfrak{D}$. No caso em que $\mathfrak{D}$ é uma base uniforme, por topologia induzida por $\mathfrak{D}$ nos referimos à topologia induzida pela uniformidade gerada pela base $\mathfrak{D}$.

A seguinte proposição, em [Kel75], caracteriza interior e fecho na topologia induzida.

Proposição A.2.2 (Interior e fecho em uniformidades). Seja $(X, \mathfrak{D})$ um espaço uniforme equipado com a topologia induzida por $\mathfrak{D}$. Dado $A \subset X$, temos:

- o interior de $A$ é $\{x \in A: \exists D \in \mathfrak{D}, D[x] \subset A\}$

- o fecho de $A$ é $\bigcap\{D[A]: D \in \mathfrak{D}\}$

Demonstração. 
- Definimos $B=\{x \in A: \exists D \in \mathfrak{D}, D[x] \subset A\}$. Seja $O \subset A$ aberto e $p \in O$. Como $O$ é aberto, podemos encontrar um $D \in \mathfrak{D}$ tal que $D[x] \subset O \subset A$, de modo que $p \in B$ e, portanto, $O \subset B$. Assim, basta provar que $B$ é aberto. Seja $x \in B$. Então existe $D \in \mathfrak{D}$ tal que $D[x] \subset A$. Seja $C \in \mathfrak{D}$ tal que $C \circ C \subset D$. Dado $y \in C[x]$, temos $C[y] \subset(C \circ C)[x] \subset D[x] \subset A$. Assim, $C[x] \subset B$, logo $B$ é de fato aberto.

- Note que dado $x \in X,\{D[x]: D \in \mathfrak{D}\}$ é um sistema fundamental de vizinhanças de $x$. Então podemos dizer que $x \in \bar{A}$ se e somente se para todo $D \in \mathfrak{D}, D[x]$ intercepta $A$. Note que $D[x]$ intersepta $A$ se e somente se $x \in D^{-1}[A]$. Contudo, para cada $D \in \mathfrak{D}$, temos que $D^{-1} \in \mathfrak{D}$. Então $x \in \bar{A}$ se e somente se para todo $D \in \mathfrak{D}, x \in D[A]$, como queríamos.

\section{Definição A.2.3.}

- Seja $(X, \tau)$ um espaço topológico. Uma uniformidade (base uniforme) $\mathfrak{D}$ sobre $X$ é dita compatível com $X$ se $\tau(\mathfrak{D})=\tau$.

- Um espaço topológico $X$ é dito uniformizável se existe uma uniformidade compativel com $X$.

Proposição A.2.4. Seja $(X, \tau)$ um espaço topológico e $\mathfrak{D}$ uma uniformidade compativel com X. São equivalentes:

1. X é Hausdorff

2. $\cap \mathfrak{D}=\Delta$

Neste caso, dizemos que $\mathfrak{D}$ é Hausdorff.

Demonstração.

- (1) $\Longrightarrow(2)$ : Para esta implicação, basta supor $X$ espaço $T_{1}$ (por simetria, pode-se diminuir a hipótese para $T_{0}$, mas a escrita fica desnecessariamente carregada). Pela definição de uniformidade, temos que $\Delta \subset$ 
$\bigcap \mathfrak{D}$. Para completar a igualdade, suponha que exista $(x, y) \in \bigcap \mathfrak{D}$ tal que $x \neq y$. Como $X$ é $T_{1}$, escolhemos um aberto $V$ tal que $x \in V$ e $y \notin V$. Como $\mathfrak{D}$ é compatível com $X$, existe uma relação $D \in \mathfrak{D}$ tal que $D[x] \subset V$. Temos assim que $y \notin D[x]$. Portanto $(x, y) \notin D$, o que contraría a hipótese de que $(x, y) \in \bigcap \mathfrak{D}$. Logo, $\bigcap \mathfrak{D}=\Delta$

- $(2) \Longrightarrow(1)$ : Seja $x \in X$. Defino $W_{x}=\{F \subset X: F$ é vizinhança fechada de $x\}$. Note que, como o conjunto de todos os $D[x]$, com $D \in \mathfrak{D}$, é um sistema fundamental de vizinhanças de $\mathrm{x}$, temos que $\bigcap W_{x}=\bigcap W_{x}^{\prime}$, onde $W_{x}^{\prime}=\{\overline{D[x]}: D \in \mathfrak{D}\}$. Pela proposição A.2.2 temos que, dado $D \in \mathfrak{D}, \overline{D[x]}=\bigcap\left\{D^{\prime}[D[x]]: D^{\prime} \in \mathfrak{D}\right\}$. Seja $y \in \bigcap W_{x}^{\prime}$, pela definição e pelas propriedades já descritas, vale:

$$
\forall D, D^{\prime} \in \mathfrak{D}, \exists z \in D[x], y \in D^{\prime}[z]
$$

ou seja,

$$
\forall D, D^{\prime} \in \mathfrak{D},(x, y) \in D \circ D^{\prime}
$$

Usemos agora a hipótese de que $\bigcap \mathfrak{D}=\Delta$. Suponhamos $x \neq y$. Temos que $\exists D \in \mathfrak{D}$ tal que $(x, y) \notin D$. Pela definição de uniformidade, existe $C \in \mathfrak{D}$ tal que $C \circ C \subset D$. Logo $(x, y) \notin C \circ C$, o que contraria (A.1), $\operatorname{logo} x=y$. Portanto, temos $\bigcap W_{x}=\{x\}$, para cada $x \in X$, o que é equivalente a " $X$ é Hausdorff".

A maioria dos teoremas sobre produto caixa tem como hipótese que os espaços em questão sejam regulares. Portanto, é de se esperar que os espaços uniformizáveis sejam $T_{3}$. Mais do que isso, os espaços uniformizáveis são os exatamente os espaços $T_{3 \frac{1}{2}}$, como mostra o próximo teorema. Utilizaremos a demonstração em [Per64].

Teorema A.2.5. Um espaço topológico $X$ é $T_{3 \frac{1}{2}}$ se e somente se for uniformizável. 
Demonstração. Seja $(X, \tau)$ um espaço $T_{3 \frac{1}{2}}$. Vamos mostrar que $X$ é uniformizável. Para cada $\phi: X \longrightarrow \mathbb{I}$ contínua e cada $\epsilon>0$, definimos

$$
u_{\phi, \epsilon}=\{(x, y) \in X \times X:|\phi(x)-\phi(y)|<\epsilon\} .
$$

Seja $\mathfrak{B}=\left\{u_{\phi, \epsilon}: \epsilon \in \mathbb{R}_{+}^{*}, \phi \in C(X, \mathbb{I})\right\}$. Vamos mostrar que $\mathfrak{U}=\left\{\cap B: \emptyset \neq B \in[\mathfrak{B}]^{<\omega}\right\}$ é uma base uniforme compatível com $X$. Como $\Delta \subset u_{\phi, \epsilon}$, temos que $\emptyset \notin \mathfrak{U}$ e portanto $\mathfrak{U}$ é base de filtro e satisfaz também a condição 2. Seja $\phi: X \longrightarrow \mathbb{I}$ uma função contínua e $\epsilon>0$. Pela desigualdade triangular para números reais, temos que $u_{\phi, \frac{\epsilon}{2}} \circ u_{\phi, \frac{\epsilon}{2}} \subset u_{\phi, \epsilon}$, logo a condição 3 é satisfeita. Além disso, como $u_{\phi, \epsilon}^{-1}=u_{\phi, \epsilon}$, a condição 4 também é satisfeita.

Verifiquemos que $\mathfrak{U}$ é compatível com $(X, \tau)$. Seja $A \in \tau$ e $a \in A$. Como $X$ é $T_{3 \frac{1}{2}}$, podemos construir uma função contínua $f: X \longrightarrow \mathbb{I}$ tal que $f(a)=$ 0 e $f(y)=1$ para todo $y \in X \backslash A$. Temos então que $u_{f, 1}[a] \subset A$, e portanto, $A \in \tau(\mathfrak{U})$. Seja agora $B \in \tau(\mathfrak{U})$ e fixe $b \in B$. Podemos encontrar $\phi: X \longrightarrow \mathbb{I}$ contínua e $\epsilon>0$ tais que $u_{\phi, \epsilon}[b] \subset B$. Como $\phi$ é contínua, podemos encontrar um aberto $V \in \tau$ tal que $x \in V \subset \phi^{-1}(] \phi(b)-\epsilon, \phi(b)+\epsilon[) \subset u_{\phi, \epsilon}[b] \subset B$. Desta forma, temos que $B$ é aberto em $X$. Concluímos assim que $(X, \tau)$ é uniformizável.

Vamos agora verificar a reciproca. Seja $(X, \mathfrak{D})$ um espaço uniforme. Vamos mostrar que $(X, \tau(\mathfrak{D}))$ é $T_{3 \frac{1}{2}}$. Fixemos $F \subset X$ fechado e $z \in X \backslash F$. Podemos encontrar $C \in \mathfrak{D}$ simétrico (isto é, $C=C^{-1}$ ) tal que $C[z] \cap F=\emptyset$. Definimos então por indução uma sequência $\left(u_{n}\right)_{n \in \omega}$ tal que $u_{0}=C$ e, para cada $n \in \omega, u_{n}$ é simétrico e temos $u_{n+1} \circ u_{n+1} \subset u_{n}$.

Chamamos de racional diádico um número racional cuja transcrição em base binária possui comprimento finito. Descreveremos um racional diádico $r \in] 0,1]$ por $r=\sum_{n=1}^{K} 2^{-r_{n}}$, onde $\left(r_{n}\right)_{0<n \leq K}$ é uma sequência finita crescente de naturais. Para cada tal $r$, definimos

$$
v_{r}=u_{n_{1}} \circ u_{n_{2}} \circ \cdots \circ u_{n_{K-1}} \circ u_{n_{K}}
$$

Note que no caso particular em que $r=0$, temos $v_{1}=u_{0}$. Definimos $v_{0}=\Delta$. Vamos mostrar que $v_{r} \subset v_{r^{\prime}}$ para quaisquer $r, r^{\prime}$ racionais diádicos 
tais que $0 \leq r \leq r^{\prime} \leq 1$. Para tanto, vamos demonstrar a seguinte afirmação.

Afirmação. $\forall n \in \omega, \forall m \leq 2^{n-1}, v_{m 2^{-n}} \circ u_{n} \subset v_{(m+1) 2^{-n}}$

Procedemos por indução em $n$. Para $n=0$, temos $v_{0} \circ u_{0}=v_{0} \circ \Delta=$ $v_{0}=v_{1}$. Suponha a afirmação válida para $n=k-1>0$. Consideramos primeiro o caso em que $m$ é par. Seja $m=2 p$, com $p \in \omega$. Temos assim, $m 2^{-n}=(2 p) 2^{-n}=p 2^{-(n-1)}$ e $(m+1) 2^{-n}=(2 p+1) 2^{-n}=p 2^{-(n-1)}+2^{-n}$. Seguindo a definição, temos

$$
v_{(m+1) 2^{-n}}=v_{p 2^{-(n-1)}+2^{-n}}=v_{p 2^{-(n-1)}} \circ u_{n}=v_{m 2^{-n}} \circ u_{n},
$$

como gostaríamos.

Suponhamos agora que $m$ seja um número ímpar. Seja $m=2 p+1$. Temos assim $m 2^{-n}=p 2^{-(n+1)}+2^{-n}$ e $(m+1) 2^{-n}=(p+1) 2^{-(n-1)}$. Pela hipótese de indução, temos:

$$
v_{p 2^{-(n-1)}} \circ u_{n-1} \subset v_{(p+1) 2^{-(n-1)}}
$$

Usando o fato de que $u_{n} \circ u_{n} \subset u_{n-1}$, temos:

$$
\begin{aligned}
v_{m 2^{-n}} \circ u_{n} & =v_{p 2^{-(n-1)}+2^{-n}} \circ u_{n} \\
& =v_{p 2^{-(n-1)}} \circ u_{n} \circ u_{n} \\
& \subset v_{p 2^{-(n-1)}} \circ u_{n-1} \\
& \subset v_{(p+1) 2^{-(n-1)}}=v_{(m+1) 2^{-n}}
\end{aligned}
$$

como gostaríamos, completando assim a demonstração da afirmação.

Sejam $r$ e $r^{\prime}$ racionais diádicos tais que $0 \leq r \leq r^{\prime} \leq 1$, vamos mostrar que $v_{r} \subset v_{r^{\prime}}$. Podemos escrever tais frações em um denominador comum, de maneira que $r=a 2^{-k}$ e $r=b 2^{-k}$. Como todo $u_{n}$ contém a diagonal $\Delta$, pela afirmação temos:

$$
v_{r} \subset v_{a 2^{-k}} \circ u_{k} \subset v_{(a+1) 2^{-k}} \circ u_{k} \subset \ldots \subset v_{b 2^{-k}} \circ u_{k}=v_{r^{\prime}}
$$

Vamos agora construir uma função $\phi: X \longrightarrow \mathbb{I}$ definida por $\phi(z)=0$ e 
por

$$
\phi(x)=\sup \left\{r: x \notin u_{r}[z]\right\}
$$

para $x \neq z$. Por construção, como $u_{0}[z] \subset X \backslash F$, temos que, dado $x \in F$, $x \notin u_{0}[z]=v_{1}[z]$, e portanto $\phi(x)=1$. Resta apenas demonstrar que $\phi$ é contínua. Para isso, fixemos $x \in X$ e $\epsilon>0$. Seja $N \in \omega$ tal que $2^{N}>\frac{2}{\epsilon}$. Vamos mostrar que $u_{N}[x]$ é uma vizinhança de $x$ a qual testemunha a continuidade de $\phi$, isto é, $\left.\phi\left(u_{n}[x]\right) \subset\right] \phi(x)-\epsilon, \phi(x)+\epsilon[$.

Seja $y \in u_{N}[x]$. Temos $(x, y) \in u_{N}$ e, pela simetria de $u_{N}$, temos também $(y, x) \in u_{N}$. Suponhamos que $\phi(x), \phi(y)<1$. Assim, podemos escolher naturais $m, m^{\prime}>0$ tais que $m 2^{-N}>\phi(x) \geq(m-1) 2^{N} \mathrm{e}$ $m^{\prime} 2^{-N}>\phi(y) \geq\left(m^{\prime}-1\right) 2^{-N}\left(m 2^{-N}\right.$ e $m^{\prime} 2^{-N}$ são aproximações à $N$ ésima "casa" da decomposição binária de $\phi(x)$ e $\phi(y)$, respectivamente). Pela definição de $\phi$, temos que $x \in v_{m 2^{-N}}[z]$ e $y \in v_{m^{\prime} 2^{-N}}[z]$, de maneira que $(z, x) \in v_{m 2^{-N}}[z]$ e $(z, y) \in v_{m^{\prime} 2^{-N}}[z]$. Usando a composição e a afirmação já demonstrada, temos:

$$
(z, y) \in v_{m 2^{-N}} \circ u_{N} \subset v_{(m+1)} 2^{-N} \mathrm{e}(z, x) \in v_{m^{\prime} 2^{-N}} \circ u_{N} \subset v_{\left(m^{\prime}+1\right)} 2^{-N} .
$$

Pela definição de $\phi$, temos $\phi(y)<(m+1) 2^{-N}$ e $\phi(x)<\left(m^{\prime}+1\right) 2^{-N}$. Desta maneira, temos

$$
|\phi(x)-\phi(y)| \leq 2\left(2^{-N}\right)<2\left(\frac{\epsilon}{2}\right)=\epsilon .
$$

No caso em que $\phi(x)=1$ ou $\phi(y)=1$, podemos encontrar $r \in] 1-$ $2^{-(N+1)}, 1\left[\right.$ diádico tal que $x \in v_{r}[z]$ ou $y \in v_{r}[z]$, respectivamente. Utilizamos então a mesma aproximação da decomposição binária para $r$ e procedemos da mesma maneira.

Concluímos assim que $\left.\phi\left(u_{n}[x]\right) \subset\right] \phi(x)-\epsilon, \phi(x)+\epsilon[$ e, portanto, $\phi$ é contínua, como gostaríamos.

Note que este resultado, junto com a proposição A.3.4, garante que o produto caixa preserva a propriedade de Tychonoff (observação 2.2.3). 
O conceito de completude também pode ser expresso em termos de uniformidades, através de filtros.

Definição A.2.6. Seja $X$ um espaço topológico e $\mathfrak{D}$ uma uniformidade compativel sobre $X$.

1. Um filtro $\mathfrak{F}$ em $X$ é dito $\mathfrak{D}$-Cauchy se vale

$$
\forall D \in \mathfrak{D}, \exists x \in X, D[x] \in \mathfrak{F}
$$

2. $\mathfrak{D}$ é dito completo se todo filtro $\mathfrak{D}$-Cauchy $\mathfrak{F}$ converge em $X$ (isto é, existe um $x \in X$ tal que todas as vizinhanças de $x$ pertencem a $\mathfrak{F}$ ).

3. Um espaço topológico é dito topologicamente completo se ele possui uma uniformidade completa compativel.

Outro conceito que pode ser definido para uniformidades é a $\kappa$-metrizabilidade (definição 1.2.30).

Definição A.2.7. Seja $\kappa$ um cardinal regular infinito. Um espaço $X$ é dito $\kappa$-metrizável se $X$ possui uma base uniforme compativel $\mathfrak{D}$ tal que $(\mathfrak{D}, \subset)$ tenha tipo de ordem $\kappa$.

Em [Wil84], Scott Williams demonstra o teorema 1.2.31 para esta definição de $\kappa$-metrizabilidade. No mesmo artigo, novamente usando uniformidades, é apresentada uma versão mais completa do teorema 3.1.5, a qual diminui a hipótese de compacidade para paracompacidade e compacidade local.

Teorema A.2.8 (Nabla Lemma). Sejam $X_{i}$ espaços topológicos $T_{3}$, paracompactos e localmente compactos para cada $i \in I$. Então, são equivalentes:

1. $\square_{i \in I} X_{i}$ é paracompacto.

2. $\nabla_{i \in I} X_{i}$ é paracompacto.

3. $\nabla_{i \in I} K_{i}$ é paracompacto, se para todo $i \in I$ tivermos $K_{i}$ subespaço compacto de $X_{i}$. 
Uma propriedade das uniformidades, a qual as tornam ferramentas para se estudar produtos caixa, é o fato de que podemos definir paracompacidade através de uniformidades.

Definição A.2.9. Sejam $X$ um espaço topológico, $A, B \subset \mathcal{P}(X)$ famílias de subconjuntos de $X$ e $D \subset X \times X$ uma relação em $X$. Dizemos que $D$ refina $A$ se tivermos que a familia $\{D[x]: x \in X\}$ refina $A$, ou seja, se $\forall x \in X, \exists a_{x} \in A, D[x] \subset a_{x}$.

Definição A.2.10. Dado um espaço topológico $X$ e uma relação $D \subset X \times X$, dizemos que $D$ é uma entourage se $D$ for uma vizinhança da diagonal $\Delta \subset$ $X \times X$, ou seja, se existe um conjunto $V \subset X \times X$ aberto na topologia produto tal que $\Delta \subset V \subset D$.

Observação A.2.11. Dado um espaço uniforme $(X, \mathfrak{D})$, se equiparmos $X$ com a topologia induzida por $\mathfrak{D}$, e em seguida munirmos $X \times X$ com a topologia produto, temos que todos os elementos de $\mathfrak{D}$ são entourages de $X$. Por isso, muitos autores usam o termo entourage com o sentido de elemento de uma uniformidade. A recíproca desta afirmação (ou seja, dado um espaço topológico, o conjunto de todas as entourages forma uma uniformidade sobre X) necessita da hipótese de que o espaço é paracompacto. Tal recíproca será demonstrada no lema A.2.15.

Definição A.2.12. Dado um espaço topológico $X$, dizemos que uma cobertura aberta de $X$ é impar se existe uma entourage que a refine.

Teorema A.2.13. Seja X um espaço topológico regular. Temos então:

$X$ é paracompacto $\Longleftrightarrow$ Toda cobertura aberta de X é impar.

Para provar essa equivalência, usaremos o seguinte conceito:

\section{Definição A.2.14.}

- Seja $X$ um espaço topológico e $\mathcal{A}$ uma cobertura de $X$. Dado $M \subset X$, definimos o conjunto estrela de $M$ por $\operatorname{St}(M, \mathcal{A})=$ $\bigcup\{A \in \mathcal{A}: A \cap M \neq \emptyset\}$. No caso $M=\{x\}$, denotamos $S t(\{x\}, \mathcal{A})=$ $\operatorname{St}(x, \mathcal{A})$. 
- Dada uma outra cobertura $\mathcal{B}$ de $X$, dizemos que $\mathcal{B}$ é refinamento baricêntrico de $\mathcal{A}$ se para cada $x \in X$ existe um $A \in \mathcal{A}$ tal que $\operatorname{St}(x, \mathcal{B}) \subset$ $A$.

Demonstração do teorema A.2.13.

- $[\Leftarrow]$ Seja $\mathcal{V}$ uma cobertura aberta de $X$. Seja $D$ uma entourage que refina $\mathcal{V}$. Para cada $x \in X$ existe um aberto $W_{x}$ tal que $(x, x) \in$ $W_{x} \times W_{x} \subset D$. Vou mostrar que $\mathcal{W}=\left\{W_{x}: x \in X\right\}$ é um refinamento baricêntrico de $\mathcal{V}$. Como $D$ refina $\mathcal{V}$, então para cada $x \in X$ existe $V \in \mathcal{V}$ tal que $D[x] \subset V$ Basta então mostrarmos que $\forall x \in$ $X, S t(x, \mathcal{W}) \subset D[x]$. Seja $p \in S t(x, \mathcal{W})$. Existe $y \in X$ tal que $\{p, x\} \subset W_{y}$. Como $W_{y} \times W_{y} \subset D$, então $(x, p) \in D$. Logo, $p \in D[x]$, como queríamos. Para concluir este lado da demonstração, observamos que se toda cobertura aberta de um espaço tiver um refinamento baricêntrico então esse espaço é paracompacto (para este resultado, veja por exemplo [Eng89] ou [Kel75]).

- $[\Rightarrow]$ Suponha $X$ paracompacto. Seja $\mathcal{V}$ cobertura aberta de $X$. Como $X$ é regular, podemos refinar $\mathcal{V}$ com uma cobertura aberta $\mathcal{U}$ cujos fechos de seus elementos estejam contidos em elementos de $\mathcal{V}$. Como $X$ é paracompacto, podemos refinar $\mathcal{U}$ por uma cobertura localmente finita. Note que os fechos desse refinamento formam um refinamento fechado localmente finito de $\mathcal{V}$. Chamaremos tal refinamento de $\mathcal{A}$. Para cada $A \in \mathcal{A}$, existe um $V_{A} \in \mathcal{V}$ tal que $A \subset V_{A}$. Definimos:

$$
W_{A}=\left(V_{A} \times V_{A}\right) \cup((X \backslash A) \times(X \backslash A)) .
$$

Note que $W_{A}$ é aberto em $X \times X$ e que $\Delta \subset W_{A}$. Além disso, temos que se $x \in A$, então $W_{A}[x]=V_{A}$. Definimos então:

$$
D=\bigcap\left\{W_{A}: A \in \mathcal{A}\right\}
$$

Vamos mostrar que $D$ é o refinamento ímpar de $\mathcal{V}$ que queremos. Para cada $x \in X$, temos $D[x] \subset W_{A}[x] \subset V_{A} \in \mathcal{V}$, logo $D$ refina $\mathcal{V}$. Falta 
mostrar que $D$ é entourage. Como $\forall A \in \mathcal{A}, \Delta \subset W_{A}$, então $\Delta \subset$ $\bigcap_{A \in \mathcal{A}} W_{A}$. Agora, seja $x \in X$. Como $\mathcal{A}$ é localmente finita, existe $O_{x}$ vizinhança aberta de $x$ que intercepta apenas uma quantidade finita de elementos de $\mathcal{A}$. Se $A \in \mathcal{A}$ é tal que $A \cap O_{x}=\emptyset$, então $O_{x} \subset$ $X \backslash A$, portanto $O_{x} \times O_{x} \subset W_{A}$. Assim, temos que $\left(O_{x} \times O_{x}\right) \cap D=$ $\left(O_{x} \times O_{x}\right) \cap\left(\bigcap\left\{W_{A}: A \cap O_{x} \neq \emptyset\right\}\right)$ é uma interseção de abertos, logo é uma vizinhança aberta de $(x, x)$. Então $D$ é de fato entourage, como queríamos.

Apresentamos agora uma aplicação interessante dessa equivalência:

Lema A.2.15. Se X é um espaço paracompacto Hausdorff, então o conjunto $\mathcal{N}$ de todas as entourages de X é uma uniformidade completa compativel com $X$.

Demonstração. Pela definição de vizinhança, fica fácil ver que $\mathcal{N}$ é filtro em $X \times X$.

Seja $D \in \mathcal{N}$. Para cada $x \in X$, podemos escolher $G_{x}$ uma vizinhança de $x$ tal que $G_{x} \times G_{x} \subset D$. Como $X$ é paracompacto, pelo teorema A.2.13, podemos refinar a cobertura $\left\{G_{x}: x \in X\right\}$ de $X$ por uma entourage $E \in \mathcal{N}$. Definimos $C=E \cap E^{-1}$. Como $E^{-1} \in \mathcal{N}$, então $C \in \mathcal{N}$. Note que $E \subset D$, pois $\forall x \in X, E[x] \subset G_{x} \subset D[x]$. Como $C$ é simétrico, temos que $C^{-1} \subset D$. Vamos mostrar agora que $C \circ C \subset D$. Sejam $(x, y),(y, z) \in C$. Note que $x, y \in C[y]$. Existe um $w \in X$ tal que $C[y] \subset G_{w}$, já que $C \subset E$. Como $G_{w} \times G_{w} \subset D$, então $(x, z) \in D$. Logo, $\mathcal{N}$ é de fato uma uniformidade.

Vamos mostrar que $\mathcal{N}$ é compatível com $X$. Dado $x \in X$ e $D \in \mathcal{N}$, existe um aberto $V \subset X$ tal que $(x, x) \in V \times V \subset D \times D$. Note que $(V \times V)[x]=V$, o qual é aberto, portanto temos $x \in V \subset D[x]$, ou seja, $D[x]$ é vizinhança de $x$. Por outro lado, seja $G \subset X$ uma vizinhança aberta de $x$. Definimos:

$$
F=(G \times G) \cup((X \backslash\{x\}) \times(X \backslash\{x\}))
$$

assim, como $X$ é Haussdorf, temos que $\{x\}$ é fechado, logo $F$ é aberto. Como 
$\Delta \subset F$, temos que $F \in \mathcal{N}$, e como $F[x] \subset G$, concluímos que $\mathcal{N}$ é compatível $\operatorname{com} X$.

Por fim, para provar que todo filtro $\mathcal{N}$-Cauchy converge em $X$, vamos demonstrar a contrapositiva. Seja $\mathcal{F}$ um filtro não convergente em $X$. Então, para cada $x \in X$ existe uma vizinhança aberta $G_{x}$ tal que $G_{x} \notin \mathcal{F}$. Como $X$ é paracompacto, pelo teorema A.2.13, existe $D \in \mathcal{N}$ que refina a cobertura $\left\{G_{x}: x \in X\right\}$. Temos então que $\forall X \in X, D[x] \notin \mathcal{F}$, logo $\mathcal{F}$ não é $\mathcal{N}$ Cauchy, como queríamos.

\section{A.3 Produtos}

A partir de uniformidades, podemos definir tanto o produto de Tychonoff quanto o produto caixa [Bel11].

Definição A.3.1. Para cada $i \in I$, seja $X_{i}$ munido de uma uniformidade $\mathfrak{D}_{i}$. Para cada $j \in I$ e cada $D \in \mathfrak{D}_{j}$, definimos:

$$
\widehat{D}=\left\{(x, y) \in\left(\prod_{i \in I} X_{I}\right) \times\left(\prod_{i \in I} X_{I}\right):\left(x_{j}, y_{j}\right) \in D\right\}
$$

Seja $\mathfrak{P}=\left\{\widehat{D}: j \in I, D \in \mathfrak{D}_{j}\right\}$. Definimos o produto de Tychonoff das uniformidades $\left(\mathfrak{D}_{i}\right)_{i \in I}$ por $\mathrm{T}_{i \in I} \mathfrak{D}_{i}=\left\{\bigcap P: P \in[\mathfrak{P}]^{<\omega}, P \neq \emptyset\right\}$

Proposição A.3.2. Para cada $i \in I$, seja $X_{i}$ um espaço topológico munido de uma uniformidade compativel $\mathfrak{D}_{i}$. Então $\mathrm{T}_{i \in I} \mathfrak{D}_{i}$ é uma base uniforme compativel com o produto de Tychonoff $\prod_{i \in I} X_{i}$

Demonstração. Inicialmente, vamos verificar que $\mathrm{T}_{i \in I} \mathfrak{D}_{i}$ é uma base uniforme sobre $\prod_{i \in I} X_{i}$. Note que $\mathrm{T}_{i \in I} \mathfrak{D}_{i}$ é uma base de filtro. Seja $\Delta$ a diagonal de $\prod_{i \in I} X_{i}$. Qualquer $\widehat{D} \in \mathfrak{P}$ satisfaz $\Delta \subset \widehat{D}$, de modo que $\Delta \subset \bigcap P$. Portanto $\emptyset \notin \mathrm{T}_{i \in I} \mathfrak{D}_{i}$. Por construção, temos que $\prod_{i \in I} X_{i}$ é fechado para intersecções finitas.

Já mostramos que $\mathrm{T}_{i \in I} \mathfrak{D}_{i}$ satisfaz a condição 2. Para verificar as condições 3 e 4 , fixemos $A \in \mathrm{T}_{i \in I} \mathfrak{D}_{i}$. Definimos $P=\left\{\widehat{D}_{n} \in \mathfrak{D}_{i_{n}}: 0 \leq n<k\right\} \in$ $[\mathfrak{P}]^{<\omega}$ de maneira que $A=\bigcap P$. Para cada $n<k$, podemos encontrar 
$B_{n}, C_{n} \in \mathfrak{D}_{i_{n}}$ tais que $B_{n} \circ B_{n} \subset D_{n}$ e $C_{n}{ }^{-1} \subset D_{n}$. Definindo $B=\bigcap_{n<k} \widehat{B_{n}} \in$ $\mathrm{T}_{i \in I} \mathfrak{D}_{i}$ e $C=\bigcap_{n<k} \widehat{C_{n}} \in \mathrm{T}_{i \in I} \mathfrak{D}_{i}$, temos $B \circ B \subset A$ e $C^{-1} \subset A$, como gostaríamos.

Vamos mostrar que tal base uniforme é compatível com o produto de Tychonoff $\prod_{i \in I} X_{i}$

Seja $V$ um aberto básico de $\prod_{i \in I} X_{i}$ tal que $\operatorname{spt}(V)=1$. Vamos mostrar que $V$ é aberto na topologia induzida por $\mathrm{T}_{i \in I} \mathfrak{D}_{i}$. Seja $p \in V$, e seja $j \in I$ tal que $V_{j} \neq X_{j}$. Como $\mathfrak{D}_{j}$ é compatível com $X_{j}$, podemos encontrar $D \in \mathfrak{D}_{j}$ tal que $D\left[p_{j}\right] \subset V_{j}$. Temos então que $\bar{D}[p] \subset V$, portanto $V \in \tau\left(\mathrm{T}_{i \in I} \mathfrak{D}_{i}\right)$, como queríamos.

Considere agora $U \in \tau\left(\mathrm{T}_{i \in I} \mathfrak{D}_{i}\right)$. Vamos mostrar que $U$ é aberto no produto de Tychonoff $\prod_{i \in I} X_{i}$. Seja $q \in U$. Podemos encontrar então $P \in$ $[\mathfrak{P}]^{<\omega}$ tal que $(\bigcap P)[q] \subset U$. Seja $P=\left\{\widehat{D}_{n} \in \mathfrak{D}_{i_{n}}: 0 \leq n<k<\omega\right\}$. Para cada $n<k$, temos que $D_{n}\left[q_{i_{n}}\right] \subset U_{i_{n}}$ é uma vizinhança de $q_{i_{n}}$. Definindo $W_{i_{n}}=D_{n}\left[q_{i_{n}}\right]$ para cada $n<k$ e $W_{i}=X_{i}$ para os demais $i \in I$, temos que $W \subset U$ é uma vizinhança de $q$, o que garante que $U$ é aberto no produto de Tychonoff $\prod_{i \in I} X_{i}$.

Definição A.3.3. Para cada $i \in I$, seja $X_{i}$ munido de uma uniformidade $\mathfrak{D}_{i}$. Para cada $D \in \prod_{i \in I} \mathfrak{D}_{i}$ definimos:

$$
\square D=\square_{i \in I} D_{i}=\left\{(x, y) \in\left(\prod_{i \in I} X_{I}\right) \times\left(\prod_{i \in I} X_{I}\right): \forall i \in I,\left(x_{i}, y_{i}\right) \in D_{i}\right\}
$$

Definimos então o produto caixa das uniformidades $\left(\mathfrak{D}_{i}\right)_{i \in I}$ por $\square_{i \in I} \mathfrak{D}_{i}=$ $\left\{\square D: D \in \prod_{i \in I} \mathfrak{D}_{i}\right\}$.

Proposição A.3.4. Seja $\mathfrak{D}_{i}$ uma uniformidade compatível com o espaço topológico $X_{i}$ para cada $i \in I$. Então $\square_{i \in I} \mathfrak{D}_{i}$ é uma base uniforme compatível com o produto caixa $\square_{i \in I} X_{i}$.

Demonstração. Verifiquemos que $\square_{i \in I} \mathfrak{D}_{i}$ é uma base uniforme. Note que $\square_{i \in I} \mathfrak{D}_{i}$ é uma base de filtro. De fato, temos $\emptyset \notin \square_{i \in I} \mathfrak{D}_{i}$ pois, caso contrário, teríamos $\emptyset \in \mathfrak{D}_{i}$ para algum $i \in I$. Dados $A, B \in \prod_{i \in I} \mathfrak{D}_{i}$, temos que $\square A \cap \square B=\square_{i \in I}\left(A_{i} \cap B_{i}\right) \in \square_{i \in I} \mathfrak{D}_{i}$. 
Vamos verificar que $\square_{i \in I} \mathfrak{D}_{i}$ satisfaz a condição 2. Sejam $D \in \prod_{i \in I} \mathfrak{D}_{i} \mathrm{e}$ $x \in \prod_{i \in I} X_{i}$. Temos que $\left(x_{i}, x_{i}\right) \in D_{i}$ para todo $i \in I$, portanto $(x, x) \in \square D$.

Para verificar a condição 3 , fixemos $\square D \in \square_{i \in I} \mathfrak{D}_{i}$. Para cada $i \in I$, escolhemos $C_{i} \in \mathfrak{D}_{i}$ tal que $C_{i} \circ C_{i} \subset D_{i}$. Definindo $C=\left(C_{i}\right)_{i \in I}$, temos que $\square C \circ \square C \subset \square D$. Da mesma forma, para verificar a condição 4, escolhemos $C_{i} \in \mathfrak{D}_{i}$ para cada $i \in I$ tal que $C_{i}^{-1} \subset D_{i}$, e assim obtemos $C=\left(C_{i}\right)_{i \in I}$ satisfazendo $(\square C)^{-1} \subset \square D$.

Vamos então mostrar que $\square_{i \in I} \mathfrak{D}_{i}$ é compatível com $\square_{i \in I} X_{i}$.

Seja $V$ aberto básico de $\square_{i \in I} X_{i}$ e fixemos $p \in V$. Como para cada $i \in I$ temos $\mathfrak{D}_{i}$ compatível com $X_{i}$, temos um $D_{i} \in \mathfrak{D}_{i}$ tal que $p_{i} \in D_{i}\left[p_{i}\right]$. Desta maneira, definindo $D=\left(D_{i}\right)_{i \in I}$, temos $\square D \in \square_{i \in I} \mathfrak{D}_{i}$, e também $\square D[p] \subset V$, $\operatorname{logo} V \in \tau\left(\prod_{i \in I} \mathfrak{D}_{i}\right)$.

Seja agora $U \in \tau\left(\prod_{i \in I} \mathfrak{D}_{i}\right)$. Vamos mostrar que $U$ é aberto em $\square_{i \in I} X_{i}$. Seja $q \in U$. Então existe $D \in \prod_{i \in I} \mathfrak{D}_{i}$ tal que $\square D[q] \subset U$. Para cada $i \in I$, temos que $D_{i}$ temos $D_{i}\left[q_{i}\right]$ uma vizinhança de $q_{i}$, $\operatorname{logo} \square_{i \in I} D_{i}\left[q_{i}\right]=$ $\square D[q] \subset U$ é uma vizinhança de $q$. Assim, $U$ é aberto em $\square_{i \in I} X_{i}$, como gostaríamos.

No caso específico do produto de $\omega$ fatores iguais, podemos através das uniformidades construir um produto intermediário entre o produto de Tychonoff e o produto caixa. Tal produto é conhecido como produto caixa uniforme.

Proposição A.3.5. Seja $X$ um conjunto munido de uma uniformidade $\mathfrak{D}$. Para cada $D \in \mathfrak{D}$, seja:

$$
\bar{D}=\left\{(x, y) \in\left(\prod^{\omega} X\right) \times\left(\prod^{\omega} X\right): \forall i \in \omega,\left(x_{i}, y_{i}\right) \in D\right\}
$$

Então o conjunto $\overline{\mathfrak{D}}=\{\bar{D}: D \in \mathfrak{D}\}$ é uma base uniforme sobre $\prod^{\omega} X$.

O conjunto $\overline{\mathfrak{D}}$ é chamado base uniforme constante. O conjunto $\prod^{\omega} X$ equipado com a topologia induzida por $\overline{\mathfrak{D}}$ é chamado de produto caixa uniforme.

Demonstração. Vamos mostrar que $\overline{\mathfrak{D}}$ é uma base de filtro. Temos que $\emptyset \notin \overline{\mathfrak{D}}$ 
pois, caso contrário, teríamos $\emptyset \in \mathfrak{D}$. Temos também que, dados $D, E \in \mathfrak{D}$, $\bar{D} \cap \bar{E}=\overline{D \cap E} \in \overline{\mathfrak{D}}$.

Demostramos agora que $\overline{\mathfrak{D}}$ é base uniforme. Sejam $x \in \prod^{\omega} X$ e $\bar{D} \in \overline{\mathfrak{D}}$. Como $\left(x_{i}, x_{i}\right) \in D$ para cada $i \in \omega$, então $(x, x) \in \bar{D}$, e assim $\overline{\mathfrak{D}}$ satisfaz a condição 2 .

Fixamos $\bar{D} \in \overline{\mathfrak{D}}$. Podemos escolher $B, C \in \mathfrak{D}$ tais que $B \circ B \subset D$ e $C^{-1} \subset D$. Assim, temos $\overline{B \circ B} \subset \bar{D}$ e $\overline{C-1} \subset \bar{D}$, e portanto $\overline{\mathfrak{D}}$ satisfaz as condições 3 e 4 .

O produto caixa uniforme caracteriza um intermediário entre o produto caixa e o produto de Tychonoff. Do mesmo modo que o produto caixa, a principal questão levantada pelo produto caixa uniforme diz respeito à sua paracompacidade. Bell estuda esta questão em [Bel14] e [Bel11]. 


\section{Referências Bibliográficas}

$\left[\mathrm{AST}^{+} 00\right]$ O. T. Alas, M. Sanchis, M. G. Tkačenko, V. V. Tkachuk e R. G. Wilson. Irresolvable and submaximal spaces: homogeneity versus $\sigma$-discreteness and new ZFC examples. Topology Appl., 107(3):259-273, 2000. 48

[Bel11] Jocelyn R. Bell. The uniform box product problem. ProQuest LLC, Ann Arbor, MI, 2011. Thesis (Ph.D.)-State University of New York at Buffalo. 90, 119, 122

[Bel14] Jocelyn R. Bell. The uniform box product. Proc. Amer. Math. Soc., 142(6):2161-2171, 2014. 90, 122

[Dia08] Rodrigo Roque Dias. Reflexão de funções cardinais e da metrizabilidade. Dissertação de Mestrado, IME-USP, 2008. 18

[Dow51] C.H. Dowker. On countably paracompact spaces. Can. J. Math., 3:219-224, 1951. 92

[Dow88] Alan Dow. An introduction to applications of elementary submodels to topology. Topology Proc., 13(1):17-72, 1988. 18

[DTTW02] A. Dow, M. G. Tkachenko, V. V. Tkachuk e R. G. Wilson. Topologies generated by discrete subspaces. Glas. Mat. Ser. III, $37(57)(1): 187-210,2002$. 3, 36, 38, 40

[Eng89] Ryszard Engelking. Genral Topology. Helderman Verlag, 1989. $10,20,31,33,75,117$

[Fer11] Gabriel Zanetti Nunes Fernandes. Teoria pcf e suas aplicações. Dissertação de Mestrado, IME-USP, 2011. 100

[FL91] James Foran e Paul Liebnitz. A characterization of almost resolvable spaces. Rend. Circ. Mat. Palermo (2), 40(1):136-141, 1991. 50 
[Har81] K. P. Hart. Strong collectionwise normality and M. E. Rudin's Dowker space. Proc. Amer. Math. Soc., 83(4):802-806, 1981. 93

[Har82] Klaas Pieter Hart. More on M. E. Rudin's Dowker space. Proc. Amer. Math. Soc., 86(3):508-510, 1982. 93

[Hec74] Stephen H. Hechler. On the existence of certain cofinal subsets of ${ }^{\omega} \omega$. Em Axiomatic set theory (Proc. Sympos. Pure Math., Vol. XIII, Part II, Univ. California, Los Angeles, Calif., 1967), páginas 155-173. Amer. Math. Soc., Providence, R.I., 1974. 71

[Hew43] Edwin Hewitt. A problem of set-theoretic topology. Duke Math. J., 10:309-333, 1943. 50

[HJ99] Karel Hrbacek e Thomas Jech. Introduction to set theory. 3rd, revised and expanded ed. New York, NY: Marcel Dekker, 1999. 10

[Hod84] Richard E. Hodel. Cardinal functions i. Em Handbook of SetTheoretic-Topology. North-Holland, Amsterdam, 1984. 15

[hs] Santi Spadaro (http://mathoverflow.net/users/11647/santi spadaro). A generalization of the arhangelskii theorem. MathOverflow. URL:http://mathoverflow.net/q/176221 (version: 2014-0720). 18

[Jec03] Thomas Jech. Set theory. Springer Monographs in Mathematics. Springer-Verlag, Berlin, 2003. The third millennium edition, revised and expanded. 6, 51, 71

[JMMP80] T. Jech, M. Magidor, W. Mitchell e K. Prikry. Precipitous ideals. J. Symbolic Logic, 45(1):1-8, 1980. 53

[Juh65] István Juhász. Untershuchungen über $\omega_{n} u$-metrisierbare räume. Ann. Uni. Sci Budapest, 1965. 103

[Kel75] J.L. Kelley. General Topology. Graduate Texts in Mathematics. Springer, 1975. 10, 20, 109, 117

[KS98] Menachem Kojman e Saharon Shelah. A ZFC Dowker space in $\aleph_{\omega+1}$ : an application of PCF theory to topology. Proc. Amer. Math. Soc., 126(8):2459-2465, 1998. 100 
[KST86] Kenneth Kunen, Andrzej Szymański e Franklin Tall. Baire irresolvable spaces and ideal theory. Ann. Math. Sil., (14):98-107, 1986. 53

[Kun78] Kenneth Kunnen. Paracompactness of box products of compact spaces. Transactions of American Mathematical Society, 240, Junho 1978. 17, 60, 62, 84

[Kun11] Kenneth Kunen. Set theory. London: College Publications, 2011. 6

[Law88] L. Brian Lawrence. The box product of countably many copies of the rationals is consistently paracompact. Trans. Amer. Math. Soc., 309(2):787-796, 1988. 76

[Lév02] A. Lévy. Basic Set Theory. Number v. 13 in Dover Books on Mathematics Series. Dover, 2002. 9

[Mic53] Ernest Michael. A note on paracompact spaces. Proc. Am. Math. Soc., 4:831-838, 1953. 21

[Mic56] Ernest Michael. Another note on paracompact spaces. Proceedings of the American Mathematical Society, 1956. 22

[Mic71] Ernest A. Michael. Paracompactness and the Lindelöf property in finite and countable Cartesian products. Compositio Math., 23:199-214, 1971. 102, 103

[Moo06] Justin Tatch Moore. A solution to the $L$ space problem. J. Amer. Math. Soc., 19(3):717-736 (electronic), 2006. 40

[Nyi75] Peter J. Nyikos. Some surprising base properties in topology. Em Studies in topology (Proc. Conf., Univ. North Carolina, Charlotte, N.C., 1974; dedicated to Math. Sect. Polish Acad. Sci.), páginas 427-450. Academic Press, New York, 1975. 100

[Per64] William J. Pervin. Foundations of General Topology. Academic Press, 1964. 111

[Pol74] R. Pol. Short proofs of two theorems on cardinality of topological spaces. Bull. Acad. Polon. Sci. Sér. Sci. Math. Astronom. Phys., 22:1245-1249, 1974. 16 
[Prz84] Teodor C. Przymusiński. Products of normal spaces. Em Handbook of set-theoretic topology, páginas 781-826. North-Holland, Amsterdam, 1984. 91

[Roi79] Judy Roitman. More paracompact box products. Proc. Amer. Math. Soc., 74(1):171-176, 1979. 72

[Roi07] Judith Roitman. Paracompactness of box products and their subspaces. Topology Proc., 31(1):265-281, 2007. 89

[Roi09] Judith Roitman. More paracompact subspaces of $\square(\omega+1)^{\omega}$. Topology Proc., 34:53-76, 2009. 89

[Roi11] Judith Roitman. Paracompactness and normality in box products: old and new. Babinkostova, L. (ed.) et al., Set theory and its applications. Annual Boise extravaganza in set theory, Boise, ID, USA, 1995-2010. Providence, RI: American Mathematical Society (AMS). Contemporary Mathematics 533, 157181 (2011)., 2011. 2, 23, 55, 74, 76, 89

[Rud55] Mary Ellen Rudin. Countable paracompactness and Souslin's problem. Canad. J. Math., 7:543-547, 1955. 92

[Rud71] Mary Ellen Rudin. A normal space $X$ for which $X \times I$ is not normal. Fund. Math., 1971. 4, 91, 92

[Rud72] Mary Ellen Rudin. The box product of countably many compact metric spaces. General Topology and Appl., 2:293-298, 1972. 2

[Rud74] Mary Ellen Rudin. Countable box products of ordinals. Trans. Amer. Math. Soc., 192:121-128, 1974. 84

[Rud75] Mary Ellen Rudin. Lectures on set theoretic topology, chapter X, páginas iv +76 . Published for the Conference Board of the Mathematical Sciences by the American Mathematical Society, Providence, R.I., 1975. Expository lectures from the CBMS Regional Conference held at the University of Wyoming, Laramie, Wyo., August 12-16, 1974, Conference Board of the Mathematical Sciences Regional Conference Series in Mathematics, No. 23. 32, 35

[Rud84] Mary Ellen Rudin. Dowker spaces. Em Handbook of SetTheoretic-Topology. North-Holland, Amsterdam, 1984. 91 
[RW90] Judith Roitman e Scott Williams. The paracompactness of spaces related to uncountable box products. Topology Proc., 15:135141, 1990. 86

[ST71] R. M. Solovay e S. Tennenbaum. Iterated Cohen extensions and Souslin's problem. Ann. of Math. (2), 94:201-245, 1971. 92

[Tka11] V.V. Tkachuk. A Cp-Theory Problem Book: Topological and Function Spaces. Problem Books in Mathematics. Springer, 2011. 42

[TV02] A. Tamariz-Mascarúa e H. Villegas-Rodríguez. Spaces of continuous functions, box products and almost- $\omega$-resolvable spaces. Commentat. Math. Univ. Carol., 43(4):687-705, 2002. 3, 43

[TW12] V. V. Tkachuk e R. G. Wilson. Box products are often discretely generated. Topology Appl., 159(1):272-278, 2012. 40

[Vau72] J. E. Vaughan. Linearly stratifiable spaces. Pacific J. Math., 43:253-266, 1972. 101

[Vau75] J. E. Vaughan. Nonnormal products of $\omega_{\mu}$-metrizable spaces. Proc. Amer. Math. Soc., 51:203-208, 1975. 4, 91, 100

[vD75] Eric K. van Douwen. The box product of countably many metrizable spaces need not be normal. Fund. Math., 88(2):127-132, 1975. 104

[vD80] Eric K. van Douwen. Covering and separation properties of box products. Em Surveys in general topology, páginas 55-129. Academic Press, New York, 1980. 2, 29, 55, 74, 87, 89

[vD93] Eric K. van Douwen. Applications of maximal topologies. Topology Appl., 51(2):125-139, 1993. 38

[Wil77a] Scott W. Williams. Boxes of compact ordinals. Em Proceedings of the 1977 Topology Conference (Louisiana State Univ., Baton Rouge, La., 1977), II, volume 2, páginas 631-642 (1978), 1977. 84

[Wil77b] Scott W. Williams. Is ${ }^{\omega}(\omega+1)$ paracompact? Em Topology Proceedings, Vol. I (Conf., Auburn Univ., Auburn, Ala., 1976), páginas 141-146. Math. Dept., Auburn Univ., Auburn, Ala., 1977. 84,86 
[Wil84] Scott W. Williams. Box products. Em Handbook of Set-Theoretic Topology. North-Holland, Amsterdam, 1984. 2, 23, 32, 55, 72, $74,90,115$

[Win94] Louis Wingers. Box products of $\sigma$-compact spaces. Topology Appl., 56(2):185-197, 1994. 78, 82

[YW87] Soulian Yang e Scott W. Williams. On the countable box product of compact ordinals. Em Proceedings of the 1987 Topology Conference (Birmingham, AL, 1987), volume 12, páginas 159-171, 1987. 84 


\section{Índice Remissivo}

$1^{o}$ axioma da enumerabilidade, 16

$2^{\circ}$ axioma da enumerabilidade, 15

$3^{\circ}$ axioma da enumerabilidade, 15

$={ }^{*}$-transversal, 76

$C(X), 42$

$C(X, Y), C(X), 41$

$C_{p}(X), 42$

$F_{\sigma}, 10$

$G_{\delta}, 10,18$

$G_{\delta}$-modificação, 65

$P_{\kappa}$-espaço, 22

$T_{0}, 11$

$T_{1}, 11$

$T_{2}, 11$

$T_{3}, 11$

$T_{4}, 12$

$T_{3 \frac{1}{2}}, 12$

$C_{\square}(X), 42$

$C_{\square}$-discreto, 43

$\kappa$-Lindelöf, 21

$\kappa$-Produto Caixa, 26

$\kappa$-caixa aberta, 27

$\kappa$-metrizável, 24, 115

$\kappa$-resolúvel, 43

$\lambda$-escala, 70

$\sigma$-discreto, 48

árvore, 9

altura, 9

base de filtro, 6

caixa aberta, 26 caixa fechada, 26

caráter, 16

caráter de dispersão, 48

cardinal, 8

cardinal mensurável, 51

cardinal regular, 10

cardinal singular, 10

celularidade, 15

cofinalidade, 10

coletivamente normal, 13

completamente regular, 12

conjunto cofinal, 10

densidade, 15

denso-em-si-mesmo, 37, 45

discretamente gerado, 36

disperso, 13

enumeravelmente paracompacto, 92

Espaço de Dowker, 91

espaço de Tychonoff, 12

espaço maximal, 37

espaço submaximal, 37

família afunilada, 79

família celular, 15

família discreta, 13

Família dominante, 70

Família ilimitada, 70

Família localmente finita, 20

família simples, 79

filtro, 6 
filtro $\kappa$-completo, 7

filtro livre, 7

filtro principal, 7

fortemente disjuntas, 79

função cardinal, 15

função uniformemente contínua, 108

grau de Lindelöf, 15

Hausdorff, 11

Hipótese da Ordem, 76

monotonamente normal, 13

Nabla Lema, 115

Nabla Lemma, 60

normal, 12

ordem, 8

ordinal, 8

P-espaço, 12

paracompacto, 20

peso, 15

primeiro enumerável, 16

Produto Caixa, 26

produto caixa de uniformidades, 120 produto caixa uniforme, 121

Produto de Tychonoff, 26

produto de Tychonoff de uniformidades, 119

produto nabla, 56

quase resolúvel, 44

quase- $\omega$-resolúvel, 44

rank, 14

regular, 11

resolúvel, 43

segundo enumerável, 15

separável, 15

sequência de Cantor-Bendixon, 14

soma topológica, 45 spt, 26

suporte, 26

ultrafiltro, 6

ultraparacompacto, 20

uniformizável, 110

zero dimensional, 12 